Review

\title{
Critical Review of Intelligent Battery Systems: Challenges, Implementation, and Potential for Electric Vehicles
}

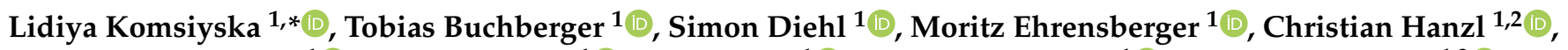

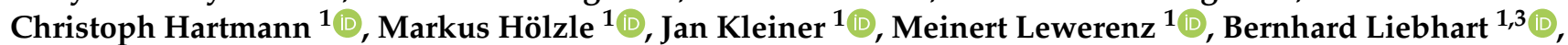

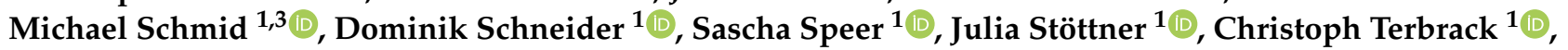 \\ Michael Hinterberger ${ }^{2}$ and Christian Endisch ${ }^{1,3}$
}

1 Institute of Innovative Mobility, TH Ingolstadt, 85049 Ingolstadt, Germany; t.buchberger@mytum.de (T.B.); simon.diehl@thi.de (S.D.); moritz.ehrensberger@tum.de (M.E.); christian.hanzl@thi.de (C.H.); christoph.hartmann@thi.de (C.H.); markus.hoelzle@thi.de (M.H.); jan.kleiner@thi.de (J.K.); meinert.lewerenz@thi.de (M.L.); bernhard.liebhart@thi.de (B.L.); michael.schmid@thi.de (M.S.); dominik.schneider@thi.de (D.S.); sascha.speer@thi.de (S.S.); julia.stoettner@thi.de (J.S.); christoph.terbrack@thi.de (C.T.); christian.endisch@thi.de (C.E.)

2 Development High Voltage Battery Systems, AUDI AG, 85045 Ingolstadt, Germany; michael.hinterberger@audi.de

3 Chair of Electrical Drive Systems and Power Electronics, TU Munich, 80333 Munich, Germany

* Correspondence: els@thi.de or lidiya.komsiyska@thi.de

\section{check for}

updates

Citation: Komsiyska, L.; Buchberger, T.; Diehl, S.; Ehrensberger, M.; Hanzl,

C.; Hartmann, C.; Hölzle, M.; Kleiner,

J.; Lewerenz, M.; Liebhart, B.; et al.

Critical Review of Intelligent Battery Systems: Challenges, Implementation, and Potential for Electric Vehicles. Energies 2021, 14, 5989. https:// doi.org/10.3390/en14185989

Academic Editor: Carlos Miguel Costa

Received: 27 July 2021

Accepted: 9 September 2021

Published: 21 September 2021

Publisher's Note: MDPI stays neutral with regard to jurisdictional claims in published maps and institutional affiliations.

Copyright: (c) 2021 by the authors. Licensee MDPI, Basel, Switzerland. This article is an open access article distributed under the terms and conditions of the Creative Commons Attribution (CC BY) license (https:// creativecommons.org/licenses/by/ $4.0 /)$.
Abstract: This review provides an overview of new strategies to address the current challenges of automotive battery systems: Intelligent Battery Systems. They have the potential to make battery systems more performant and future-proof for coming generations of electric vehicles. The essential features of Intelligent Battery Systems are the accurate and robust determination of cell individual states and the ability to control the current of each cell by reconfiguration. They enable high-level functions like fault diagnostics, multi-objective balancing strategies, multilevel inverters, and hybrid energy storage systems. State of the art and recent advances in these topics are compiled and critically discussed in this article. A comprising, critical discussion of the implementation aspects of Intelligent Battery Systems complements the review. We touch on sensing, battery topologies and management, switching elements, communication architecture, and impact on the single-cell. This review contributes to transferring the best technologies from research to product development.

Keywords: smart battery; intelligent battery system; advanced monitoring; reconfigurable battery; battery management system; electric vehicle

\section{Introduction}

Alternative drive technologies, such as Battery Electric Vehicles (BEVs), have come into focus for a variety of reasons. As a consequence, intensive global research and engineering development is currently conducted in order to boost the electromobility. A key element of the BEV drive train is the energy storage, commonly realized by a rechargeable battery system. As for the most promising storage technologies, lithium-ion (Li-ion) battery systems have been used almost exclusively in electric vehicles in recent years. The reason is that Li-ion batteries exhibit high power and energy density, long cycle-life, and low self-discharge when compared to other common battery technologies [1].

However, there are still drawbacks associated with this technology namely a short range, long charging times, high cost, and issues with safety and reliability. Multiple cells need to be connected in parallel and in series to fulfill performance requirements, as the individual cell's power and capacity is limited. As shown by recent studies, the interconnection of many cells leads to inhomogeneities on system-level due to cell-to-cell variation [2-6]. Parameter inconsistencies originate from slight differences during the production processes. Variations even exist between identical, brand-new cells from the 
same batch [4] and increase during use [3]. This fact, in combination with a fixed multicell configuration, results in the weakest cell limiting the entire battery system [6]. To overcome these drawbacks, Reconfigurable Battery Systems (RBSs) incorporate switches which allow individual cells to be temporarily excluded from the current path. In addition, the reconfiguration offers a great potential for continuous variation of the batteries' voltage level, which results in Multilevel Inverters (MLIs). Controlling the current path by switches eventually enables the combination of different types of storage devices in Hybrid Battery Storage Systems (HBSSs).

Reconfiguration alone does not cancel the limitations of conventional battery systems if any cell's state is unknown. Without this knowledge, reasonable operation of the switches is not possible in practice. However, in common battery systems, the states of each cell are rarely available due to multiple reasons. On the one hand, a large number of sensors and cabling efforts would be necessary; on the other hand, many relevant cell states such as the State of Charge (SOC) or the State of Health ( $\mathrm{SOH}$ ) cannot be measured directly. Thus, a significant effort has to be made in terms of monitoring the state of each battery cell.

The numerous challenges that arise from non-reconfigurable battery systems with unknown states of the individual cells are addressed by researchers and studies in various fields. This review comprises the topics depicted in Figure 1. The challenge of advanced monitoring is addressed in the literature by two complementary approaches. Firstly, model-based or datadriven fault diagnosis, state, and parameter estimation is achieved by applying algorithms. Secondly, the implementation of considerably complex sensors is mandatory for reliable data acquisition. Focusing on flexible configurations to avoid the limits of non-reconfigurable multi-cell battery systems, possible functionalities are displayed, whereby the necessary system topologies are discussed on micro and macro levels.

\begin{tabular}{l}
2 Algorithms \\
2.1 Fault Diagnosis \\
2.2 Parameter Estimation \\
3 Sensor Implementation \\
2.3 Core Temperature \\
3.1 Current \\
3.6 State of Charge of Health \\
3.2 Voltage \\
3.3 Temperature \\
3.4 Impedance \\
3.5 Mechanical \\
\hline
\end{tabular}
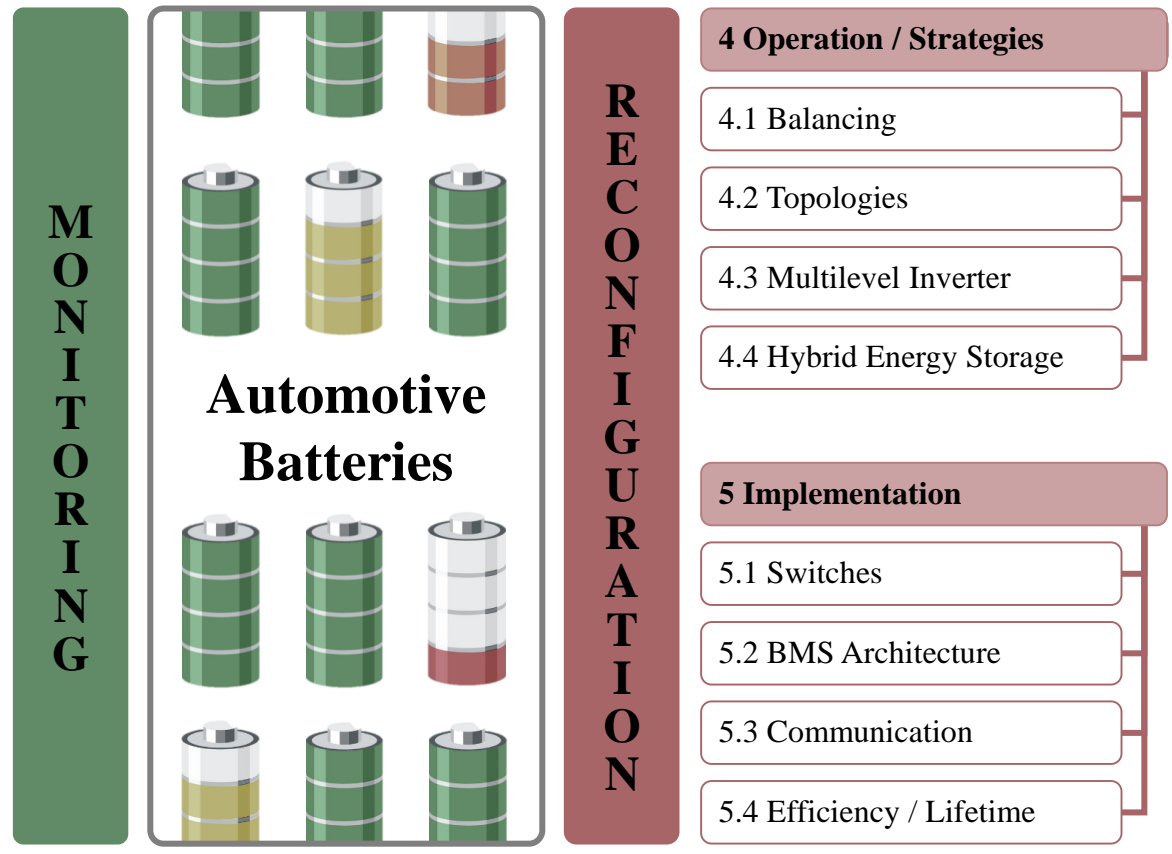

Figure 1. Overview of the aspects of advanced monitoring and reconfiguration to solve the main challenges of automotive batteries. The figure also serves as a graphical table of contents.

As a main aspect of this review, the extensive literature on advanced monitoring and reconfiguration in Sections 2 and 4 is not only methodically reviewed and sorted, but also selected and evaluated with regard to its application in electric vehicles. Thus, the focus is on approaches that are not only applicable on laboratory level but also in a BEV. Furthermore, general state estimation approaches are filtered to meet the challenges of the automotive application such as single cell state estimation in a system. 
As a special aspect, the review additionally provides insights into application and applicability by discussing aspects of the implementation of sensor technology and reconfiguration in Sections 3 and 5. This is important since the application of the advanced functionalities may require specific hardware but in any case an improved conceptualization on a system level. This is depicted in Figure 2 where schematically the switches for reconfiguration as well as possible sensor implementation and the necessary communication or Battery Management System (BMS) integration is shown. First, concepts combining several of the above functions with corresponding hardware and system approaches can be found in the literature presented as "intelligent" or "smart" batteries [7-10]. In addition, in the multitude of patents that arise in this field, different aspects are in the focus addressing smart or intelligent battery devices: Intel focuses on integrated sensing [11], Bosch is patenting a device for regulating the system voltage adaptively [12] and Audi outlines an integral smart battery cell with sensing, communication, and integrated reconfiguration functionality $[13,14]$. On the research side, Saidani et al. [7] present a battery system, where each battery cell is customized with voltage and temperature sensors, a digital processing unit, and data storage. Their smart system allows battery monitoring on a cell level. Schneider et al. [8] use an intelligent battery with a cell individual current and temperature sensor and reconfiguration functionality for an improved online parameter estimation. Lorentz et al. [9] focus on contactless data transmission to realize smart battery cell monitoring, where each cell is equipped with its own monitoring circuit board, and all boards exchange information among each other. In contrast, Kim et al. [10] denominate their battery system as smart battery, where the ordinary battery system is augmented by a switching circuit board which allows activation and bypassing of each cell. Finally, an overview of the latest progress of research regarding intelligent battery systems is given by Wei et al. [15].

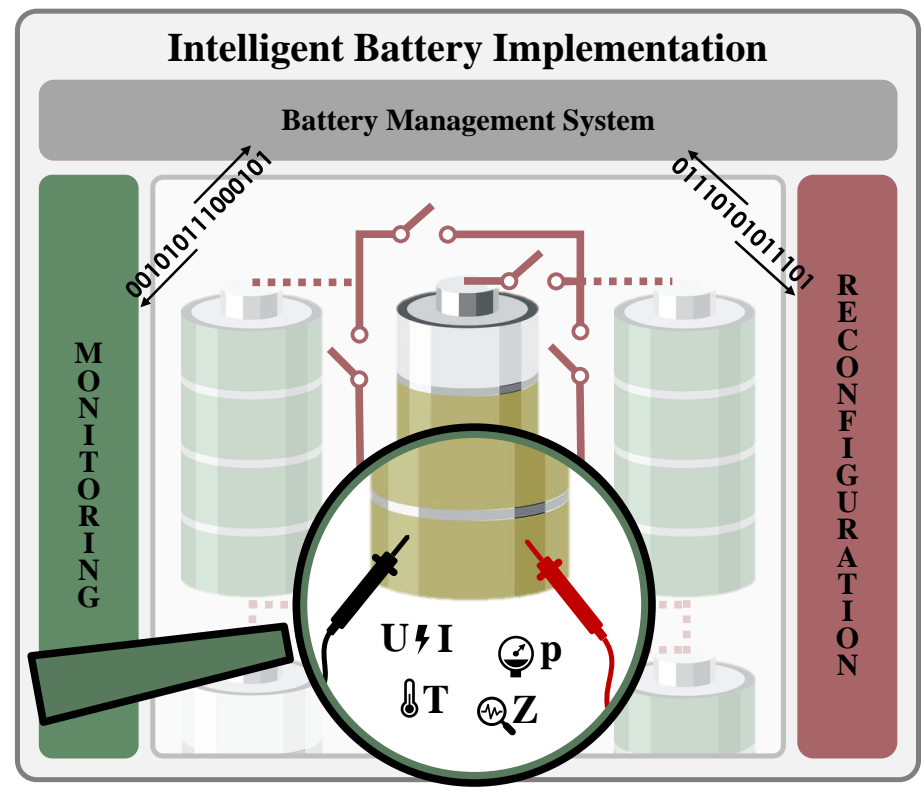

Figure 2. Schematic visualization of the aspects related to the implementation of intelligent battery systems with reconfiguration and advanced monitoring functionality.

There are review articles in the fields of BMSs [1,16-18], RBSs [19], MLIs [20], smart sensing [15,21-23], fault diagnostics [24], and thermal management [16]. This review is the first to provide an overview that holistically connects the novel functions with a focus on the application in electric vehicles. The used tools and methods originate from the broad field of artificial intelligence. Thus, in this work, the literature is summarized under the subject of Intelligent Battery Systems (IBSs). IBSs as a new technological advance represent a promising but also a challenging approach to significantly improve the reliability, safety, 
and efficiency of BEVs. In the light of the variety of approaches, we specify those battery systems as intelligent that incorporate:

- additional sensors or advanced monitoring functions on cell level and/or

- actuators such as switches to modify the system's topology reversibly.

Not only the hardware itself but the application of algorithms and methods from the field of machine learning are necessary for a battery system to be stated as intelligent.

Addressing the related topics, this review is organized as follows: In Section 2, the latest developments in advanced monitoring methods for IBSs are discussed including fault diagnosis, online parameter estimation as well as the determination of temperature, SOC, $\mathrm{SOH}$, and State of Function (SOF). The related sensor technologies and their corresponding implementation are discussed in Section 3. Subsequently, Section 4 deals with the broad field of reconfiguration of battery systems enhanced by actuators and corresponding operation strategies on a system level. This includes simple serial/parallel reconfiguration as well as advanced approaches such as hybrid energy storage concepts and Multilevel Inverters. The focus of Section 5 is on practical issues when applying reconfiguration functionality in IBSs. As will become apparent during the course of this review, the actual implementation of IBS poses some challenges which are discussed in this section. Finally, the main results are summarized and an outlook to further developments is given.

\section{Advanced Battery Monitoring}

Commercially available automotive battery systems normally feature cell voltage monitoring, to prevent single cells from undervoltage or overvoltage, and a current sensor on the system level. Furthermore, the battery pack's temperature is monitored at a few measurement points within the battery system. Further monitoring of the individual cells is usually avoided due to additional costs and cabling effort or limited computing resources in the Battery Management System (BMS) for the online determination of non-measurable cell states. At the same time, there are many approaches in research to determine the highly relevant behavior of the individual cell during operation more precisely. While discussing the different aspects of fault diagnosis, parameter estimation, and state determination, it is assumed that the required data are available. The integration of sensors and measuring methods is discussed afterwards, in Section 3.

In terms of advanced monitoring functionalities, information from additional sensors is not necessarily required in all cases. Collecting and linking sensor data of the whole battery system, approaches are enabled that make use of the distributed information and its correlation. An example for a promising application is sensor fault detection and localization. However, only approaches feasible for online application in Intelligent Battery Systems (IBSs) are considered. For the application in Battery Electric Vehicles (BEVs), it is mandatory that the algorithms are at least real-time capable on limited hardware resources and viable with ordinary sensors that are expected to be used on a cell level within an IBS.

\subsection{Fault Diagnosis}

To ensure that each single cell of a battery system is within its safe operating range, a basic function of BMS is to maintain lower and upper voltage, current, and temperature limits. In addition, more advanced fault diagnosis is desired to detect faults that cannot be avoided by the mere assertion of limits, at an early stage in order to prevent serious consequences. Fault diagnosis for battery systems has therefore become a discipline of its own in recent years. In the literature, a distinction is made between several levels:

- fault detection: recognition of faults;

- fault isolation: localization of faults;

- fault identification: estimation of the type and amplitude of faults;

- fault tolerance: (limited) continuation of operation in the presence of diagnosed faults.

Fault diagnosis usually means the detection and isolation of a fault. 
Faults can be classified into internal and external faults $[25,26]$. The former designate cell defects such as overcharging, over-discharging, External Short Circuits (ESCs)/Internal Short Circuits (ISCs), overheating, accelerated aging, or thermal runaway. An ISC is caused by a defect in the separator layer between the electrodes [27]. If the short circuit does not occur between the electrodes but at the tabs, it is called an ESC [24,25]. Short-circuits contribute to the inhomogeneity in the battery system and can lead to deep-discharge. Especially low resistances lead to a fast self-discharge of the affected cells and to an increased heat generation, which can lead to overheating. Overheating is also caused by an external heat input or a defect in the cooling system. In general, increased temperature leads to accelerated degradation [28], which is characterized by excessive capacity fading and resistance increase. Any of the internal faults can result in thermal runaway which is also the most catastrophic fault and is associated with battery swelling, electrolyte leakage, smoke, fire, and explosion $[25,26]$.

External faults are sensor or actuator faults in the battery system [26]. Currently, conventional voltage, current, and temperature sensors are used. Defective sensors can lead to an incorrect state estimation, which in turn leads to faulty operation, such as overcharging, under-discharge or over-heating, and accelerated degradation of the battery system [29]. Actuators include the cell and high voltage connectors as well as the cooling system. Similar to the sensors, the connections of the power path can be faulty or degrade over time due to vibration or corrosion [30]. The defective cell connection leads to increased resistance, which in parallel-connected cells causes unequal current distribution and in serial-connected battery systems local hotspots [31]. A failure in the cooling system is provoked by a leak in the cooling channels or a faulty fan [16]. In general, external faults can raise further internal faults or thermal runaway of the cells if they are not detected or not responded to appropriately.

Tables 1 and 2 provide an overview of the literature on fault diagnosis. There are a variety of methods for diagnosing internal and external faults (reported e.g., by [1,17,18,25,26,29]). The methods for fault diagnosis can be classified into model-based, knowledge-based, and data-driven approaches [1]. In battery systems, the most common approach for fault diagnosis are model-based methods. They rely on state or parameter estimation, parity space, or structural analysis. For state estimation, the system is reconstructed with filters or observers. A comparison of the model with the measured data of the real system provides a residual which is used for fault diagnosis [32]. The algorithms are based on Particle Filter (PF), Kalman Filter (KF), or a state observer [25] (see differentiation in Tables 1 and 2). With parameter estimation, model or fault parameters are estimated using the measured data. If a model or fault parameter leaves the permitted range, a fault is detected. It is therefore easier to isolate faults with parameter estimation than with state estimation [26]. Parity space based methods are used to verify the relationship between the input and output variables of a system [33]. The analysis of the resulting residuals is used for fault diagnosis. Structural analysis is a tool for identifying the overdetermined part of a system. Exploiting redundancy in the system enables the generation of residuals for fault diagnosis [34].

Knowledge-based methods do not require a mathematical or analytical model and are particularly suitable for nonlinear and complex systems such as lithium-ion (Li-ion) battery systems [26]. For fault diagnosis, observations and knowledge about battery systems are used, which was previously gained from studies on fault mechanisms. The approaches are based on graph theory, expert systems, or fuzzy logic [25].

Data-driven methods directly analyze the measured data of the battery system. While some methods do not require a model at all and just extract features from measured data or use a special sensor topology [35-37], other methods learn a data model [38,39]. The algorithms used are based on signal processing, Artificial Neural Networks (ANNs), and support vector machines [26]. Signal processing methods use the wavelet transform, Shannon entropy, or the correlation of the signals. ANNs are able to learn during operation and have the potential to be computationally efficient but have a poor generalization capability. While the generalization capability of support vector machines is significantly 
better, the required computational effort for large data sets increases rapidly, since the data are transformed into a high-dimensional space by a kernel function before classification.

Since data-driven methods, such as [40-42], are often prone to cell inconsistencies, model-based methods are most promising in IBSs. However, for the model-based methods, it is crucial that they are computationally efficient. The methods presented in [43-45] are expected to have high computational loads in practical applications, making them challenging to use at the system level. The switches in IBSs open up the possibility for active fault diagnosis, as shown in [32], by selectively actuating the switches. The active fault diagnosis is a promising field in which further research is required in the future.

Due to the larger number of components, the probability of failure of a component rises in IBSs [34]. For example, the number of sensors increases significantly due to the single-cell sensors compared to conventional battery systems. However, the additional sensors allow for a better detectability and isolation of faults. The large number of sensors on system level also allows a plausibility check of sensor values and a reconstruction of the correct values in case of a fault. Furthermore, an IBS allows fault diagnosis at a cell level. This design is robust against faults that occur due to signal transmission to the central or neighboring cell BMS. Basic functions such as maintaining voltage, current, and temperature limits are possible with limited computing power. Even a hard ESC can be detected at cell level, e.g., by means of the current increase. In contrast, advanced functions such as the detection of ISCs or accelerated aging are more promising on the overall BMS. These sometimes require more computing power [44] but benefit from inter-cell comparison [38,39]. 
Table 1. Overview of the literature related to fault diagnosis of external faults.

\begin{tabular}{|c|c|c|c|c|c|c|}
\hline & & \multicolumn{3}{|c|}{ Sensor Fault } & \multicolumn{2}{|c|}{ Actuator Fault } \\
\hline & & Voltage & Current & Temperature & Connection & Cooling System \\
\hline \multirow{8}{*}{ 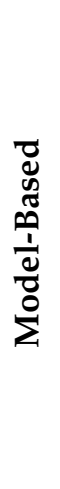 } & Particle Filter & {$[46,47]$} & {$[46,47]$} & [46] & & \\
\hline & Kalman Filter & {$[32,48-53]$} & {$[32,48-53]$} & {$[32,51]$} & {$[32,54]$} & \\
\hline & Luenberger Observer & & & & & [55] \\
\hline & Lyapunov-Analysis Nonlinear Observer & & & & & [56] \\
\hline & Proportional Integral Observer & & {$[58]$} & & & \\
\hline & Sliding Mode Observer & [59] & [59] & [59] & [54] & \\
\hline & Recursive Least Squares & {$[25,46,49]$} & {$[25,46,49]$} & {$[46]$} & {$[60]$} & \\
\hline & Parity space: Nonlinear Parity Equations & {$[33,61]$} & {$[33,61]$} & {$[33,61]$} & & [61] \\
\hline \multirow{9}{*}{ مُ } & Structural Analysis & {$[32,34,51,62,63]$} & {$[32,34,51,62,63]$} & {$[32,34,51,62,63]$} & {$[32,34]$} & {$[62,63]$} \\
\hline & Wavelet Transform & & & & & \\
\hline & Shannon Entropy/Mutual Information & & & & {$[30,64-67]$} & \\
\hline & Sensor Topology & {$[35-37]$} & & & [37] & \\
\hline & Incremental Capacity Analysis & & & & & \\
\hline & CCCV Transform & & & & & \\
\hline & Clustering & & & & {$[68]$} & \\
\hline & Anomaly Detection & {$[38]$} & {$[38]$} & & {$[38]$} & \\
\hline & Neural Network & & & & & \\
\hline
\end{tabular}


Table 2. Overview of literature related to fault diagnosis of internal faults

\begin{tabular}{|c|c|c|c|c|c|c|c|c|c|c|c|}
\hline & & \multirow[b]{2}{*}{ 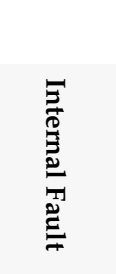 } & \multirow[b]{2}{*}{ 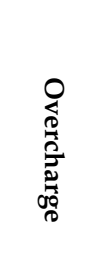 } & \multirow[b]{2}{*}{ 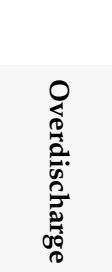 } & \multicolumn{3}{|c|}{ Short Circuit } & \multirow[b]{2}{*}{ 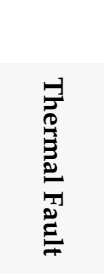 } & \multicolumn{3}{|c|}{ Accelerated Degradation } \\
\hline & & & & & $\begin{array}{l}n \\
0 \\
\vdots \\
0 \\
\vdots \\
\vdots \\
\vdots\end{array}$ & $\vec{n}$ & 罢 & & 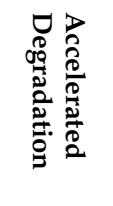 & 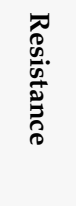 & 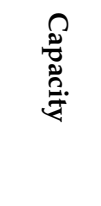 \\
\hline \multirow{9}{*}{ 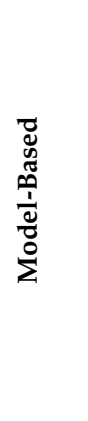 } & Particle Filter & & & & & & & & [45] & & \\
\hline & Luenberger Observer & & & & & & & [55] & & [72] & \\
\hline & Lyapunov-Analysis Nonlinear Observer & & & & & & & [56] & & & \\
\hline & Partial-Differential-Equation Observer & & & & & & & [57] & & & \\
\hline & Proportional Integral Observer & & & & [73] & [74] & & & & & \\
\hline & Sliding Mode Observer & & & & & & & & & & \\
\hline & Recursive Least Squares & & & & & {$[70,71,75-78]$} & & & & & [60] \\
\hline & Parity Space: Nonlinear Parity Equations & & & & & & & & & & \\
\hline & Structural Analysis & & & & & {$[32,34]$} & {$[32,34]$} & & & & \\
\hline \multirow{7}{*}{ 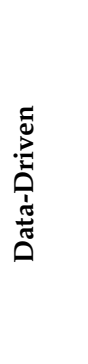 } & Correlation Coefficient & [81] & & & {$[81,82]$} & {$[37,69,81,82]$} & {$[37,81,82]$} & & & & \\
\hline & Shannon Entropy/Mutual Information & {$[83,84]$} & [64] & [64] & {$[64]$} & [85] & & [86] & & & \\
\hline & Sensor Topology & {$[35,36]$} & & & & [37] & [37] & & & & \\
\hline & Incremental Capacity Analysis & & & & & & & & & & [40-42] \\
\hline & CCCV Transform & & & & & [79] & & & & & \\
\hline & Clustering & & [87] & [87] & [87] & & & [87] & {$[88,89]$} & & \\
\hline & Anomaly Detection & & [87] & [87] & {$[38,87]$} & [39] & & {$[87,90]$} & {$[88,89]$} & & \\
\hline \multirow{5}{*}{ 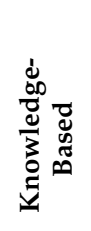 } & Artificial Neural Network & & & & & & & [91] & & & \\
\hline & Rule-Based & & & [92] & & [93] & [94] & & & & \\
\hline & Fuzzy Logic & & {$[95,96]$} & {$[95,96]$} & & & & & [95] & & \\
\hline & Random Forests Classifier & & & & & & [97] & & & & \\
\hline & Artificial Neural Network & [98] & & & & & & & & & \\
\hline
\end{tabular}




\subsection{Online Identification of Model Parameters}

In the field of battery monitoring, Equivalent Circuit Models (ECMs) are popular to model the battery's electrical behavior. Thereby, the level of detail ranges from simple Rint models up to more complex N-RC Thevenin models (see Figure 3). The necessary parameters are basically determined by experimental tests such as Hybrid Pulse Power Characterization (HPPC) and corresponding fitting procedures. For in-use model operation, however, varying parameters must be considered due to temperature, aging, or other influences. As a consequence, the adaptation and online identification of model parameters is mandatory for the use in IBSs. Online identification is primarily about shifting temperature and State of Charge (SOC) dependencies as well as the change of absolute parameter values due to aging. With current and voltage sensors on the cell level, various approaches that have been studied in laboratory settings in recent years are transferable to IBSs.

(d) N RC Thevenin model

(c) Dual Polarization model

(b) Thevenin model

(a) Rint model

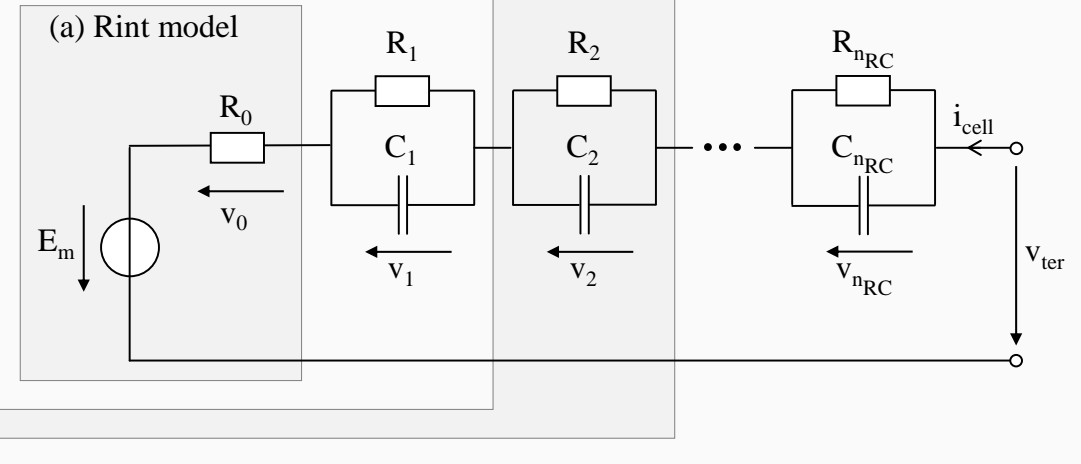

(e) RC model

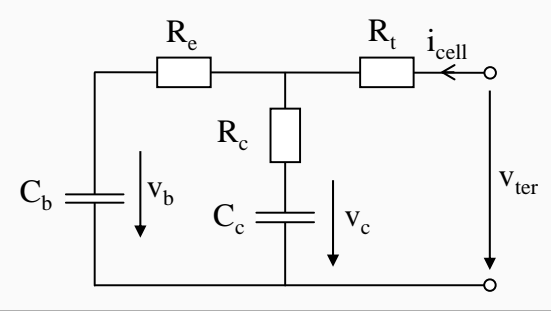

(f) PNGV model

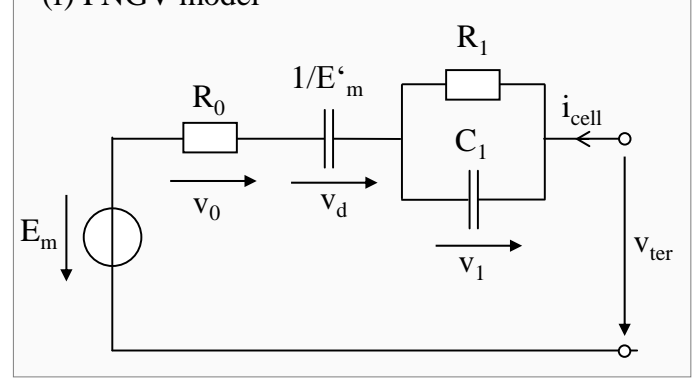

Figure 3. Typical equivalent electrical circuit models used for vehicle batteries: (a) Rint model; (b) Thevenin model; (c) Dual Polarization model; (d) N RC Thevenin model; (e) RC model; and (f) PNGV model. From [99] with permission from Elsevier, Copyright 2020.

Generally, two representations of the underlying ECM are distinguished. Firstly, the state space representation in discrete form (see Equations (1) and (2)) is beneficial for KF approaches like the Extended Kalman Filter (EKF) [100]. Parameter estimation is commonly combined with SOC estimation when applying a Dual Kalman Filter (DKF) [101-104]. In the following equation, $\Delta T$ is the discrete simulation step size, $\tau_{i}$ is the time constant of the $i$-th RC element with resistance $R_{i}$, whereas $R_{0}$ expresses the ohmic resistance. $Q$ is the cell capacity and $\eta$ its coulombic efficiency, which is nearly 1 . The excitation current represents the input $u$, whereas the terminal voltage is measured as $u$. Finally, the dependence of Open Circuit Voltage (OCV) from SOC is depicted by the function $u_{\mathrm{OC}}(S O C)$. The weak influence of $Q$ on the measurement impedes its estimation. 


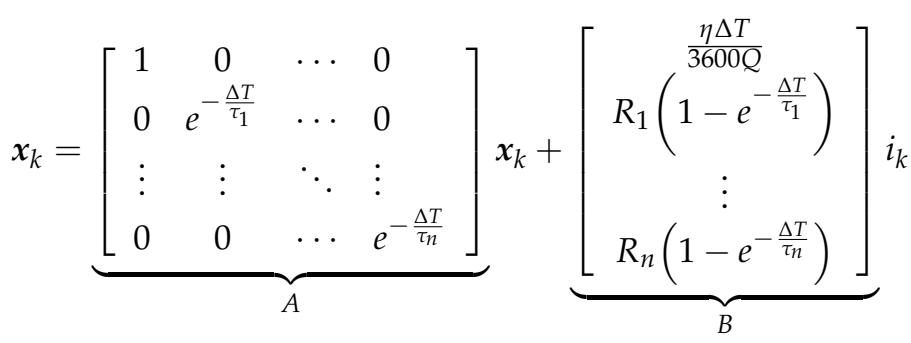

$$
\begin{aligned}
& u_{k}=\underbrace{\left[\begin{array}{llll}
\frac{u_{\mathrm{OC}}\left(S O C_{k}\right)}{S O C_{k}} & 1 & \cdots & 1
\end{array}\right]}_{C} \boldsymbol{x}_{k}+\underbrace{R_{0}}_{D} i_{k}
\end{aligned}
$$

Secondly, based on the transfer function of the battery model, a linear regression model (see Equations (3) and (4)) is often formed and Recursive Least Squares (RLS) algorithms are applied [102,105-107].

$$
u_{k}=\underbrace{\left[\begin{array}{llllll}
1 & u_{k-1} & u_{k-2} & i_{k} & i_{k-1} & i_{k-2}
\end{array}\right]}_{\boldsymbol{\Phi}_{k}^{T}} \cdot \underbrace{\left[\begin{array}{c}
\frac{1-a_{1}}{u_{\mathrm{OC}}} \\
a_{1} \\
a_{2} \\
a_{3} \\
a_{4} \\
a_{5}
\end{array}\right]}_{\boldsymbol{\Theta}_{k}}
$$

with

$$
\begin{aligned}
R_{0} & =\frac{a_{3}-a_{4}+a_{5}}{1+a_{1}-a_{2}} \\
R_{0}+R_{1}+R_{2} & =\frac{a_{3}+a_{4}+a_{5}}{1-a_{1}-a_{2}} \\
\tau_{1} \tau_{2} & =\frac{\Delta T^{2}\left(1+a_{1}-a_{2}\right)}{4\left(1-a_{1}-a_{2}\right)} \\
\tau_{1}+\tau_{2} & =\frac{\Delta T\left(1+a_{2}\right)}{1-a_{1}-a_{2}} \\
R_{0}\left(\tau_{1}+\tau_{2}\right)+R_{1} \tau_{2}+R_{2} \tau_{1} & =\frac{\Delta T\left(a_{3}-a_{5}\right)}{1-a_{1}-a_{2}}
\end{aligned}
$$

Usually, the input noise is neglected, which leads to biased estimates. However, in [108], a Weighted Total Least Squares (WTLS) algorithm is used to take input noise into account. Further approaches based on the regression model for parameter identification are observers [109], Lagrange multipliers [110], and the Genetic Algorithm (GA) [111]. The latter is hardly implementable on-board due to the high computational cost associated with it. An overview of identification methods and the corresponding literature is depicted in Figure 4. While in most cases voltage and current are used for time domain parameter identification, in [112], the impedance is determined in the frequency domain and an ECM is parameterized based on the results.

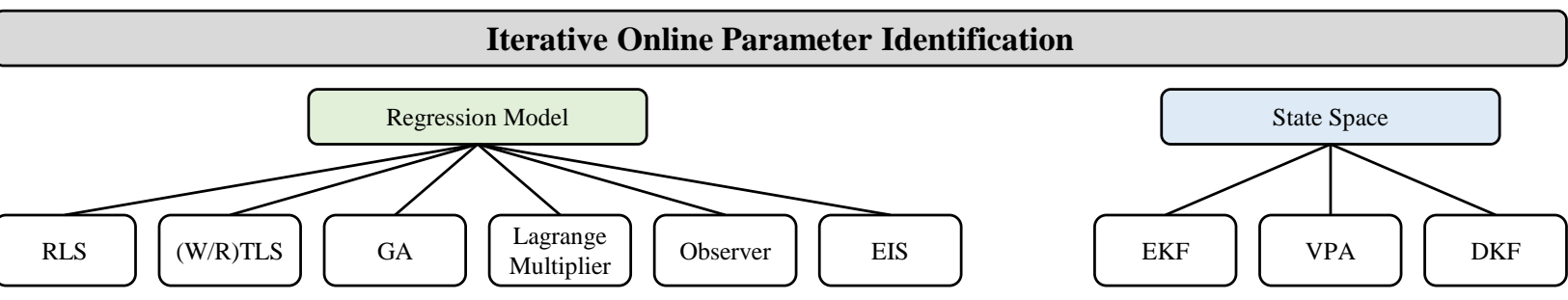

Figure 4. Overview of modeling and online parameter identification methods and the corresponding literature. 
The literature published in recent years in terms of battery parameter estimation is manifold. However, with respect to IBSs, efficient and robust algorithms are needed to be applied on-board. Cell individual parameterization is enabled by sensing capabilities of cell current and voltage, but the resulting computational burden has to be considered carefully.

\subsection{Online Identification of Core Temperature}

The temperatures of most interest in a Li-ion battery system are the cell's individual core temperatures which mainly influence aging and safety. Furthermore, the individual electrical cell parameters are strongly temperature dependent and dominated by the region with the highest temperature [113]. However, large format cells often used in automotive applications are facing the challenge of temperature gradients inside the cells and the core temperature can significantly differ from the temperature value measured by the external sensors [114]. Unfortunately, measuring the core temperature, e.g., by integrating sensors inside the cell, is still an unresolved challenge and thus a current research topic [115]. Another possibility is to use data or modeling based determination approaches to determine the core temperature of the individual cells. Furthermore, model-based approaches can be an important element of intelligent batteries in terms of their prediction functionality.

In the field of thermal modeling of conventional battery systems, various modeling approaches already exist, which use detailed 3D-electrochemical-thermal (e.g., [116]) or electro-thermal models (e.g., [117]). Detailed 3D-models based on electro-thermal coupling in any form are well-suited to analyze the behavior in detail. However, they are not applicable for the use in a vehicle BMS and therefore inappropriate in an IBS due to their high computational effort. In contrast, simplified modeling approaches are an attractive option, if the relevant relations are implemented and the prediction accuracy is sufficient. In general, real-time approaches can be organized and compared according to the modeling approach, the model's dimension, and the form of the modeled cell.

Simple mathematical models and Thermal Equivalent Circuit Models (TECMs) are widely used for the thermal modeling of small cylindrical cells [118-121]. In the field of TECMs, Forgez and Damay introduce models for a cylindrical cell [122] and a large prismatic cell [123] to resolve the transient differences between outside and inside temperature. Comparable TECMs with one to three thermal masses and cell individual temperatures are commonly used for system modeling with a large amount of cells [99]. Increasing the level of detail, both Zhao et al. [124], for pouch cells, and Li et al [125], for prismatic cells, publish models with an increased number of thermal masses per cell. Their models are therefore capable of resolving even the local temperature distribution. Certainly, primarily the level of detail defines the calculation time and needs to be selected with regard to the application. Observer structures can also be used to estimate the core temperature with thermal reduced order models using a surface measurement, as in [126,127], for example.

A different approach to physics-based thermal modeling is the data-driven approach where appropriate datasets already include the information of the hardware influence and interactions. Completely data-driven modeling approaches for conventional cells are implemented in the literature for example by learning systems. Kleiner et al. [128,129] and Panchal et al. $[130,131]$ show how data-driven ANN models can predict the thermal behavior of Li-ion cells in real-time. First, comparisons for the optimal architecture of the adaptive system are made in $[128,132]$ with the result that ANNs for time-series prediction are better suited than simple feedforward networks.

There is one aspect that must not be forgotten when implementing thermal models for IBSs which is the additional hardware components. If additional components are added to the cell or system to be modeled, the thermal interactions need to be represented. A first investigation with electronics representation is shown in [129]. Thereby, an intelligent cell is modeled either with a TECM or an ANN with the result that both approaches need to represent the electronics influence with different advantages in accuracy and implementation effort of the models. 
A totally different data-driven approach is based on current and voltage measurements and their evaluation via Electrochemical Impedance Spectroscopy (EIS). The EIS-based methods are supposed to represent the core temperature non-invasively, faster, and more accurately than externally placed temperature sensors $[133,134]$. Temperature indication methods based on EIS rely on the premise that the electrical behavior of battery cells is heavily temperature-dependent. By knowing the impedance spectrum, or parts of it, an average core temperature can be deduced based on characteristic reference measurements. The underlying temperature models correlate impedance with temperature and are very similar to those of the model-based temperature prediction. Therefore, several researchers combine both approaches [121,135].

Table 3 provides an overview of the published research in the last decade, starting with the pioneering work of Srinivasan et al. $[133,136]$ for single frequency EIS measurements. In this overview, the common case, where no cell failures occur, is studied and summarized. Nota bene, recent work of Srinivasan et al. [137] also evaluates the method in conjunction with thermal runaway. There exist several other recent reviews and comparison works on the topic of EIS-based temperature sensing $[134,138,139]$. In contrast, we strongly focus on recently proposed variants that were tested with onboard equipment or at least consider onboard conditions and issues in their investigations. The methods are categorized by the therein incorporated features. Since the impedance $\underline{Z}(j \omega)$ is a complex number, its representation is either in polar $(|\underline{Z}|, \arg (\underline{Z}))$ or Cartesian coordinates $(\Re(\underline{Z}), \Im(\underline{Z}))$. As shown in Table 3, the most common approach is to use $\arg (\underline{Z})$, which is assumed to enable measurements that depend only slightly on SOC, State of Health (SOH) $[140,141]$, and cell capacity [137]. Additionally, $|\underline{Z}|$ can be subject to higher production fluctuations [142], which reduces the reference models' generalization abilities. Beelen et al. $[138,139,143]$ combine both $\Re(\underline{Z})$ and $\Im(\underline{Z})$, whereby the scaling between both features is optimized by Monte Carlo simulations. An alternative approach is to perform a principal component analysis to transform multi frequency impedance data into a compressed vector space, which, to the authors' knowledge, has not yet been described in the literature. Raijmakers et al. and Ranieri et al. use the frequency that is needed to hit an imaginary value of $0 \Omega$ [144-146] or $650 \mu \Omega[145,147]$ as model input for temperature estimation. Thus, the excitation frequency has to be swept between at least two values to interpolate the desired crossover-frequency. The imaginary impedance value is chosen in a way that the load current distortions get negligible for automotive applications (see Section 3.3 for further information on onboard impedance estimation). In addition to measurement errors that are caused by non-synchronous current sensing with superimposed load current, model errors occur too [143]. Since the reference models were trained at equilibrium, missing relaxation times due to load currents before and during the measurement lead to estimation errors. While Socher et al. [148] conclude that the influence may be negligible under certain operating conditions, Zhu et al. propose a compensation technique, whereby the deviations are eliminated by incorporating an exponential correction function depending on the relaxation time [141]. Since the load current changes dynamically in Electric Vehicles (EVs), an appropriate relaxation time cannot simply be determined and the consequences on temperature estimation accuracy are not clear.

In summary, the influence of electronics and its representation in thermal models is an important aspect that has been poorly represented in the literature and is discussed in Section 5.4.1. Regarding EIS-based approaches, there is little published research $[145,147]$ that evaluates the method in a real-world test and compares it to conventional temperature measurement. 
Table 3. EIS-based approaches for the identification of the internal temperature in alphabetical order. Abbreviations: Lithium Titanate Oxide (LTO).

\begin{tabular}{|c|c|c|c|c|c|c|c|}
\hline & \multicolumn{5}{|c|}{ Features } & \multirow{2}{*}{ Frequencies } & \multirow{2}{*}{ Cell } \\
\hline & $|\underline{Z}|$ & $\arg (\underline{Z})$ & $\Re(\underline{Z})$ & $\Im(\underline{Z})$ & $f$ & & \\
\hline Beelen et al. [138,139] & & & $x$ & $x$ & & $50 \mathrm{~Hz}$ & $90 \mathrm{Ah}$ LFP \\
\hline Beelen et al. [143] & & & $\mathrm{x}$ & $\mathrm{x}$ & & $133 / 630 \mathrm{~Hz}$ & 23 Ah NMC \\
\hline Carkhuff et al. [149] & & $\mathrm{x}$ & & & & $\begin{array}{l}\text { Ano.: } 70 \mathrm{~Hz} \\
\text { Cath.: } 10 \mathrm{~Hz}\end{array}$ & $5.3 \mathrm{Ah} \mathrm{N} / \mathrm{A}$ \\
\hline Haussmann et al. [150] & $x$ & & & & & $500 \mathrm{~Hz}$ & $26 \mathrm{Ah}$ N/A \\
\hline Morello et al. [151] & & $\mathrm{x}$ & & & & $1 \mathrm{kHz}$ & 34 Ah NMC \\
\hline Raijmakers et al. $[144,145,147]$ & & & & & $x$ & $300-400 \mathrm{~Hz}$ & 2.3 Ah LFP, 7.5 Ah NCA, 90 Ah LFP \\
\hline Raijmakers et al. [145,147] & & & & & $x$ & $1.6-2 \mathrm{kHz}$ & 90 Ah LFP \\
\hline Ranieri et al. [146] & & & & & $\mathrm{x}$ & $80 \mathrm{~Hz}-2 \mathrm{kHz}$ & 12 Ah NCA \\
\hline Richardson et al. [120,135] & & & $\mathrm{x}$ & & & $215 \mathrm{~Hz}$ & 2.3 Ah LFP \\
\hline Richardson et al. [152] & & & & $x$ & & $215 \mathrm{~Hz}$ & 4.4 Ah LFP \\
\hline Schmidt et al. [153] & & & $\mathrm{x}$ & & & $10.3 \mathrm{kHz}$ & 2 Ah LCO \& NCA \\
\hline Schwarz et al. [154] & & $\mathrm{x}$ & & & & $1 \mathrm{kHz}$ & $20 \mathrm{Ah}$ LTO \\
\hline Socher et al. [148] & & & $\mathrm{N} / \mathrm{A}$ & & & $\mathrm{N} / \mathrm{A}$ & $21 \mathrm{Ah}$ NMC \\
\hline Spinner et al. [155] & & & & $\mathrm{x}$ & & $300 \mathrm{~Hz}$ & $2.6 \mathrm{Ah} \mathrm{LCO}$ \\
\hline Srinivasan et al. [133,136] & & $\mathrm{x}$ & & & & $40 \mathrm{~Hz}$ & 2.3 Ah LFP, 4.4 Ah N/A, 50 Ah LCO \\
\hline Srinivasan et al. [137] & & $\mathrm{x}$ & & & & $5 \mathrm{~Hz}$ & $50 \mathrm{Ah}$ LCO, 5.3 Ah N/A, 3 Ah N/A \\
\hline Wang L. et al. [156] & & $\mathrm{x}$ & & & & $12 / 44 / 79 \mathrm{~Hz}$ & $1.3 \mathrm{Ah}$ LFP \\
\hline Wang X. et al. [157] & & $x$ & & & & $79.4 \mathrm{~Hz}$ & 8 Ah LFP \\
\hline Wang X. et al. [158] & & $\mathrm{x}$ & & & & $10 \mathrm{~Hz}$ & 8 Ah LFP, 40 Ah LFP \\
\hline Zhu et al. [140] & $x$ & $x$ & & & & $\begin{array}{c}10-100 \mathrm{~Hz} \\
600 \mathrm{mHz}-17 \mathrm{~Hz}\end{array}$ & $\begin{array}{l}8 \mathrm{Ah} \mathrm{N} / \mathrm{A} \\
8 \mathrm{Ah} \mathrm{N} / \mathrm{A}\end{array}$ \\
\hline Zhu et al. [141] & & $x$ & & & & $10 \mathrm{~Hz}$ & $30 \mathrm{Ah}$ LFP \\
\hline
\end{tabular}

\subsection{Online Identification of State of Charge}

The determination of the State of Charge (SOC) is commonly regarded as one of the main tasks of the BMS to avoid overcharge, deep discharge, and reliable driving range prognosis. Generally, various possible approaches exist to determine the SOC that were surveyed in a number of reviews over the last years $[21,159,160]$. However, most of the contained contributions focus on a single Li-ion cell, whereas today's battery systems consist of multiple cells connected in parallel and serial strings. Only a few publications, which are discussed in the following, have addressed this topic so far.

Plett et al. [161] introduce an approach named bar-delta filtering where firstly an average SOC is estimated (bar), and secondly the difference of each cell from the average (delta) is considered. Roscher et al. propose a similar method in [162] using Lithium Iron Phosphate (LFP) chemistry, which is challenging due to the flat increase of the OCV with the SOC. The system's SOC is estimated with a Luenberger observer, and, for each cell, a factor is adjusted online, which represents the divergence to the average of all cells. It is further shown for Lithium Manganese Oxide (LMO) as well as for LFP cells [163,164] that multiple EKFs can be utilized for the average SOC determination and for cell individual SOC divergence. Following this, Sun et al. [165] focus on serial connected Lithium Nickel Manganese Cobalt Oxide (NMC) cells and emphasized the need for efficient algorithms for large battery systems. The approach of Yang et al. [166] is similar and focuses on serial strings only. 
A substantially different method is proposed by Zhong et al. [167], where the LFP battery pack's SOC is determined with respect to the strongest and the weakest cell. The authors state that the individual SOCs are less important for the battery's total SOC than the difference between the two cells with the highest and lowest SOC. To conclude, the SOC determination is difficult in battery packs if current and voltage are not measured individually for each cell. However, individual current information is difficult to obtain in ordinary battery systems in particular with cells connected in parallel. At this point, we emphasize the inherent advantage of IBSs where SOC estimation techniques are implementable with ease because each cell features individual sensors (in particular for current, voltage, and temperature). Therefore, a wide range of estimation approaches which were described in recent years for single cell application are adaptable for an IBS. A very simple approach is integrating the current measurement over time, which is known as coulomb counting. However, this approach is prone to current sensor bias errors that accumulate over time. Otherwise, SOC estimation based on OCV, as applied in $[168,169]$, requires periods of low current and is usually much slower.

Most commonly, state observers [170,171], sliding-mode observers [172,173], or KFs and their variations are applied for SOC estimation, which usually are based on an ECM of the Li-ion cell. EKFs are mostly used with simple ECMs (see Figure 3) [174-177]. Sometimes, the model is enhanced by electro-chemical equations based on the Single Particle Model (SPM) [178-180]. However, the model accuracy significantly influences the estimation performance $[101,103,181-183]$ as does the measurement accuracy [110,181]. Most recently, SOC estimation is combined with estimation of further states e.g., SOH [173,184-187] or State of Function (SOF) [188]. Otherwise, SOC determination is combined with the estimation of cell parameters like battery capacity $[100,189,190]$, internal resistance [191], or other model parameters [101,192,193].

Besides EKFs, Unscented Kalman Filters (UKFs) like the Sigma-Point KF are applied for SOC estimation [194,195]. Adaptive KFs are utilized too [196,197]. A distinction is made between joint KFs, where the state vector is augmented with the parameter vector, and DKFs, where two KFs run in parallel, one as the state observer and one as the parameter estimator. Furthermore, instead of combining only KFs, RLS algorithms [102,125,198] or PFs [199] are also applied for parameter estimation. PFs are rather unsuitable for IBSs though due to their high computational effort.

A more promising approach is SOC identification with ANNs as proposed by [200-203] or Support Vector Regression (SVR) [204,205]. Training data sets of sufficient size must be provided to exploit these methods. It has been shown by Ozean et al. [206] that Gaussian Process Regression (GPR) can be also used for state estimation.

In contrast to the previous presented approaches that operate in the time domain, in $[207,208]$, SOC estimation is based on EIS. Here, it should be noted that the required sampling rate corresponds to the desired frequency range of the impedance spectrum, which usually is considerably higher than for methods in the time domain.

While usually only electric quantities are considered, intelligent battery cells sometimes are equipped with additional sensors. The relation between cell pressure and SOC has come into focus in the last few years [209,210]. Ganguli et al. added fiber-optic sensors for pressure and temperature measurement in Li-ion cells and utilize the additional sensor data for improved SOC estimation [211]. Similar approaches are pursued by Ghannoim et al. [212,213] and Modrzynski et al. [214]. This topic will be further discussed in Section 3.5 from a hardware perspective.

\subsection{Online Identification of State of Health}

Compared to SOC estimation, online determination of State of Health (SOH) is generally more challenging and indistinct due to ambiguous definitions of $\mathrm{SOH}$. With regard to the capacity, $\mathrm{SOH}$ is most commonly defined as the ratio between currently available

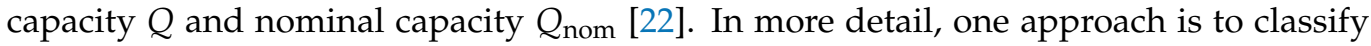
the available capacity loss into Loss of Lithium Inventory (LLI) and Loss of Active Mate- 
rial (LAM) [215], e.g., by Differential Voltage Analysis (DVA) [216]. Contrarily, concerning available power, which is of higher interest regarding hybrid electric vehicles, the increase of the ohmic resistance $R_{0}$ is regarded as $\mathrm{SOH}$ [217]. The growing Solid Electrolyte Interphase (SEI) causes the degradation effect in this case. Several reviews dealt with online $\mathrm{SOH}$ estimation in recent years [22,218-220], where [221] focuses on machine learning methods only. A broad discussion of the methods is omitted in this article and only the most promising approaches, for the application in IBSs in EVs, are discussed in more detail.

Table 4 gives an overview of relevant contributions on $\mathrm{SOH}$ determination. Most model-based approaches are based on ECMs, whereas physical models or SPM are scarcely utilized. Using observers, like the popular KF, for SOH estimation mostly aims to determine the capacity directly. In contrast, data-driven methods like ANN or SVR require prior testing to generate training data. The same is true for empirical methods where features are linked with data from prior aging tests. To which extent the results of such tests are applicable to other cell chemistries is questionable. Some researchers rely on features like diffusion time [222], Constant Current Charging Time (CCCT) [223,224] or Constant Voltage Charging Time (CVCT) [224,225], or focus on features embedded in the OCV by applying DVA/Incremental Capacity Analysis (ICA) [226-229]. Here, it should be stressed that these approaches require periodic charge processes to be applicable.

Of special interest is SOH identification based on EIS as it facilitates a quick determination procedure, especially when considering just one frequency that is sensitive to degradations [142,230,231]. These methods are promised to be applicable on-board in an IBS.

With regard to cell individual SOH estimation, Lajara et al. [232] focus on simple algorithms with low computational costs. They address the problem of wireless sensor networks, where multiple battery systems operate simultaneously with limited computing power. Ganeshan et al. [200] implement an algorithm that calculates SOC and SOH with coulomb counting as well as an ANN on a low-budget 8 bit-Microcontroller Unit (MCU), which emphasizes the need for algorithms that work with low computing power.

Finally, approaches based on additional sensors are discussed. Ganguli et al. [211] embed a fiber-optic sensor into a Li-ion cell to measure strain and temperature. By conducting aging tests, a correlation between $\mathrm{SOH}$ and a certain wavelength at the end of charge is found. Furthermore, Gong et al. [233] feature prismatic large format Li-ion cells (capacity of $20 \mathrm{Ah}$ ) with pressure sensors to detect cell internal gas production. As they report, capacity fade and internal pressure correlate with each other.

In conclusion, $\mathrm{SOH}$ estimation is a vibrant field of research. With more efficient model approaches and improved hardware, even sophisticated physics-based models might be introduced for EVs in the future as proposed by Li et al. [234]. While most methods are based on current and voltage sensor data, evaluation of further sensor domains like temperature or pressure is promising. The integration of these sensors is discussed in Section 3. However, even without additional measurement data, the use of cell voltage and current facilitates SOH determination in an IBS for each individual cell. This information is helpful for operating strategies (see Section 4). Degradation of single cells is usually invisible in conventional battery systems. 
Table 4. Online SOH identification approaches in chronological order. Abbreviations: Discrete Wavelet Transform (DWT), Level Shifted (LS).

\begin{tabular}{|c|c|c|c|c|c|c|c|c|c|c|c|c|}
\hline \multicolumn{4}{|c|}{ Modeling Approach } & \multicolumn{3}{|c|}{ Feature } & \multicolumn{6}{|c|}{ Methods } \\
\hline 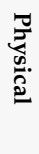 & 罙 & 悬 & 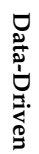 & 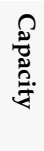 & $\sigma$ & 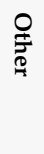 & 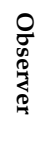 & 㐏 & $\underbrace{\infty}_{\infty}$ & 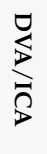 & $\frac{\pi}{\omega}$ & 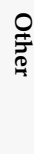 \\
\hline
\end{tabular}

\begin{tabular}{|c|c|c|c|c|c|c|c|c|c|c|c|c|c|}
\hline Troeltzsch et al. [230] & & & $x$ & & $x$ & $\mathrm{x}$ & & & & & & $x$ & \\
\hline Lee et al. [226] & & & & $\mathrm{x}$ & $\mathrm{x}$ & & & & & & $\mathrm{x}$ & & \\
\hline Haifeng et al. [217] & & & $x$ & & & $x$ & & DKF & & & & & \\
\hline Chiang et al. [235] & & & $\mathrm{x}$ & & & $\mathrm{x}$ & & Adaptive $\mathrm{O}$. & & & & & \\
\hline Kim et al. [19] & & & $x$ & & $\mathrm{x}$ & $\mathrm{x}$ & & $\mathrm{SMO}$ & & & & & \\
\hline Plett et al. [236] & & & $\mathrm{x}$ & & $\mathrm{x}$ & & & & & & & & WTLS \\
\hline Remmlinger et al. [237] & & & & $\mathrm{x}$ & & $\mathrm{x}$ & & & & & & & RLS \\
\hline Hu et al. [174] & & & $x$ & & $\mathrm{x}$ & & & DKF & & & & & \\
\hline Rahimian et al. [238] & & $\mathrm{x}$ & & & & & LAM & $\mathrm{EKF} / \mathrm{UKF}$ & & & & & \\
\hline Andre et al. [192] & & & & $x$ & $\mathrm{x}$ & & & & & $x$ & & & \\
\hline Feng et al. [227] & & & & $\mathrm{x}$ & $x$ & & & & & & $\mathrm{x}$ & & Point Counting \\
\hline Kim et al. [189] & & & & $x$ & $x$ & & & & $x$ & & & & \\
\hline Nuhic et al. [239] & & & & $\mathrm{x}$ & $x$ & & & & & $\mathrm{x}$ & & & \\
\hline Prasad et al. [222] & & $x$ & & & & $\mathrm{x}$ & Diffusion Time & & & & & & LS \\
\hline Remmlinger et al. [240] & & & $x$ & & & $\mathrm{x}$ & & KF & & & & & \\
\hline Schwunk et al. [241] & & & & $\mathrm{x}$ & $\mathrm{x}$ & & & $\mathrm{PF}$ & & & & & \\
\hline Weng et al. [242] & & & & $\mathrm{x}$ & $x$ & & & & & $\mathrm{x}$ & $\mathrm{x}$ & & \\
\hline Zheng et al. [243] & & & & $\mathrm{x}$ & $\mathrm{x}$ & & & & & & & & GA \\
\hline Eddahech et al. [225] & & & & $\mathrm{x}$ & & & CVCT & & & & & & Empirical \\
\hline Guo et al. [223] & & & $x$ & & & & СССТ & & & & & & NLS \\
\hline Han et al. [244] & & & & $x$ & $x$ & & & Calibrated O. & & & & & \\
\hline Hu et al. [245] & & & & $\mathrm{x}$ & & & Sample Entropy & & & & & & Empirical \\
\hline Kim et al. [80] & & & & $\mathrm{x}$ & & & DWT & & & & & & Empirical \\
\hline Zou et al. [185] & & & $x$ & & $x$ & & & DKF & & & & & \\
\hline Berecibar et al. [246] & & & & $\mathrm{x}$ & $x$ & & & & & & $\mathrm{x}$ & & \\
\hline Wu et al. [247] & & & & $x$ & $x$ & & & & $\mathrm{x}$ & & & & \\
\hline Zou et al. [186] & $x$ & & & & $\mathrm{x}$ & & & EKF & & & & & \\
\hline Dubarry et al. [248] & & & & $x$ & & & LAM, LLI & & & & & & Empirical \\
\hline Gong et al. [233] & & & & $\mathrm{x}$ & & & Gas Production & & & & & & Empirical \\
\hline Huhman et al. [231] & $\mathrm{x}$ & & & & $\mathrm{x}$ & & & & & & & $x$ & \\
\hline Sanchez et al. [249] & & Vesse & odel & & $x$ & & & & & & & & Fuzzy \\
\hline Cai et al. [250] & & & & $x$ & & & DWT & & & & & & Empirical \\
\hline Chen et al. [251] & & & & $\mathrm{x}$ & $x$ & & & & & & 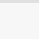 & & RF \\
\hline Lajara et al. [232] & & & & $x$ & $x$ & & & & $x$ & & & & LS \\
\hline Li et al. [228] & & & & $x$ & $x$ & & & & & & $\mathrm{x}$ & & \\
\hline Li et al. [252] & & & $x$ & & $\mathrm{x}$ & & & EKF, PF & & & & & \\
\hline Santos et al. [253] & & & & $\mathrm{x}$ & $\mathrm{x}$ & & & & $\mathrm{x}$ & & & & \\
\hline Shen et al. [198] & & & $x$ & & $\mathrm{x}$ & & & & & & & & RLS \\
\hline Smiley et al. [254] & $x$ & & & & $x$ & & & IMM KF & & & & & \\
\hline Tang et al. [255] & & & & $x$ & $x$ & & & & & & $x$ & & \\
\hline Wassiliadis et al. [256] & & & $\mathrm{x}$ & & $\mathrm{x}$ & & & DEKF & & & & & \\
\hline Ganeshan et al. [200] & & & & $x$ & $\mathrm{x}$ & & & & $\mathrm{x}$ & & & & \\
\hline Yu et al. [257] & & & $\mathrm{x}$ & & $\mathrm{x}$ & & & $\mathrm{H}_{\infty}$ & & & & & \\
\hline Zheng et al. [258] & & & $x$ & & $x$ & & & DKF & & & $x$ & & Arrhenius \\
\hline Bi et al. [259] & & $x$ & & & $\mathrm{x}$ & $\mathrm{x}$ & LAM, LLI & $\mathrm{PF}$ & & & & & \\
\hline Jiang et al. [260] & & & & $x$ & $\mathrm{x}$ & & & & & & $\mathrm{x}$ & & \\
\hline Liebhart et al. [142] & & & & $\mathrm{x}$ & $x$ & & & & & & $x$ & & \\
\hline Liu et al. [224] & & & & $x$ & $x$ & & CVCT, CCCT & & & & & & Empirical \\
\hline Maletic et al. [190] & & & $\mathrm{x}$ & & $x$ & & & DKF & & & & & \\
\hline Meng et al. [104] & & & $x$ & & $\mathrm{x}$ & & & UKF & & & & & \\
\hline Shu et al. [261] & & & & $\mathrm{x}$ & $\mathrm{x}$ & & & & $\mathrm{x}$ & $\mathrm{x}$ & & & \\
\hline Xu et al. [262] & & & & $x$ & & & DWT & & & & & & Empirical \\
\hline Yang et al. [263] & & & & $\mathrm{x}$ & $x$ & & & & & & & & Empirical \\
\hline
\end{tabular}




\subsection{Online Identification of State of Function}

To evaluate the battery system's ability to fulfill its requirements regarding power, SOC and $\mathrm{SOH}$ are less valuable metrics. According to Meissner et al., both states compensate each other to a certain degree with respect to power output requirements [264]. Consider an aged battery which will, on average over SOC, have a higher internal resistance than a new one. This battery will need a higher OCV to achieve the same power output because of a larger voltage drop across the internal resistance, which is possible by adjusting the lower SOC-limit upward. Therefore, commonly the State of Function (SOF), also known as State of Performance (SOP), is used to assess the power that is deliverable by the battery instantaneously.

As state-of-the-art approach, the HPPC method is conducted for determination of power capability, which was published by the Idaho National Engineering and Environmental Laboratory of the U.S. Department of Energy [265,266], but the test procedure focuses only on not violating the upper and lower voltage limits. However, current, power, SOC, and temperature limits are essential as well.

Recent approaches to online SOF determination are summarized in Table 5. As can be seen, most methods refer to one or more limits. Most commonly, SOF evaluation is based on an ECM and the maximum current is predicted for certain time windows (e.g., $5 \mathrm{~s}$, $10 \mathrm{~s})$ [267]. However, the current is assumed to be constant over the length of the considered time window which may result in conservative SOF estimates during dynamic operation.

Table 5. Online SOF identification approaches. Abbreviations: Voltage (U), Current (I), Power (P), and Temperature (T), Model Predictive Control (MPC).

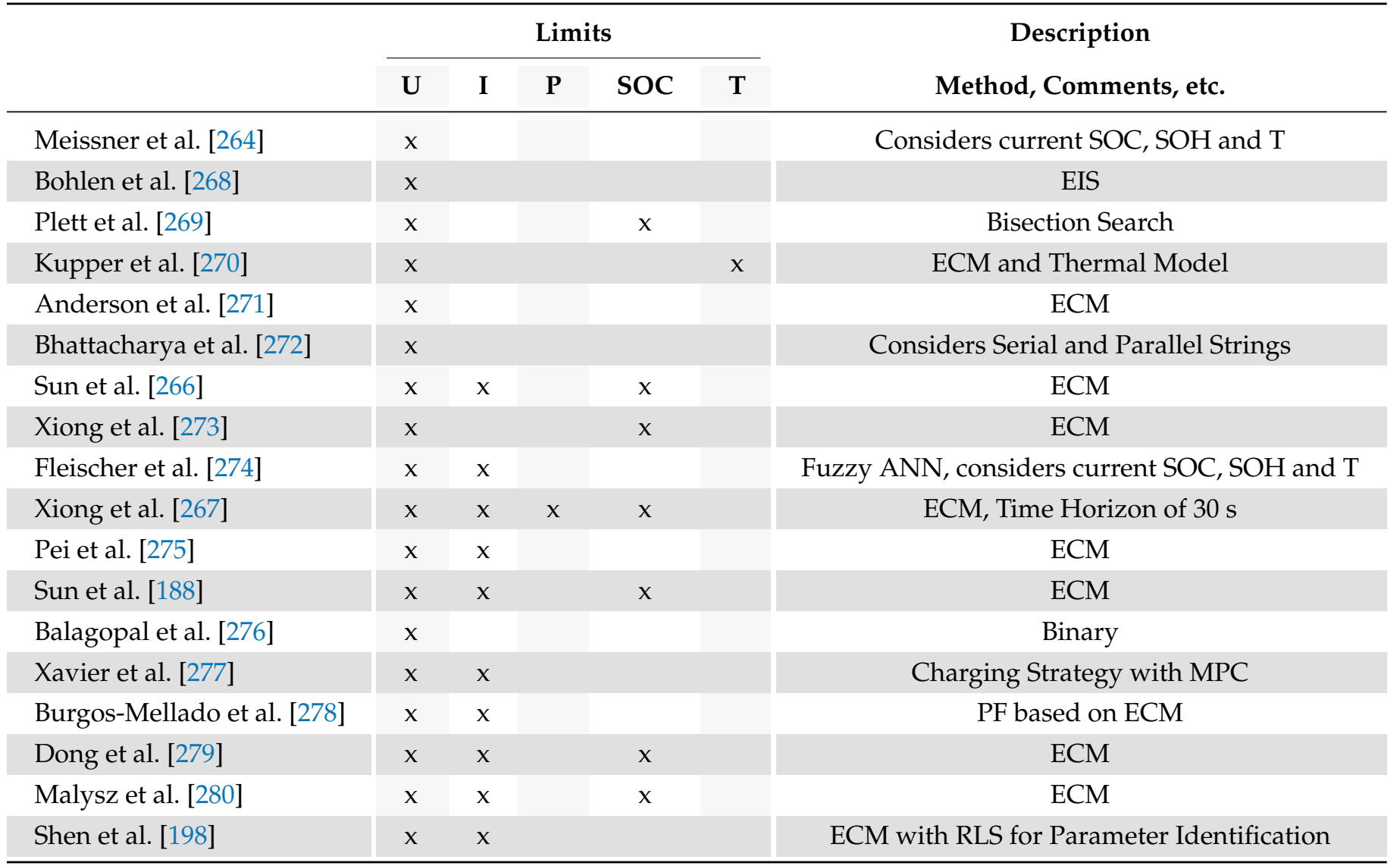

As the battery systems power capability is based on the capability of each single cell, their specific SOF must be taken into account. Instead, most commonly, the cells' states are assumed to be equal [272], which is an oversimplification over battery lifetime. 
An IBS promises the benefit of more accurate determination of the battery system's SOF when each individual power capability is considered. Furthermore, as we will point out in Section 4, operating strategies may take individual SOFs into account. Hence, SOF determination on cell level is a necessary basis of advanced operation strategies in an IBS.

\section{Implementation of Sensing in Intelligent Battery Systems}

The previous section has shown the extensive opportunities of advanced monitoring approaches under the premise of sufficiently available measurement data. Thus, the data acquisition with sensing on single cell level is considered to be a vital building block for the realization of an IBS, but at the same time its implementation represents a significant challenge. Hence, the implementation of sensor technology has to be considered carefully. In addition to most common sensor domains like current, voltage, and temperature, more sophisticated sensor technologies are promising to gain deeper insight into the cell's state. However, this advantage comes at the price of higher costs and complexity. The approaches range from the integration of sensors into the cell to the application of non-intrusive diagnostic techniques like EIS and acoustic methods.

In the following, we give an overview of sensors and sensor domains, which are considered as promising for the use in IBSs and point out practical implementation, obstacles, and open scientific questions. Furthermore, requirements with regard to the application of BEVs are discussed.

\subsection{Current}

Electric current is one of the most important quantities in battery applications. While conventional battery systems typically use one central current sensor, IBSs might use multiple smaller current sensors that are distributed throughout the system [281,282]. This provides additional data that can be used for advanced functions like error detection, sensor data fusion [283], or to improve parameter estimation [284]. Due to the increased number of sensors, the selection of current sensors in an IBS requires special consideration. This section will give a summary of important requirements for potential sensor candidates. A brief overview of suitable sensor principles is provided and promising methods are described.

In advance of selecting or developing a current sensor for an IBS, functional and nonfunctional requirements have to be defined: The sensor has to support the full range of the cell's charge and discharge current. Given the trend to cells with higher and higher capacity, this can range from tens to hundreds of amperes. Sensors in IBSs have to be able to measure DC but should also provide sufficient bandwidth for the frequency ranges that are required by certain monitoring functions. Features like EIS or Pulse Width Modulation (PWM) significantly increase the required bandwidth in comparison to conventional systems.

Additionally, there are various requirements concerning the quality of the obtained measurements. Measurement errors can affect functions like EIS as well as SOC and parameter estimation. This in turn leads to increased safety margins that limit overall performance. Static offset and gain errors are easily corrected in software, time, or temperature dependent error drift is a more complex problem. Furthermore, nonlinearity can impair the spectra acquired with EIS. These errors are difficult to compensate without time-consuming calibration. Sensor noise compromises all monitoring functions. Signal processing methods, like filtering and sensor fusion [283], help to reduce the effective noise power.

Research has been done on various current sensing techniques for a wide range of applications [23,285-287]. Many of these techniques can be used for IBSs in EVs. The suitability, however, greatly depends on the actual implementation of the system. In general, current sensing techniques are categorized into resistive or electromagnetic methods. While the former use a resistive element in the current path, the latter measure current by measuring the electromagnetic field around a conductor.

A well-known resistive technique is to use a Current Sense Resistor (CSR), also known as shunt resistor. Current sensing should not increase the parasitic resistance in the current path; therefore, low resistance CSRs is used, and the resulting low voltage 
signal must be amplified. While the resistor itself is accurate and linear, the amplification can impact measurement performance by introducing offset and gain errors as well as nonlinearity [286]. Either the resistor's parasitic inductance or the following amplifier may limit the bandwidth of this technique. High precision CSRs are made of special alloys with low temperature coefficients [286]. Additionally, temperature compensation may be used to achieve good accuracy over a wide range.

Alternatively, one can use existing resistive elements for current sensing such as wires or busbars. This reduces parasitic resistances but copper and aluminum, the typical materials, have much higher temperature coefficients in comparison to CSRs. For short conductor stretches, the low resistance makes the measurement of the resulting voltage drop challenging. The high temperature coefficient makes temperature compensation critical [286].

In IBSs with semiconductor actuators (see Section 4), switches can be used as a resistive element. For Metal-Oxide-Semiconductor Field-Effect Transistors (MOSFETs), this

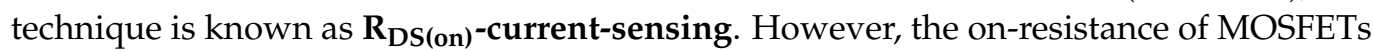
is highly temperature dependent [287] and subject to production tolerances of die, package, and connection to the circuit board. Temperature gradients inside the MOSFET increase the complexity. The relatively high on-resistance, which is unavoidable regardless of current measurement, makes this method a promising approach.

Another technique that relies on the on-resistance of MOSFETs, are Sense-FETs. This special type of MOSFET provides a measurement current, via an internal current mirror that is proportional to the primary current [287]. The current mirror ratio can be matched to the application. The instantaneous ratio depends on the temperature distribution on the die and the primary current. This makes precise measurement challenging [288].

While the aforementioned techniques require a galvanic connection to the primary circuit, electromagnetic based techniques are inherently isolated [287]. This is beneficial for high-voltage applications. A common technique is to combine a Hall-element with a magnetic core in order to measure the magnetic field around the conductor [287]. The core concentrates the magnetic flux at the sensing element and suppresses the influence of external fields.

Electromagnetic sensors tend to behave nonlinear and show a temperature dependent offset. To improve linearity, closed-loop sensors are applied. This sensor class uses a compensation winding to operate the sensing element at a constant flux [286]. This in turn increases power consumption.

The problem of offset drift is addressed by using better sensing elements. Fluxgate sensors are used in high-precision current sensors due to their extremely low offset [286]. Over the past few years, various types of magnetoresistive current sensors have also come onto the market but are still not widely adopted yet.

The demand for small size and high integration in an IBS makes coreless electromagnetic sensors an appealing solution. Differential sensor arrangements are used to suppress external fields [285]. Due to the confined space and resulting packing density in automotive battery packs, crosstalk between neighboring cells is to be expected nevertheless.

While a lot of research has been done regarding current sensors in general, the authors believe that additional research, targeting current sensing in IBSs, could lead the way to more cost-effective solutions. For example, approaches to use knowledge of IBS system behavior to compensate for errors, caused by low-cost sensors, with appropriate algorithms have not yet been explored. Currently, we believe that CSRs offers the best performance in a wide range of applications. In a system design that pursues maximum efficiency and minimum cost, this solution is still far from optimal. There is still a lot of work to be done in order to achieve an economic, energy efficient, and performant solution tailored to the demands and challenges of IBSs. 


\subsection{Voltage}

Cell individual voltage measurement is already implemented in conventional Li-ion battery systems. This is crucial for monitoring the upper and lower voltage limits of each cell to prevent failure and damage. Voltage measurements are also required by all advanced monitoring algorithms and are therefore an essential for IBSs.

In today's battery systems, the cell voltage is most commonly captured by integrated circuits, where the electrical signals are converted by ADCs and further processed by the BMS controller. Precise data acquisition is an acquainted challenge. Usually, the accuracy is limited to $1 \mathrm{mV}$, or more, for cost reasons. Higher measurement accuracy requires models and look-up tables of corresponding quality, which is usually not the case, to be beneficial. The trend to BMSs on cell level results in short wires. This is generally advantageous with regard to signal integrity and Electromagnetic Interference (EMI) [15].

Further requirements may arise depending on the applied functionalities in an IBS. The optimal sample rate depends on the algorithm that the measurement data are used for. For monitoring the safety voltage limits, a sampling time of $1 \mathrm{~Hz}$ or even less is sufficient. State and parameter estimation functions operate with sampling rates of $10 \mathrm{~Hz}-1 \mathrm{kHz}$. With regard to high frequency switching (for example in inverter concepts, Section 4.3), the switching frequency is in the range of many $\mathrm{kHz}$, which severely affects the measurement process.

For impedance measurement, current and voltage have to be acquired synchronously. Faced with a large amount of battery cells in the system, this task is challenging. Distributed measurement systems are favorable, where the measurement hardware and its processing is located close to each cell. In doing so, communication effort is kept low. Further thoughts on impedance measurement are presented in the following section.

\subsection{Impedance}

The literature review of advanced monitoring in Section 2 shows that functions such as $\mathrm{SOH}$ or SOF identification use impedance measurements in addition to cell voltage, current, and temperature. They require measurements either at single frequencies or over a range of frequencies also referred to as impedance spectrum. The metrological process of measuring the impedance spectrum of an electrochemical cell is commonly called Electrochemical Impedance Spectroscopy (EIS). However, in this section, the term is defined to include all methods used with the intent of measuring impedance at arbitrary frequencies.

Literature on EIS is a broad topic, which can roughly be divided into the subdomains of measurement, validation, and interpretation. A current review of the application of EIS [289] covers all these subdomains and may serve as a starting point. In this contribution, we will only review publications with a focus on the online implementation of EIS that include experimental results of the proposed method. These limitations are made with the intention of providing an overview of methods that are nearly applicable in IBSs.

The implementation of EIS requires to excite the cell, measure the response signal, and evaluate the acquired data. These tasks are particularly challenging for large battery systems with small impedance. Measuring small impedances requires either large excitation currents or sensitive, low-noise front-ends and data converters to be able to measure the system response with sufficient accuracy for further processing. To enable online measurements in commercial systems, the additional hardware costs should be kept to a minimum. This is especially critical when impedance measurement is aimed to be deployed on a module or cell level. The literature discussed in the following is further summarized in Tables S1 and S2.

Methods that estimate ECM parameters from time domain data also fit the broadened concept of EIS as the impedance of arbitrary frequencies can be extracted from the ECM. However, related publications were already discussed in Section 2.2 and will not be repeated here.

As the excitation source, linear $[149,157,290,291]$ and switched mode actuators [147,151,292-295] can be found in the literature. For cell level measurement, linear excitation is a simple and common technique, whereas switched mode actuators 
tend to be employed for large cells or packs to create arbitrarily shaped excitation signals. It is desirable to use either low-cost or already deployed hardware for this purpose. This includes, for example, the use of active [296] and passive [143,147,297,298] cell balancers, as well as more advanced methods such as using charging circuits [299,300] or the EV's traction inverter [207]. Switched mode actuators can be further differentiated based on whether the energy used to excite the system is dissipated as heat [301-303] or redirected to reduce power dissipation $[292,296,304]$. The latter is especially relevant for large battery systems, where high currents are necessary to obtain a sufficient voltage response. Omitting the actuators all together and measuring passively is also a viable option for many systems [112,268,293,304-310]. This, however, requires the load current to exhibit dynamic properties in order to be suitable as an excitation signal. Thus, in many cases, performing passive measurements limits the usable frequency range [112,293]. Switching events in an Reconfigurable Battery System (RBS) support the process of passive broadband impedance measurement in a comparatively efficient manner [293,294]. Sophisticated methods for excitation, using slightly modified existing hardware, are presented in $[290,296,304]$. The work of Gong et al. demonstrates a unique hybrid architecture, combining the benefits of linear and switched mode excitation [290].

Another challenging aspect of impedance measurement is the design of the Analog Front End (AFE) for data acquisition. Single-ended and differential AFEs comprising fixed or variable gain [292] amplifiers, Lowpass Filters (LPFs) for anti-aliasing, High Pass Filters (HPFs) for removing low frequency and DC components $[149,207,291,301]$, and more advanced circuits are commonly found in the literature. A notable implementation was described by Din et al. [296] using a digitally controlled DC servo amplifier for removing DC offsets from the voltage signal to utilize a larger part of the ADC's full-scale range. The utilized servo amplifier also features a fourth order active LPF for anti-aliasing. A CSR is most commonly used for current sensing [207,268,296,303,311], but Hall-effect current transducers are utilized as well [112,293,294,301,302]. Low-cost implementations use MCU internal SAR ADCs $[157,292,300,301,304]$ in most cases. For more performant systems, with an accuracy of $>12$ bit, standalone SAR or delta-sigma ADCs are employed typically [296,299,311-313].

Before transformation of the acquired data to the frequency domain, preprocessing is performed. Preprocessing steps may include, for example, digital filtering [268,290,314], gain and offset compensation [290,292], segmentation [112,315], windowing [112,306,307,316], zeropadding [308], and down sampling [293,296,312]. Potential uses of digital filtering include HPFs to remove low frequency components [290] and compensate for drift [314], Bandpass Filters (BPFs) as preparation for correlation based postprocessing [268] as well as decimation filters for down sampling $[293,296]$.

For online impedance estimation, especially the usage of Discrete Fourier Transformation (DFT) based methods is widely accepted and applied in many publications. There are also publications on time domain $[268,291,317]$ and Laplace based $[318,319]$ methods. Laplace based methods, however, tend to suffer from high computational complexity and are therefore challenging to implement on the resource constraint hardware of an online measurement system [319]. The impedance is calculated based on Power Spectral Density (PSD) and Cross Power Spectral Density (CPSD) [112,207,292-294,320] or by directly using the DFT of current and voltage [299,302,321]. PSD based approaches tend to yield better results as they are less susceptible to noise and distortion. Such frequency domain methods have not changed significantly since their introduction in 1982, when Osaka et al. proposed the usage of the DFT for online voltammetry in analytical chemistry [322]. Depending on implementation and hardware platform, these calculations may impose a significant computational burden.

To deal with this limitation, alternative approaches have been developed. Particularly unconventional methods are proposed by Carkhuff et al. who use a root mean square converter for magnitude measurement and an MCU timer peripheral as a phase detector [149]. Wang et al. calculate the impedance magnitude from the peak-to-peak ratio of 
the recorded current and voltage signals and the impedance argument from their relative temporal offset [157]. These approaches, however, are best suited for sinusoidal excitation and the absence of load current or other disturbances. More robust alternative approaches are based on correlation of current and voltage in a selected frequency range [268], the Goertzel algorithm [151,308,314], or single frequency DFT methods [296,300,311].

After impedance estimation, averaging may be utilized to reduce result variation and allow for more accurate tracking of impedance changes over time [315]. Window averaging [301], exponential averaging [297,306,315], and more advanced methods [305] are proposed in the literature.

With cell level measurement in a multi cell system, the choice of a distributed architecture with central excitation seems natural [151,290,310,311]. If the current measurement is also performed centrally, a reduction in electronics cost is achieved. This, however, requires a method to synchronize current and voltage measurement. Synchronization methods, based on communication or radio signals, are proposed in [290,310,311]. In addition to potentially reducing costs, the use of a distributed system allows for decentralized impedance estimation, which can offload calculations from the main BMS and also reduce data traffic in the communication system.

We draw a couple of conclusions from the present literature review. Regarding methods for impedance estimation, no clear recommendations can be made since the usability of the respective methods depends heavily on factors like excitation signal and method, system architecture, or degree of disturbance during the measurement.

Distributed systems have shown good results for impedance measurement. They require a mechanism for synchronizing the acquisition of voltage and current. The synchronicity has a large impact on the precision of the impedance argument at higher frequencies [311]. Further research in synchronization methods for BMS might therefore be worthwhile as solutions for similar problems exist in other fields. Passive EIS was shown to be feasible, but limitations of the instantaneous load current bandwidth have to be addressed by using hybrid approaches that combine passive and active excitation $[293,294]$. If the computational complexity and disturbance susceptibility of Laplace-based processing were overcome, implementations using impulse excitation could provide a promising alternative to Fourier-based approaches. This would enable the accurate transformation of transient processes, without the need for windowing and the consequential distortion of the results [318,319]. Averaging is shown to positively influence measurement quality and is therefore recommended, especially for measurements with low SNR [315]. It can be performed on frequency domain data as well as impedance data. To further reduce cost and enable compact solutions, chip-level integration of dedicated battery EIS hardware, combining multiple components of the signal path on a single chip, needs to be promoted [290,310].

The complexity of obtaining accurate impedance data is largely influenced by the cell under test. Measurements on small, high energy density cells with relatively high impedance are significantly easier to perform, since the system's response to a given excitation current will be higher compared to large, high power density cells. By varying environmental factors, like ambient temperature, the cell's impedance can be further influenced to suit the measurement system and enhance the experimental results. The reader is therefore advised to consider the magnitude of the impedance as an indicator for the intricacy of performing the measurement rather than the cell type or measurement conditions.

Given the multitude of factors that influence the measurement quality of EIS, it is clear that a holistic approach that considers all steps of the measurement process must be taken to bring online EIS to the module or even cell level.

In summary, the present literature review leaves the impression that the online estimation of battery impedance is feasible for applications, even though there is not a proven, almost standard set of approaches, yet, and it still requires significant effort. If the application of online impedance information results in clear benefits, the effort is justified, and there is enough research to point early adopters in a promising direction. 


\subsection{Temperature}

It is well known that the temperature is one of the most crucial parameters for safe and reliable operation of Li-ion cells. In state-of-the-art BEV, a limited number of temperature sensors are distributed within the battery, providing information to the BMS.

Due to their low cost and robustness, thermo-resistive devices such as thermistors with Negative Thermal Coefficient (NTC) are mostly used for temperature measurements in Li-ion EV batteries and portable devices [323,324]. Thermo-junctive devices (thermocouples), based on the Seebeck effect, are also broadly used in multiple studies [325,326] exhibiting good reliability and decent accuracy. Other methods such as thermal imaging [327,328], Johnson noise [329,330], and liquid crystals, despite their benefits, are too pricy and/or too bulky to be used in mass applications.

However, to improve the safety and the overall efficiency of the battery systems, the rising number of fails and fires reveals the necessity of cell individual temperature monitoring [134]. Therefore, in recent years, a significant amount of scientific work was conducted to develop new approaches and sensors for temperature monitoring in $\mathrm{Li}$-ion battery systems.

Various studies show that, due to the large thermal mass and the anisotropic thermal conductivity of the jelly roll the difference of internal and external temperatures can be significant, especially at high currents $[325,328,331]$. As a consequence, the internal cell temperature is often underestimated by external temperature measurements [332].

A rising number of scientific works focus on integration of temperature sensor in the Li-ion cells. Although cell internal sensors provide technically significant benefits for the safe and reliable operation of Li-ion cells, their implementation proves to be challenging and costly. Several requirements have to be fulfilled for sensors to be successfully integrated within commercial Li-ion cells for BEVs:

- low costs;

- $\quad$ small size, so that the volumetric energy density of the cell is not significantly reduced

- $\quad$ long term chemical stability towards the electrolyte;

- $\quad$ negligible impact on the cell performance;

- compatible with the cell assembly process;

- reliable operation at least for eight to ten years depending on the local geopolitical regulations.

Thin film temperature sensors based on thermistor and thermocouple technology are low cost and small-sized, however, need to be isolated from the aggressive liquid electrolyte of the Li-ion cells. Thereby, the used materials as well as the processing methods need to be considered for reliable and reproducible long term operation. Several studies use polyimide materials as support material or protective coating due to its very good thermal conductivity and chemical stability in polar aprotic solvents [333-335].

Mutyala et al. [334] reported a technique to fabricate a flexible thin film K-type thermocouple device embedded in polyimide and transferred to a thin copper foil. The sensor, which was attached to the outer face of the jelly roll of a $3 \mathrm{Ah} \mathrm{NMC/graphite} \mathrm{pouch} \mathrm{cell,}$ showed reliable behavior during the charging and discharging of the cell at different C-rates (see Figure 5a). 

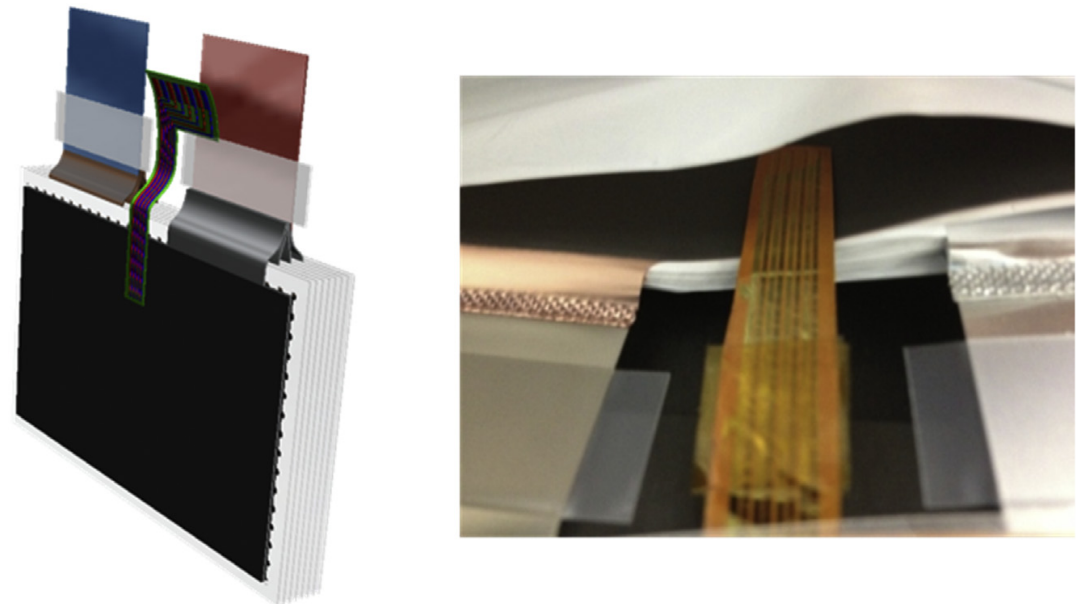

(a) Thermocouple @ Elsevier 2014

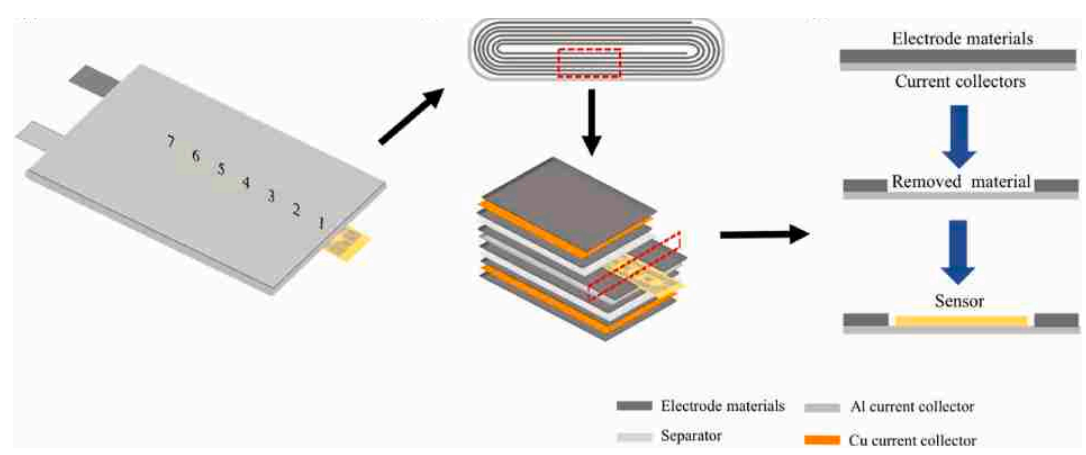

(c) Resistance Temperature Detector (RTD) @ Elsevier 2020

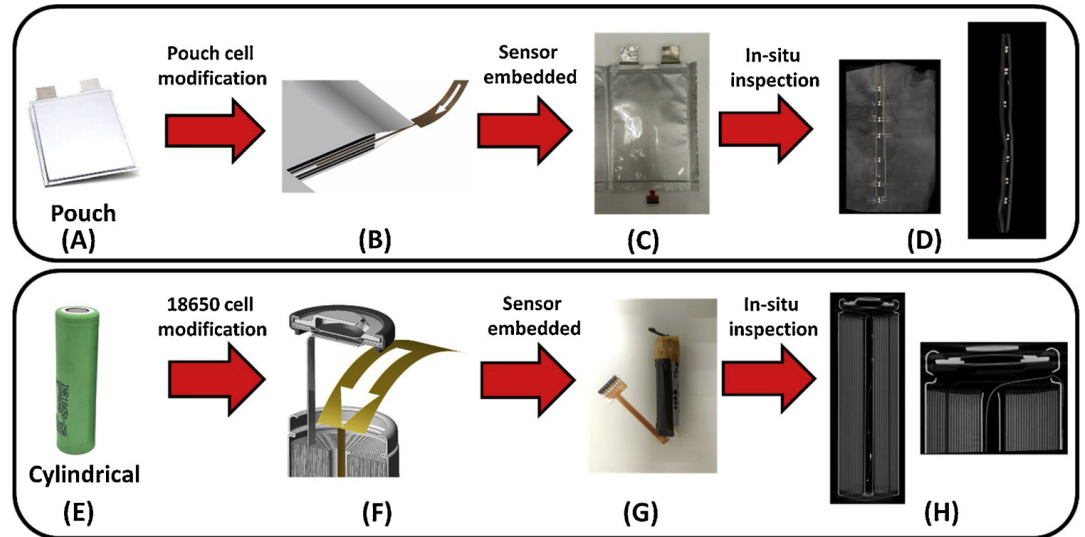

(b) NTC Thermistors $\odot$ Elsevier 2019
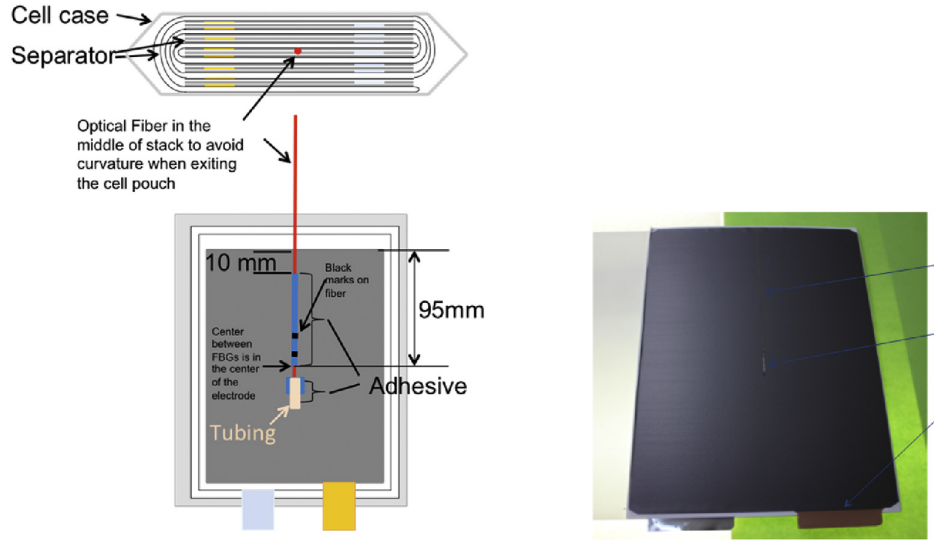

Polyamide
coated fiber

Tubing

Anode

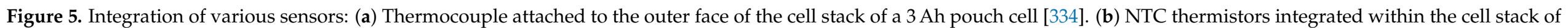

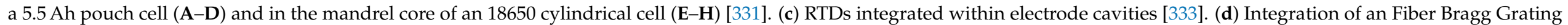
Sensor (FBG) within the cell stack of a 15 Ah pouch cell [336]. All reprinted with the permission of Elsevier, 2021. 
However, the authors do not report behavior and stability of the sensor over continuous cycling. Martiny et al. use a polyimide substrate to fabricate thin K-type thermocouple sensor assembly $(54 \mu \mathrm{m})$ by sputtering $\mathrm{Ni}$ and $\mathrm{Cu}$. Subsequently, the sensor, covered by a polyimide tape, is inserted in a 2 Ah Lithium Cobalt Oxide (LCO)/graphite cell [335]. The authors report that, while no influence from the sensor on the cell response is observed, the behavior of the sensor after few cycles is unreliable and irreproducible, most probably due to electrolyte leakage. The same group reports similar behavior for $27 \mu \mathrm{m}$ sensors using as a protective Parylene $\mathrm{C}$ coating, deposited via vapor deposition polymerization [337]. After the cell disassembly, damaged coating as well as massive corrosion of the $\mathrm{Cu}$ is observed. Although Parylene $\mathrm{C}$ exhibits high chemical stability and forms thin pinhole free coatings, its poor adhesion in liquid environments is generally an issue [338]. Fleming et al. [331] report a stable behavior of NTC thermistors with Parylene C protective coating, integrated directly into the mandrel core of $3 \mathrm{Ah}$ Lithium Nickel Cobalt Aluminum Oxide (NCA)/graphite 18650 cell as shown in Figure 5b. After the attachment of the thermistor to a polyimide substrate, the authors apply $1 \mu \mathrm{m}$ Parylene $\mathrm{C}$ coating via vacuum deposition. According to the authors, the integrated sensors are durable and have no adverse effect on the cell performances for the studied period of three months. The authors report similar behavior when the sensor is integrated between the middle layers of the jelly roll of a $5.5 \mathrm{Ah} \mathrm{LCO/graphite} \mathrm{pouch} \mathrm{cell} \mathrm{(see} \mathrm{Figure} \mathrm{5b).} \mathrm{Although} \mathrm{the} \mathrm{authors}$ do not observe an impact of the sensor integrated in the middle of the pouch cell, it is to be expected that the sensor would impede the ionic flow at this position between the electrodes, especially when the cells are compressed within a module.

Zhu et al. [333] suggest the integration of several thin polyimide embedded platinum RTDs in specially created cavities within the electrodes, so that the ionic flow is not disturbed (Figure 5c). The cells show a stable behavior over 100 charge/discharge cycles; however, a slight reduction of the cell capacity is apparent due to removal of active material.

\subsection{Mechanical and Volumetric Change Detection}

Another important parameter that is changing during the operation of Li-ion cells, is the mechanical stress induced in the cells by the main reversible intercalation and deintercalation of Li-ions and the side reactions. Due to lithium exchange between the electrodes, the active material contracts and expands. This effect is called breathing and is directly related to the lithiation of the electrodes. Irreversible aging effects, such as SEI growth and electrolyte decomposition, cause permanent expansion of the cell, called swelling. Additional information about the mechanical changes within the cell has the potential to significantly improve the in operando determination of SOC and SOH of the individual cells in the battery system. It can also support the early detection of safety critical events [339-342]. The general methods that are used for monitoring mechanical effects in individual Li-ion cells are summarized in two recent reviews [343,344].

In real applications, the volume changes are impeded by mechanical constraints, such as cell and module housing, inducing strain within the cells [343]. Few studies suggest the combination of thermal and strain sensors to simultaneously monitor the temperature and the mechanical changes within the cell during operation for improved and efficient assessment.

Knobloch et al. [345,346] report the design of a thin sensor platform containing platinum RTDs for temperature measurements and a flat spiral eddy current displacement sensor. The sensor platform is attached to the housing of prismatic $5 \mathrm{Ah}$ NMC/graphite cells and the cells are assembled into modules, so that the sensor platforms are positioned in between the cells [345]. Moreover, the authors instrument a 2014 Hybrid Electric Ford Focus battery pack, containing 76 compressed cells, with 19 sensor platforms [346]. Although the authors observe the expansion and contraction of the cells during charging and discharging, only the deflection of the cell in the lower SOC range is measured, due to the resolution of the eddy current sensor and the low displacement of the hard cased cells under compression. Nevertheless, the authors report that the cell individual RTDs detect the temperature changes better than the few original thermistors in the battery pack. 
Hu et al. [26] prepare a health monitoring sensor consisting of piezoelectric/pyroelectric polymer array (vinylidene fluoride-trifluoroethylene) and a thin-film transistor array by an in situ poling process. Attached to a Li-ion cell, the polymer array provides a real-time response to externally applied mechanical and thermal changes while the thin-film transistor array responds to quasi-static mechanical damage with a high resolution of $50 \mu \mathrm{m}$. According to the authors, the suggested monitoring sensor can be used as an early warning of mechanical and thermal damage.

Another popular approach for measuring the mechanical changes and the temperature in Li-ion cells is the integration of Fiber Bragg Grating Sensors (FBGs) [347]. FBGs are light-weight, small-sized, tolerant to EMI, and electrically insulating which makes them very attractive for application as internal sensors in Li-ion cells. FBGs are sensitive to temperature changes that influence the refraction index and cause thermal expansion of the optical fiber. Moreover, the Bragg wavelength is affected by strain due to physical elongation of the FBG and photo-elastic effects. Thus, many studies utilize the sensitivity of the FBG to simultaneously monitor the temperature and the surface or internal strain of Li-ion cells.

Several works claim that the integration of FBGs into the mandrel core of cylindrical cells is a promising method for in situ monitoring of the internal temperature [348-350]. However, it needs to be verified that the integrated sensor of the cell is not blocking the mandrel. Clogging the mandrel will increase the risk of cell bursting as it provides a path for molten material and gases to flow to the burst vent in the case of thermal runway [351].

Other contributions show that FBGs, attached to the surface of free standing pouch or prismatic cells, provide meaningful and reliable data on the temperature and mechanical changes occurring as a function of the SOC during operation [352-354]. It is shown that FBGs exhibit $28 \%$ lower rise time compared to K-Type thermocouples, emphasizing their real-time monitoring capabilities [355]. Furthermore, it is suggested that FBGs are deployable within the jelly roll or even in the electrode coatings [336,356-359]. The mechanical and temperature changes during operation can be precisely monitored in this position. More comprehensive information on the application of FBGs are found in recently published reviews $[134,343]$.

Despite the promising results, several issues regarding the integration of FBGs in real applications should be considered. Few studies report that internally integrated optical fibers can cause significant mechanical damage to the electrodes and the separator during cell operation $[353,357,358]$. However, Raghavan et al. manage to successfully embed optical fibers during the assembly of $15 \mathrm{Ah}$ pouch cells with LG Chem's stack-and-fold bi-cell structure (see Figure 5d) and report stable cycling behavior even during stress test [336]. Moreover, the authors emphasize the applicability of their approach to EV batteries and project the costs up to the system level $[211,336]$. The additional cost is significant and thus regarded as an obstacle for the successful application of FBGs as cell integrated sensors.

The application of optical sensors for the characterization of Li-ion cells gained significant popularity in the scientific literature recently. Several publications of the same research group report measurements of the SOC of small laboratory cells. An optical fiber is embedded into the graphite anode to exploit the strong correlation of the graphite's reflectance in the near-infrared band and the anode's lithiation [212-214,360]. The authors suggest the application of in situ fiber evanescent wave spectroscopy (FEWS) as a costeffective, real-time, and robust method to monitor the optical changes of the graphite anode during operation. Their preliminary studies show that the capacity fade of the anode can be monitored in operando [361]. However, the presence of the optical fiber within the electrode can impair the lithiation in the vicinity of the sensor, impeding the Li-ion diffusion similar to defect-induced diffusion behavior changes [362]. Moreover, further studies on larger cells, where the SOC distribution within the jelly roll is more inhomogeneous, are essential for further consideration of FEWS for application in real battery systems.

In recent years, ultrasonic probing is gaining popularity as a non-invasive technique for characterization of commercial Li-ion cells. In ultrasonic measurements, an actuator emits a wave which propagates through the medium and a sensor measures the system's 
response to the excitation [343]. The measurements can be performed in reflection, throughplane, or in-plane modes, depending on the position of emitter and receiver. The higher the density of the sample, the higher the attenuation of the signal.

The performed studies reveal that SOC and SOH of Li-ion cells can be successfully estimated by ultrasonic probing. According to the literature, the mechanical changes that arise during operation, can be monitored by tracking the propagation speed as well as the amplitude of the signal. Davies et al. report that the propagation time and intensity of the through-thickness bulk waves can be used as predictors for $\mathrm{SOC}$ and $\mathrm{SOH}$ estimation. They use cell voltage, Time-of-Flight (TOF), and total signal amplitude metrics to train a simple machine learning model. According to the authors, an SOC prediction error of $\pm 1 \%$ is achieved [363].

Moreover, it is suggested that ultrasonic probing can be integrated into EVs and portable devices by using basic signal processing electronics and simple piezoelectric transducers [364]. Another use case is end-of-life assessment [365]. The majority of the studies use high frequency transducers, such as piezoelectric transducers. They offer an opportunity for smaller packaging, since the active element thickness is inversely proportional to ultrasonic wavelength [363]. Some authors suggest the operation at lower frequencies which enables the analysis of slow compressional waves [366].

Table 6 provides an overview of the research on ultrasonic probing for characterization of Li-ion batteries. The performed studies imply that ultrasonic probing of Li-ion cells is a promising technique for state assessment of different cell compositions. However, the majorities of the studies are performed with relatively small single pouch cells, and it is shown that the acoustic behavior becomes more complex as the number of pass-through layers grows [364]. Further studies on larger cells are necessary in order to access the applicability of ultrasonic sensors in EV battery systems. There is also a lack of studies on prismatic cells with wound jelly roll structure. In addition, concepts and examples for integration of the sensors on module and system level have not been yet reported.

Table 6. Application of ultrasonic probing for characterization of Li-ion cells in the literature. All cells feature graphite anodes. Abbreviations: pouch (po), cylindrical (cy).

\begin{tabular}{|c|c|c|c|c|}
\hline Cell Type & Capacity & Frequency & Operation Mode & Application \\
\hline LCO po & $210 \mathrm{mAh}$ & $2.25 \mathrm{MHz}$ & $\begin{array}{l}\text { temporally resolved pulse-receive } \\
\text { acoustic TOF measurements }\end{array}$ & $\begin{array}{l}\text { stress induced failure [367] } \\
\text { SOC/SOH }\end{array}$ \\
\hline LCO po & $200 \mathrm{mAh}$ & $2.25 \mathrm{MHz}$ & transmission mode & SOC/SOH estimation [363] \\
\hline LFP po & 1.1 Ah & n.a. & n.a. & n.a. \\
\hline LCO po & $1 \mathrm{Ah}$ & $5 \mathrm{MHz}$ & $\begin{array}{l}\text { pulse echo mode through } \\
\text { transmission mode }\end{array}$ & $\begin{array}{l}\text { detection of local } \\
\text { degradation [368] }\end{array}$ \\
\hline LCO po & $1.2 \mathrm{Ah}$ & $200 \mathrm{kHz}$ & transmission mode & SOC determination [366] \\
\hline LCO po & n.a. & $2.25 \mathrm{MHz}$ & pulse echo mode & SOC determination [364] \\
\hline NCA cy & n.a. & n.a. & through transmission mode & n.a. \\
\hline NMC po & $3.3 \mathrm{Ah}$ & n.a. & in-plane and through-plane modes & $\begin{array}{l}\text { mechanical structure during } \\
\text { high temperature abuse [369] }\end{array}$ \\
\hline NMC po & $3.65 \mathrm{Ah}$ & $100-200 \mathrm{kHz}$ & $\begin{array}{l}\text { acousto-ultrasonic guided waves } \\
\text { in-plane mode }\end{array}$ & $\begin{array}{l}\text { SOC determination intercalation } \\
\text { staging [370] }\end{array}$ \\
\hline NMC po & $36 \mathrm{Ah}$ & $200-600 \mathrm{kHz}$ & non-contact air-coupled & SOC determination [371] \\
\hline
\end{tabular}

\section{Reconfiguration in Intelligent Battery Systems}

Advanced monitoring with the necessary implementation of sensors in an IBS solves the challenge of unknown single cell states in automotive battery systems. However, even if the cell states are known and potentially weak cells are identified, the fixed interconnection 
of multiple battery cells in today's automotive battery systems prevents flexible intervention. The conceptualization of a multi-cell topology is defined in the battery design process and is permanently linked during production via rigid electrical connections. However, the operation of the entire system has to be restricted in such a way that the conditions are acceptable for each cell in the battery [372,373]. A promising technology to overcome these limitations is reconfiguration which has been widely discussed in the literature.

For this work, an IBS with reconfiguration functionality is called Reconfigurable Battery System (RBS), which is defined by the following capabilities in its minimum configuration (see also Figure 6):

- The smallest unit in an RBS can be a single cell or multiple cells combined. Since these cells do not necessarily have to be cells of the same type or even battery cells, the smallest unit is referred to as Energy Storage Unit (ESU) (Figure 6a). However, in the case of multiple cells, the flexibility of having influence on each individual energy storage decreases [374].

- An RBS is characterized by the fact that connections between the inherent ESUs can be changed during operation. As visualized in Figure 6b, an ESU can have three different states:

- $\quad$ An ESU can be switched out of the power path, while the power path is either further maintained after switching of the ESU (bypass state) and/or the switching of the ESUs disrupts the power path (idle state).

- An ESU is switched into the power path (active state).

- An important feature of RBSs is that these states are reversible and can be repeated multiple times during operation. Furthermore, such a system can be designed in a way that it contains multiple load paths and that an ESU is assignable to one or more of them. It is worth noting that an RBS needs to be able to change its configuration in real-time depending on system state [372,373].

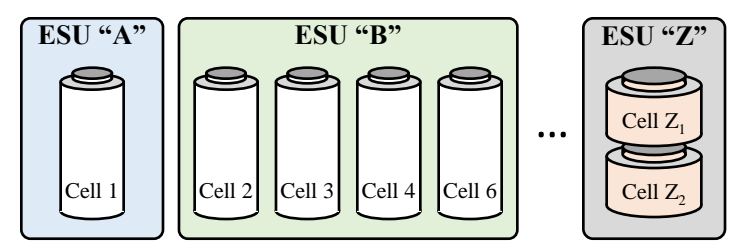

(a) Several possible ESUs as smallest unit in an RBS

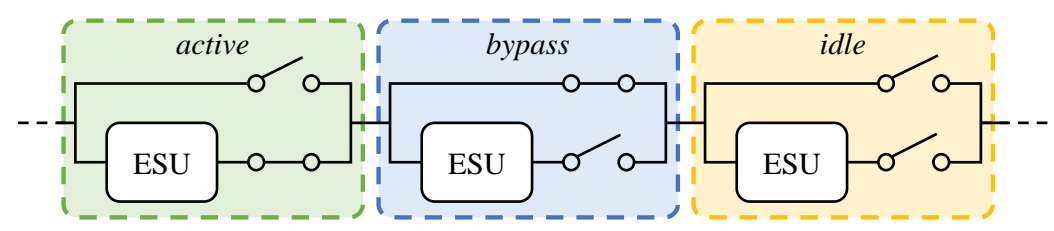

(b) Different reversible states of ESUs in a serial connected RBS with half bridge microtopology.

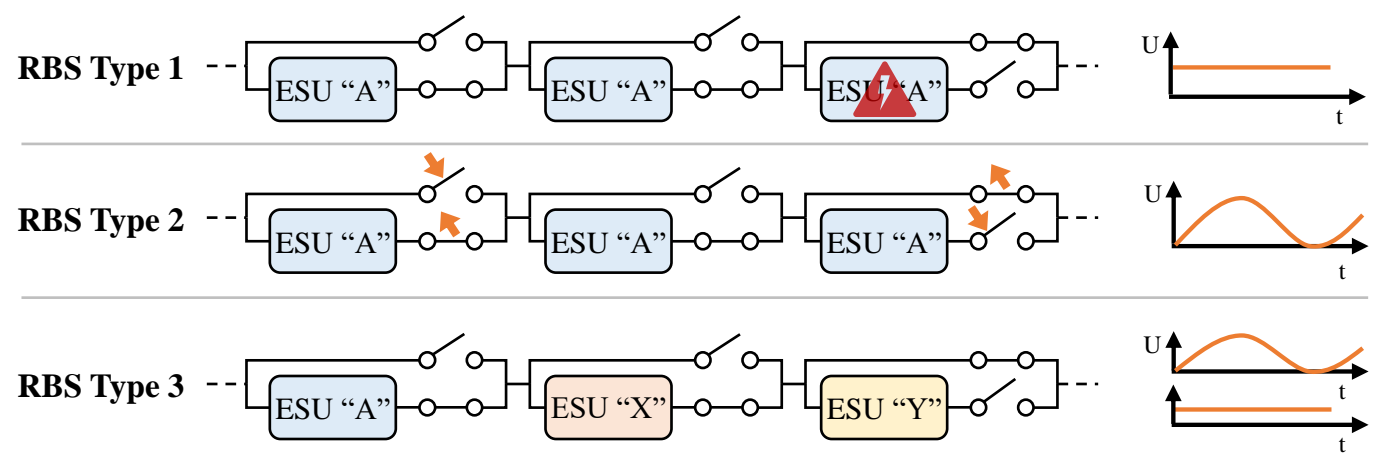

(c) Schematic representations of RBS Types 1-3.

Figure 6. (a) Visualization of possible ESUs including single cells, multiple cells, and different cell types. (b) A serial connected RBS with half bridge microtopology and all possible switching states. (c) Schematic representation of RBS Types 1-3 with the respective output voltage waveforms. 
Reconfiguration actively controls the energy flow through the battery system. As a consequence, new opportunities arise to influence system states in the battery system during operation and over lifetime. In addition, RBSs gain flexibility and scalability compared to conventional battery systems and increase their tolerance to failures of individual ESUs $[372,373,375,376]$. Different general expansion types of RBSs are differentiated in Figure $6 c$, since not all applications require an RBS with its maximum possible configuration or functionality. These general types are introduced by the example of a two-switch microtopology in serial configuration. The multitude of possibilities of different micro- and macrotopologies will be shown in the following sections:

RBS Type 1 uses reconfiguration to directly change the state of an individual ESU. The main goal of this expansion level is to change or adjust the SOC [377-380], the temperature, and the $\mathrm{SOH}$ of an individual ESU as well as to guarantee its operation in case of single ESU failure [379,381]. Reconfiguration is thus used to influence internal states so that the batteries performance matches a new one's for as long as possible. Since it has the same electrical output characteristics as a conventional battery but an extended lifetime, this technology can directly replace conventional battery systems in BEVs.

RBS Type 2 performs reconfiguration dynamically and continuously to achieve the desired condition to operate the load, resulting in AC output voltage wave-forms. This leads to a transfer of the power electronics into the battery system and thus a fusion of both components. In the literature, Type 2 is referred to as an Multilevel Inverter (MLI) [382]. Commonly, the system is arranged in multiple strings of ESUs to supply multi-phase AC. In an electric drive train, an IBS with MLI technology can directly operate an electrical machine without additional components.

RBS Type 3 are battery systems in the form of Type 1 or Type 2. They are used to operate different types of ESUs in one system. This type is called Hybrid Battery Storage System with the goal to combine ESUs e.g., with different energy storage capability or different power ratings [374]. With Type 3, an automobile manufacturer can, for example, replace defective cells in a battery system of an older costumer vehicle, even if the battery technology has changed in the meantime.

\subsection{Basic Functions}

The integration of switches into the battery system enables the BMS to directly control the operation of individual ESUs. In this way, it is possible to define which ESU actively contributes to the operation of the load. Furthermore, the load on the ESU has a significant influence on internal state variables of the ESU, such as the SOC, temperature, or aging. Reconfigurable IBSs can exploit this impact to increase the performance of the battery system. Thereby, the basic functions are mostly implemented with a single cell ESU, since balancing, detection, and handling of bad blocks is mainly dedicated to single cells.

\subsubsection{Bad Block Management}

As shown in Section 2.1, IBSs are equipped with the potential for extended fault diagnosis. In addition, with an RBS, a new control lever is established at the ESU level while conventional battery systems have no ability of error handling, except shutting down the entire system. In a first step, the switches of an RBS can be used to identify weak cells, since they are advantageously for active fault diagnosis [32,383,384]. Secondly, cells with unusual behavior can be removed from the load path at an early stage without having to interrupt the operation of the load [385]. Such procedures are called Bad-BlockManagement (BBM).

With a cell detected as abnormal, an RBS has the capability to reduce its effective operating time by dynamic bypassing or by switching off the cell permanently. The decisive factor is whether the state of a cell is defined as safe or unsafe for further operation [386]. Already, RBS Type 1 allows for an improved localization of faults within the battery 
system [32]. This is possible since RBSs can operate even if some ESUs are isolated or bypassed as long as the load demands can be fulfilled, and the battery system safety boundaries are taken into account. However, each isolated cell further limits the electrical performance of the battery system.

There are methods that try to tolerate ESU failures completely maintaining the electrical performance for a certain number of ESU failures [385]. For this purpose, redundant ESUs is kept available, which are not integrated into the operation or are cyclically exchanged with active ESUs $[387,388]$. This oversizing makes the system heavier, bulkier, costlier, and less efficient, since even bypassed cells generate ohmic losses while the maximum possible voltage is not used. RBSs usually has a large number of redundant switching states. However, the load can be operated normally even in the case of an error. If no redundant hardware is available, the full control is limited only by the faulty ESU $[384,389]$.

A further aspect of dynamic BBM comprising weak but safe cells is that the individual cell states are influenced by the BBM procedures [386]. Related to the thermal interactions, this aspect is discussed in Section 4.1.3.

For multi-phase systems of RBS Type 2, it is important to note that mostly symmetrical output voltage systems are needed, so that an ESU failure in one phase will affect the other phases in order to maintain the symmetry [389]. Thus, for AC voltage systems, more sophisticated methods are found in the literature, which enable increased fault tolerance without over-dimensioning. These methods do not require any additional hardware, since they are based purely on the control of the RBSs. These are procedures of the Neutral Point Shift (NPS) $[385,390,391]$. The NPS for fault tolerant operation tries to maximize the load voltage by adjusting the phase shifts of the output system bringing the load voltage systems amplitudes as close as possible to the desired original system [381,392-394].

\subsubsection{Balancing}

Deviations in SOC between ESUs can be caused by internal or external influences [373]. Internal imbalances are manufacturing variances, which are production-related differences in the capacities, internal resistances [395,396], and self-discharge rates [397]. Examples for external influences are the discharge via non-ideal parts of electronic circuits or safety components, leading to effects similar to self-discharge [396]. Both classes of influencing factors are subject to environmental influences such as temperature [396,397]. Since the ESUs in a conventional battery system is permanently connected to each other, the entire battery system can only be operated until the limits of the weakest cell are reached, even if other ESUs have not yet reached their cell-specific limits [372,376,396]. These differences can increase over the lifetime of the ESUs $[376,398,399]$. To prevent this, balancing procedures are implemented in battery systems.

Three types of balancing methods can be distinguished in conventional Li-ion battery systems: shunting, shuttling, and energy conversion. Shunting is often referred to as passive balancing [395] as the energy conversion is dissipative [400,401]. For the shuttling method and energy converter method, there are different topology architectures [395,402-404]. A classification can be made on the basis of energy flow either from single cell to pack, pack to single cell, or single cell to single cell. In general, the energetic poor passive balancing seems to have established itself in practice at present conventional battery system due to best cost-benefit ratio. There have been numerous reviews of these conventional methods concerning hardware and operating strategies in recent years [395,402-404]. In general, these methods can also be used in IBSs with an adequate operating strategy. At this point, we focus on a different balancing method, which shifts energy between the load and the ESU by using the reconfigurability of an RBS.

For RBS Type 1, where the output voltage must not change or should only change very slowly in relation to the discharge time, a compromise must be found between balancing functionality and output voltage quality. This is in order to create degrees of freedom for balancing on the one hand and on the other hand to minimize battery losses and meet the voltage requirements of the load. Manenti et al. [379] consider this condition as fulfilled if 
in large battery systems the weakest cell is taken out of the cell compound for adjusting SOC during operation. In this case, the previous redundant cell is switched back into the load path as a new one is isolated. Practical validation shows the feasibility of this method.

Bouchhima et al. [405] utilize dynamic programming to solve a finite horizon nonlinear optimal control problem using network modeling in order to find optimal distribution of balancing impact. The algorithm decides how much balancing is currently justified to achieve minimal power losses, if future charges are comparable to historic power distributions. The authors show that the model is robust against inaccurate power prediction. The approach is advanced and designed for online application, but the algorithm becomes computationally complex when applied to larger battery systems.

In contrast to the systems described above, RBS Type 2 permanently changes its output voltage. The discharge time is orders of magnitude greater than the cycle times of the periodic voltage changes. Thus, charge balancing methods are an integral component, since these systems use reconfiguration for the adjustment of the output voltage $[406,407]$ with the charge withdrawal being cell-individual. It is irrelevant for the output voltage for which ESUs are cascaded to form the output voltage as long as the sum of the voltages produces an acceptable output voltage level. This results in intrinsic degrees of freedom for balancing.

Focusing on RBS Type 2, a simple balancing-algorithm is implemented in $[380,387,408,409]$. Thereby, the assignments of the ESUs to the voltage units are periodically rotated. Therefore, the average load times are theoretically identical for all ESUs over the entire operation time. The method is statically not able to compensate for deviations of ESU or for example initial SOC deviations.

A comparable method is used by Babaei et al. [410], where a shift angle optimization for energy equalization is proposed. However, this method only considers the charge inequalities that are introduced due to the multi-phase functionality. The actual load is taken into account; however, classical reasons for charge differences like self-discharge and capacity differences are not considered.

Chang et al. [411] present a phase-shift carrier modulation for an active cell voltage equalization, where a closed-loop controller adjusts the phase shift angles according to voltage inequalities. Nevertheless, due to the carrier-based method, only voltage and not a SOC-based balancing can be performed [411,412]. Due to aging, this can lead to short-term load balancing processes reducing the effectiveness of the procedure.

Methods using sorting algorithms in combination with an RBS are often used to achieve equally charged battery systems [413-415]. Their aim is to assign higher charged cells to lower voltage levels, since these levels are operated longer resulting from the typically sinusoidal-shaped waveform of the output voltages. The sorting algorithm evaluates voltages or SOC and adopts evolving differences in a closed loop overall battery control strategy during operation.

Goetz et al. [416] introduce balancing strategies for topologies with parallel connecting capability. For this kind of topology, cells are not switched in idle, but the individual cell load is reduced through parallelization of cells. Therefore, besides minimization of losses, the controller keeps track of the maximum time each cell was in a parallel configuration.

Especially for RBS Type 2 in star-configured macrotopology, three or more phase strings are set up. Usually, each string consists of a number of serial cascaded ESUs. To operate a three-phase load, all three phases are needed. Therefore, a balancing between the phases has to be considered to prevent early stopping due to under- or overcharge of one string while the other two strings are still operable [406,407]. To ensure inter-phase balancing for a converter topology, an asymmetric power distribution over the three phases of a three-phase converter must be established. Therefore, the overall performance must correspond to the required performance. Hardware solutions are one option, but they are bulky and expensive. In the literature, multiple software approaches are investigated [417-419].

Balancing by reconfiguration is a convenient way for RBS to solve balancing issues in a simple and flexible way. It is superior to conventional balancing systems because 
it is software-based and no additional hardware is required. Furthermore, it is truly lossless and adjustable to different balancing needs over lifetime. However, balancing by reconfiguration is only possible in operation with load currents.

Even though balancing by reconfiguration is generally based on the same principle for all RBS types, a distinction must be made in the application between RBS Type 1, Type 2, and Type 3. For RBS Type 1, balancing is in general in direct conflict with energy efficiency for all balancing strategies, since the output voltage is reduced when cells are switched out of the load path. However, in the opinion of the authors, the simple redundant cell approach is a sufficient method for most applications and can also be used with a rule-based on/off strategy to optimize efficiency based on different load situations.

Advanced balancing approaches do theoretically have better efficiency by finding optimal solutions, but this does not seem to justify the computational effort in terms of energy and hardware costs. These algorithms constantly try to exploit off-peak periods. However, these are abundant in the operation of electric cars and therefore do not need to be used particularly efficiently.

For RBS Type 2, balancing becomes an integral part of the system but is intrinsic and truly lossless, as reconfiguration is part of generating AC output voltages. A reasonable approach might consider equal load methods to reduce balancing effort by a static balancing rule, but do not consider ESU differences. Additional methods should be used to account for random ESU state deviations based on the actual system states. Since these effects are small, the balancing methods should again be as simple as possible and balance the different SOCs of the ESUs in a controlled manner. In RBS Type 3, the inherent balancing properties of RBS are exploited to combine heterogeneous ESUs into a single battery system. Depending on the degree of inhomogeneity, more advanced balancing procedures may be required to manage the balancing effort.

\subsubsection{Wear Leveling}

The term balancing is usually limited to SOC balancing. However, the given methods can also be extended to other ESU states. In the literature, there is also an attempt to adjust the aging behavior of ESUs in a battery systems [376,420] and often the temperature is included in this context due to its relation to aging and safety [421,422]. Operating every cell in a module/system the temperature depends in general on the cell specific heat generation of various cells and their position regarding the cooling system. If it is possible to bypass cells in the system, the local heat generation of the bypassed cell is approximately zero, while the cell current of the remaining active cells increases automatically to meet the performance requirement. With knowledge of the resulting thermal behavior regarding bypassing a cell or a parallel string, it is possible to implement intelligent algorithms that perform balancing by switching not only for the thermal state. Furthermore, the focus of wear leveling is to optimize multi-dimensional inhomogeneities e.g., of SOC, current, temperature, and aging [2] comprehensively within the system.

Bouchhima et al. [423] evaluate the possible impact of an RBS on the main driver for aging of Li-ion batteries including cell temperature, OCV, Depth of Discharge (DOD), and charge throughput. Since most of these parameters are influenceable by reconfiguration, an aging model is introduced. Based on the calendric and cyclic factors, a significant enhancement on the battery lifetime and energy throughput over lifetime with an RBS is achieved. Moreover, it is shown that cell inhomogeneity decreases with RBS operation. Kleiner et al. [424] introduced an adaptive balancing algorithm with the focus on thermal homogeneity implemented on a reconfigurable module hardware. Thereby, the authors pointed out the resulting thermal effects of the current increase as well as the interactions of temperature and SOC homogeneity related to RBS procedures. Atlaf et al. [421] consider the simultaneous balancing of SOC and temperature. The close dependence of the two quantities on the current flow is modeled, and an active balancing of SOC and temperature is illustrated in a suitable control strategy. However, the use of the predictive control framework solves a rather complex control problem which is computationally expensive. This 
fact is addressed in [425], where model predictive control and an orthogonal decomposition into two problems are implemented. Thereby, the temperature and the SOC-balancing are solved with limited knowledge on future load information. The presented methods are strongly based on a suitable aging model. Li et al. [420] propose a method that focuses on the change of the SOC while the cells are loaded with the same power. This is to detect deviations in the capacity and the internal resistances. The method has been designed on an MLI and considers circulating currents.

Generally, multi-objective wear leveling approaches become maximum attractive with RBS in combination with advanced monitoring procedures. Not only dual-objective balancing of SOC and temperature but further important states or influences on the wear level, such as $\mathrm{SOH}$ and fault states, can be integrated. The goal is to find the optimal load scheduling for the specific system in combination with the application. However, the influence of switching procedures on aging or efficiency aspects as mentioned in Section 5.4 should not be neglected.

\subsection{Switching Topologies}

The functionality of reconfiguring ESUs is nowadays technically implemented by the design of a connection network between the ESU and the selective insertion of switching elements into the network trajectories. The interconnection of the ESU and the circuit network is also referred to as a module. In such systems, these modules are reconfigurable with each other, since the switching elements are able to activate and deactivate electrical connections [375,426,427].

In the following, only cascaded topologies are considered in which each ESU can be assigned to a defined structure of switching elements and connection paths. Therefore, known MLI classes such as Flying Capacitor (FC) [428] and Neutral Point Clamped (NPC) topologies $[429,430]$ are not considered. The structure of the electrical paths within a module, which connects a number of module terminals directly or via switching units with the terminals of the ESU, is referred to as microtopology [374]. By multiple use of this microtopology in an electrically cascaded manner, a larger storage system can be created which has a higher voltage and/or current carrying capacity. The result of the cascading process is called macrotopology, where the microtopologies can be switched in series, parallel, or both [416,431,432]. Possible topology implementations for RBSs are therefore not necessarily of the same quality, since they can vary in the number of inherent switching devices or in the way they are interconnected. For this reason, a large part of the recent research dealing with RBSs is focused on the development of innovative and most appropriate structures. Topologies in general differ in the number of inherent switches, their arrangement as well as the presence of possible connections between the ESUs. In addition, they are distinguished depending on the number of immanent electrical ESUs and their resulting voltage value.

\subsubsection{Microtopology}

In Table 7, various different microtopologies are listed. They mainly differ in their respective functional range. Simple structures, such as the half bridge proposed by Darco et al. [413], provide the basic functions for an RBS in serial configuration. The half bridge design is one of the most frequently used topologies for RBSs today $[375,381,408$, 413,433-443]. The main advantage of this topology is its low complexity. For this reason, the circuit is used in applications ranging from pure RBS Type 1 to voltage adjustable batteries like MLIs (RBS Type 2 and 3). They are able to disconnect any ESU from the load path without interrupting the supply to the load $[375,434]$. The switching state of each module is independent of the switching states of the other modules [375,413,433,434,438,444-446]. Due to the great importance of the half bridge topology, investigations have been made to further improve this circuit by minimizing the number of switches in the load path, while maintaining the same functionality. Choi et al. proposed in [447] a Prior half bridge topology. It is characterized by having one switch in the load path for the case of an 
active ESU, whereby no switch is in current path if the ESU is bypassed. However, this drastic reduction in switches causes dependencies that inhibit ESU-independent bypassing. The half bridge topology is suitable for most RBSs. Drive applications require sinusoidal voltages and currents that contain positive and negative voltage segments. The half bridge misses the function of polarity reversal at the module terminals though.

Table 7. Overview of microtopologies from the literature with the number of submodules $N$ and the instantaneous output level $n$. All associated macrotopologies achieve the full $2 N+1$ output levels but may need a polarity reversal circuit to do so. The number of switches is counted with respect to the macrotopology, which includes the potential polarity reversal circuit. Abbreviations: serial switches (SS), total switches (TS), polarity reversal (PR), serial bypass (SB), parallelization (PA), external (ext), internal (int), Common Emitter Bidirectional Switched Based MLI (CEBS).

\begin{tabular}{|c|c|c|c|c|c|}
\hline Microtopology & SS & TS & PR & SB & PA \\
\hline $\begin{array}{l}\text { Half Bridge } \\
{[375,381,408,413,433-443]}\end{array}$ & $N+2$ & $2 N+4$ & ext & yes & no \\
\hline $\begin{array}{l}\text { Prior Half Bridge } \\
\text { [447] }\end{array}$ & $\begin{array}{c}n+2 \\
\text { Level } 0: 2 \\
\text { Level } N: N+1\end{array}$ & $2 N+2$ & ext & no & no \\
\hline $\begin{array}{l}\text { Cross-Switched } \\
\text { [448] }\end{array}$ & $N+1$ & $2 N+2$ & int & no & no \\
\hline $\begin{array}{l}\text { Cascaded Cross-Switched } \\
\text { [449] }\end{array}$ & $\frac{3}{2} N$ & $3 N$ & int & yes & no \\
\hline $\begin{array}{l}\text { CEBS } \\
{[450]}\end{array}$ & 4 & $4 N+4$ & int & no & no \\
\hline $\begin{array}{l}\text { Full Bridge } \\
{[380,385,407,410,418,419,} \\
435,451-456]\end{array}$ & $2 N$ & $4 N$ & int & yes & no \\
\hline $\begin{array}{l}\text { ECIN } \\
{[457]}\end{array}$ & $\approx N+1$ & $4 N+2$ & int & yes & no \\
\hline $\begin{array}{l}\text { Marx } \\
{[458-461]}\end{array}$ & $\leq N+2$ & $3 N+4$ & ext & yes & yes \\
\hline $\begin{array}{l}\text { MMSPC } \\
{[416,431,435]}\end{array}$ & $\leq N$ & $8 N$ & int & yes & yes \\
\hline $\begin{array}{l}\text { M2B } \\
{[455,462,463]}\end{array}$ & $\leq 2 N$ & $9 N$ & int & yes & yes \\
\hline $\begin{array}{l}\text { PECIN } \\
{[464]}\end{array}$ & $\leq N+1$ & $6 N+2$ & int & yes & yes \\
\hline
\end{tabular}


In $[437,440,465]$, this problem is solved on the macrotopology level by connecting an external polarity unit in the form of a full bridge at the terminals of the entire cascaded string composed of the half bridge circuits.

In order to avoid this external device or a reduction of the maximum output level of the battery system, further research was carried out to create this functionality on a microtopology level. In [448], Kangarlu et al. present a Cross-Switched MLI having only two switches per ESU. It has the capability to generate positive and negative output voltage on the phase terminals [466,467]. Resulting from the absence of individual bypass capability and in order to increase the number of possible voltage levels a Cascaded Cross-Switched MLI is introduced by Sabahi et al. [449]. Babaei et al. [450] present a comparable simple but energetically optimized topology. With the Common Emitter Bidirectional Switched Based MLI, the number of switches in the load path is always four regardless of how many of these modules are serially connected. Even for a small number of ESUs, the internal resistance is therefore similar to a conventional battery system [468]. However, the small number of switching elements leads to a reduced flexibility in reconfiguration; therefore, e.g., an ESU individual bypass is not possible. Ebrahimi et al. [469] present a unipolar version of the circuit. This variant saves components, whereas the missing bi-directionality is created by a downstream full bridge.

One of the most frequently investigated microtopologies is the full bridge topology, also named Cascaded H-Bridge (CHB) [380,385,407,410,418,419,435,451-456]. It offers the same functionality as the half bridge, but the polarity inversion is directly integrated into the microtopology [470]. Therefore, it is able to switch each individual ESU positively or negatively into the load path. This offers a larger number of voltage levels but doubles the switches in the load path for both active and bypass cases [470]. Thus, topologies such as the CHB and the following more sophisticated topologies are mostly addressed if MLI capability is requested. In the case of a Type 1 RBS, the half bridge circuit as in [375] is typically preferred.

Terbrack et al. [457] have developed the so-called Enhanced Commutation Integrated Nested (ECIN) topology. It reduces the number of switches in the load path for active ESUs to one. Despite the lower internal resistance, it uses only four switching elements in its microtopology, as the CHB. In addition to the functionality of voltage reversal, there is a large number of topologies that extend the half bridge topology by the functionality of parallel connection of ESUs $[458,460,461]$. An important field of application is the reduction of the internal resistance of the battery system as well as the active switches in the load path by this technology. In particular, this is feasible if the desired output voltage of the RBS is lower than its maximum generable voltage value. In this case, some ESUs are temporary out of use [457].

A side effect of the topologies, which can connect cells not only in series but also in parallel, is that the usual two connection terminals are no longer sufficient for one module. For these microtopologies, two connection points must be provided to the previous and also to the subsequent ESU, so that a parallel connection is feasible. In technical realization, there are topologies which require four terminals for the microtopology and topologies which manage with three terminals. Doing so, the third terminal must be connected to the preceding and subsequent ESU [374,416,429,431,471]. Especially in MLIs, where the output voltage is periodically changed, advantages can be achieved with parallel reconfiguration [472]. Compared to the CHB topology, however, e.g., the Marx topology presented in $[458,459]$ does not have the capability of direct voltage inversion. The topology is further investigated and called BM3 by Helling et al. [460] while Kim et al. [461] call it DESA. These topologies also have to use external commutation units for MLI operation guaranteeing positive and negative output voltages. The so-called MMSPC topology presented in $[416,431,435]$ combines the functions of polarity inversion, parallelization, and individual control of the ESUs in one topology. Therefore, it integrates the entire range of functionality in one circuit. The topology consists out of two full bridges, containing at least eight switches. In the purely serial case, there is only one switch in the load path for both 
active and bypass. This number can be effectively reduced by parallel connection of ESUs. Helling et al. [462] present an M2B MLI with the advanced functionality of parallelization of cells. Thereby, parallel groups of cells can be formed even across defective ESUs by inserting a further switch in series to each ESU [462]. However, for this reason, the number of switches in the load path increases significantly, which leads to higher conduction losses. In general, MMSPC and M2B are sophisticated but hardware intensive topologies. Terbrack et al. [464] have implemented the Parallel Enhanced Commutation Integrated Nested (PECIN) topology design. It incorporates all the functionality of the MMSPC, but with reduced hardware effort.

The microtopology already defines important features of the overall RBS. The topologies presented attempt to minimize electrical components while maximizing functionality. Especially for EVs, the way the microtopology is derived from the overall topology is important to get few and equal parts for assembly. Since cost is a major driver in industrialization, the goal is to keep electrical equipment to a minimum. From a technical point of view, the efficiency of the RBS benefits from the lowest internal resistance of the microtopology. For these reasons, the half bridge topology is most appropriate for RBS Type 1. More sophisticated approaches are discussed for RBS Type 2, since polarity commutation is an integral part of $\mathrm{AC}$ voltage generation. Topologies with external or intrinsic commutation are presented in the literature. The half bridge or the MARX Inverter in combination with an external full bridge are suitable candidates with low internal resistance. However, topologies with intrinsic commutation do not require any additional external hardware component. Since cost is a major consideration, the system designer needs to be clear about the functionality required of the RBS system, as generally more functionality comes with more hardware expense. The ECIN topology has very low internal resistance compared to its number of components. If a more sophisticated approach is appropriate, PECIN has slightly more hardware effort, but the additional ability to reduce internal resistance by paralleling the battery cells, which increases the efficiency.

\subsubsection{Macrotopology}

There are different possibilities to cascade the microtopologies to a larger RBS. In most cases, only one type of microtopology is cascaded to a specific macro-version. However, it is possible to integrate different types of microtopologies in a macrotopology as it was executed, for example, by Hinago et al. [432] or by adding a downstream full bridge as polarity unit as in $[437,440,465]$. The combination of different topologies is in the literature referred to as hybrid MLI $[382,467,473]$. The macrotopology has a great influence on the function of the entire battery system and significantly determines its performance and behavior. Therefore, the macrotopology is strongly dependent on the application. An overview of common macrotopologies for reconfigurable IBSs is given as schematic visualization in Figure 7.

A straightforward connection of microtopologies is based on a conventional battery system cell connection either in serial, parallel, or serial and parallel configuration (Figure 7a-d). Typically, RBS Type 1 often do have these configurations [375,379,437,441,442]. For MLI applications, the star configuration (Figure 7f) is utilized in most cases [470,474], since it delivers the highest output voltage for a given number of ESUs. A delta configuration of submodules (Figure 7g) is proposed by Helling et al. [374] in order to improve the reliability of electric cars at a fault in one electrical phase. Since the delta configuration is fault-tolerant [374], a faulty string leads to the presented reduced string configuration which only uses two thirds of the otherwise needed ESUs of the delta configuration. Especially for BEV, a common DC-link is important to provide energy not only for the main drive, but also for electric auxiliary consumers.

The star, delta, and reduced string configurations (Figure 7f-h) do not provide a DClink [374,416]. However, the Modular Multilevel Converter (MMC) topology (Figure 7e) provides a DC-link. Therefore, the MMC is a well-investigated inverter design. Characteristic for the MMC is that circulating currents are emerging, which on the one hand 
are undesirable and can be reduced by control strategies or further included arm inductors [475]. On the other hand, the phase arms are balanced to each other automatically $[433,434,445,446]$. The MMC is mostly used with half bridge submodules, but there are also variants that for example use full bridge and Clamped-Double microtopologies $[467,476]$. Nonetheless, in MLI applications, the MMC provides only a quarter of the entire DC-link voltage on the AC-connections, which is a considerable disadvantage for drive applications $[431,433,434,445,472]$.

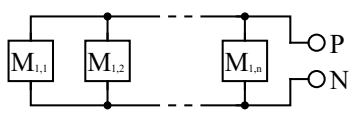

$\mathrm{a}$

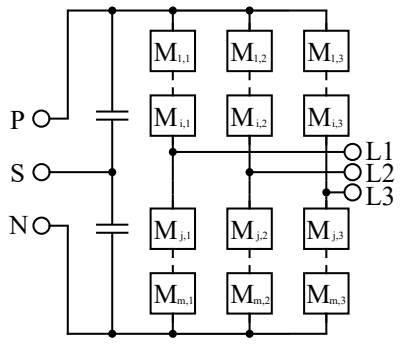

$\mathrm{e}$

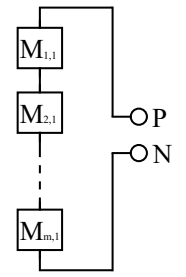

b

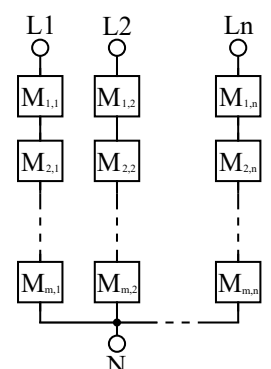

$\mathrm{f}$

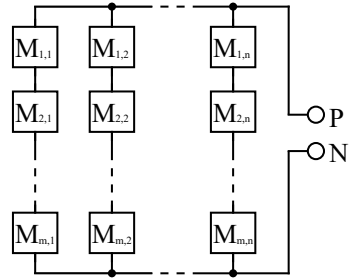

c

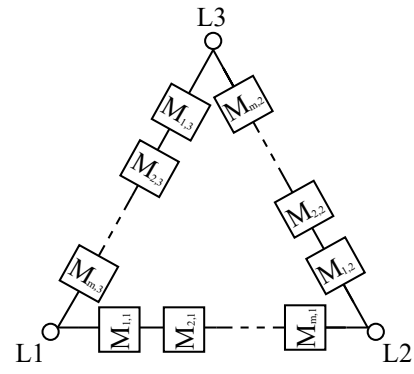

$\mathrm{g}$

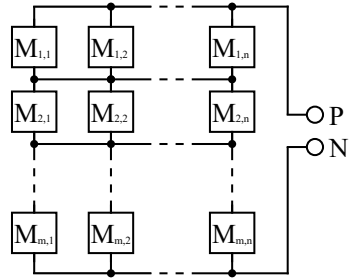

d

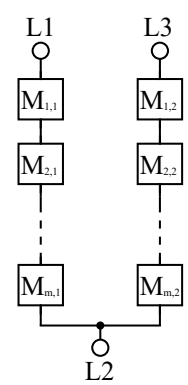

$\mathrm{h}$

Figure 7. Schematic visualization of different macrotopologies for reconfigurable IBSs: (a) parallel circuit, (b) serial circuit, (c) serial parallel configuration, (d) parallel serial configuration, (e) MMC topology, (f) star configuration, (g) delta configuration, (h) reduced string configuration.

Terbrack et al. [457] introduce a new approach to achieve a DC-link with as few components as possible by integrating additional voltage taps into an MLI battery system. Therefore, two or more MLI systems arise and are integrated into one battery system. They independently supply individual adjustable output voltage systems but have access to shared ESUs. An externally excited synchronous machine is operated with its three-phase stator system and a controllable DC-voltage for the rotor system. In addition, there are approaches with an RBS to generate several different output voltages simultaneously in order to be able to operate different loads. Helling et al. [462] integrate so-called low voltage power supply units in the phase arms to tap additional consumers. A further method is presented by Kuder et al. [455], where two electrical machines are operated simultaneously with the same MLI. However, this concept exhibits a higher control complexity, without providing benefits in the hardware implementation.

In general, the most commonly used topology for conventional battery systems is the parallel-serial configuration, which is directly transferrable to RBS Type 1. For RBS Type 2, multi-phase approaches need to be considered. The star topology is the one most considered due to the maximum output voltage and its simple structure. However, a major drawback for RBS Type 2 is the missing DC-Link circuit for auxiliary devices. There are approaches to provide a DC-Link, but they increase the complexity of the system. Furthermore, the MMC topology that provides a DC-Link is inefficient in EV applications due to a low AC output voltage.

\subsection{Multilevel Inverter}

As already briefly introduced, an MLI is the most common form of RBS Type 2. This kind of inverter is a frequently investigated topic in the past few years and a still growing 
research area. Its functional principle is based on the generation of AC output voltages formed by stepped wave-forms, whereby typically each step is built by one ESU.

Depending on the respective control strategy, an individual decision is made for each ESU on how it contributes to providing a desired output signal of the entire battery system as efficiently as possible. Due to this feature of composing reference voltages by small voltage steps in form of the ESU, the resulting output voltages correspond to the required signal in a very precise way. In particular, for the application in EVs, this system offers its potential in the partial load operation, where conventional power electronics can only switch the entire battery voltage [435,477]. This results in better performance of the MLI in the entire speed-torque range. Besides this feature, other advantages such as lower EMI and consequently smaller filter effort, the application of power semiconductors with smaller breakdown voltage, lower proportion of harmonics in the output signals, and smaller Total Harmonic Distortion (THD) can come along with this technology. The typical field of application is the medium- and high power and voltage range [426,429-431,433,434,444,445,448,468,472,478].

However, there are also some challenges that need to be overcome, such as the need for a large number of switching devices $[448,468]$ and the increasing computational complexity of the control strategy as the number of ESUs increases. For this reason, the quality of the topology and the control methods are decisive levers for the best possible utilization of an MLI. Using the example of the powertrain of a BEV (Figure 8), the modulation scheme for the switches is included in the operating strategy.

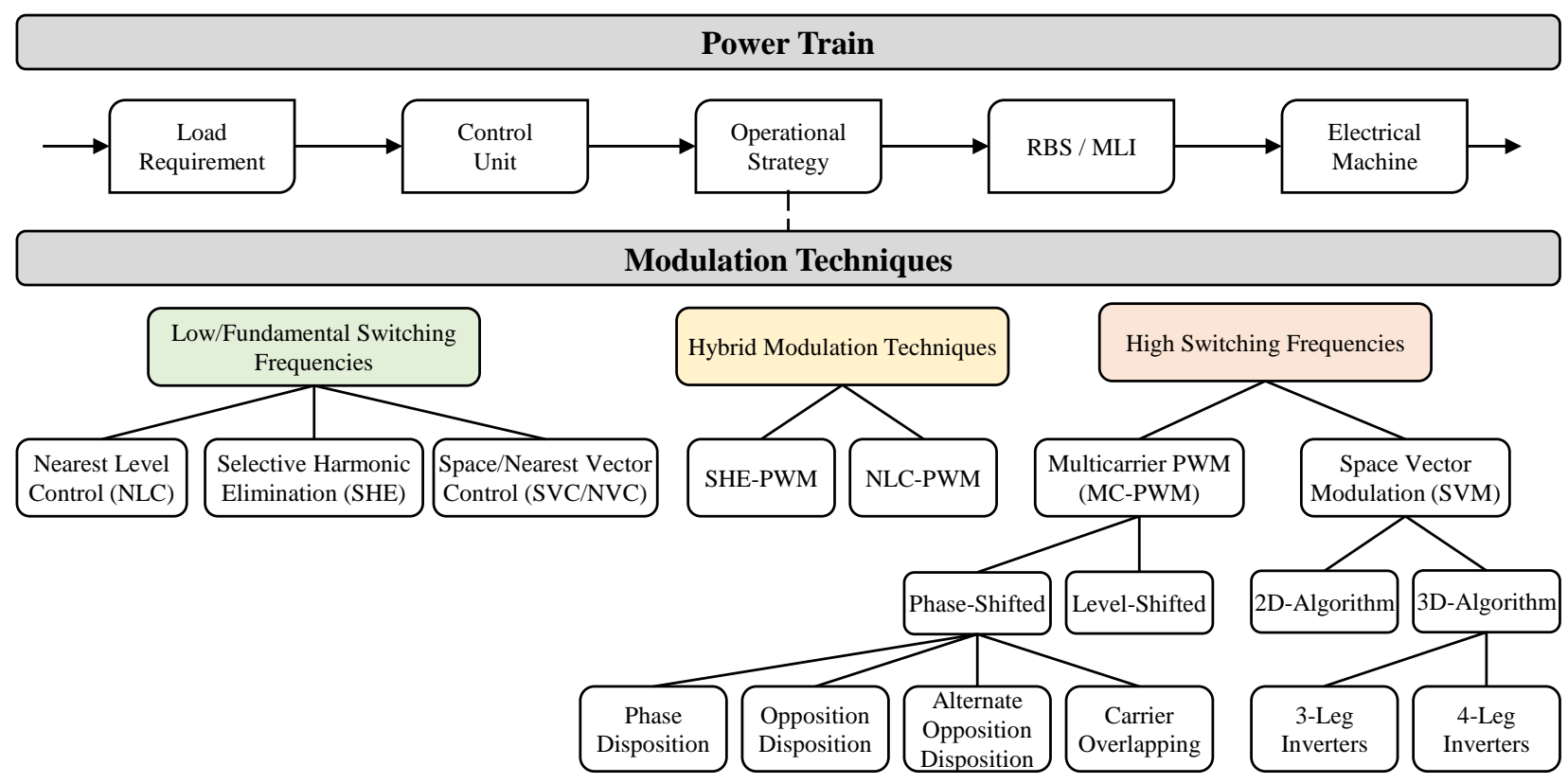

Figure 8. Schematic flow chart of the power train and cluster of modulation techniques.

In the literature, a large number of modulation schemes exists [444,478,479]. On the one hand, they can be classified either depending on the domain, in which the generation of the demanded voltage level for a specific time interval takes place [444]. Thereby, it can be distinguished between the time-domain and the state-space vector domain. On the other hand, the strategies can be clustered with regard to the underlying switching frequencies $[444,480]$. An allocation in dependence to the respectively switching frequency is shown in Figure 8.

The choice of the right modulation strategy largely depends on the environmental conditions of the system. Some of the most important selection factors are the requirements for overall system efficiency, losses caused by the inherent switching elements as well as the harmonic content of the inverter output signals. For intelligent battery systems, all modulation schemes are conceivable at first sight. However, since e.g., the application in 
BEV requires a high-dynamically system with real-time characteristic, the computational effort of the chosen variant should be low. Due to the typical high number of included ESUs, attention should also be paid to the switching frequency of all inherent switching devices. With increasing switching frequency, the total switching losses usually increase by the factor of the number of ESUs. One comparatively simple method to control the switching signals of an MLI is the Nearest Level Control (NLC) [481]. In this time-based switching strategy, the output voltage level is chosen that is closest to the desired reference vector $\left(V_{\text {ref }}\right)$ [427]. Only one switching operation per voltage level is performed, and thus the utilized switching frequency is in the fundamental range. Consequently, the entire switching losses are kept low. No carrier signals are required to generate the control signal for each individual ESU in the MLI structure. The NLC variant is flexible and stands out for an easy digital implementation [20,482], which makes it attractive compared to other strategies. This fact becomes more important with increasing number of ESUs or rather levels. As mentioned in [481], the NLC scheme is in fact no modulation technique, since no averaging processes take place. In general, all switching strategies are summarized under the term of modulation techniques though $[426,431,444]$. With the NLC method, suppression of harmonics is not feasible [483] and thus the harmonic content cannot be minimized specifically. As a consequence, the performance suffers [481]. This fact gets even more obvious for the case of a lower number of inherent ESUs in the entire system, since the proportion of harmonics is comparatively higher [427].

This problem is addressed with Selective Harmonic Elimination (SHE) strategies, which, however, still use low switching frequencies [426,427,444,481]. With the elimination feature, the harmonic content decreases while low switching frequencies result in low switching losses. Kamaldeep et al. [427] investigated the comparison of the NLC- and the SHE-method for a seven level MLI. The results show that a smaller THD value of harmonics up to the 5th order is achieved when applying the SHE instead of the NLC. For SHE, the Fourier coefficients of the expected reference signal are examined in an offline procedure before the actual operation. From this system of equations, the associated switching angles $\left(\alpha_{1}, \alpha_{2}, \alpha_{3}, \ldots, \alpha_{n}\right)$ are determined [481]. Special numerical methods or optimization algorithms such as Particle Swarm Optimization (PSO) or GA are utilized to calculate the most suitable angles [454,484]. With the help of lookup-tables, the pre-calculated switching angles are applied in operation and interpolated with respect to the actual operating point [444]. However, the complexity of these elimination procedures due to the underlying numerical solving processes increases with the number of MLI levels. Thus, the application of offline-based SHE is not the most appropriate one, especially for systems with high-dynamic demands [481]. In addition, the required storage of the lookup-tables complicates the usage in real-time applications [444] and diminishes its implementation in low cost microprocessors. Furthermore, there is no control over the higher order harmonics that are not specifically suppressed with this method. As mentioned by Rodriguez et al. [481], they can become higher, since the energy of the eliminated harmonics is allocated to the other ones.

Therefore, another approach called SHE-PWM is developed that has more than only one switching operation on a level. Li et al. [485] introduce SHE-PWM as systematic control strategy for cascaded MLI. Since those methods have similar outcome as high-frequency based strategies described in the following, the elimination of low order harmonics seems to be very expensive, especially regarding computational as well as storage effort.

A less complicated method is the so-called Space Vector Control (SVC), which is better suited for dynamical applications and does not need any lookup-tables like multiple SHE or SHE-PWM techniques [481]. It can be seen as a counterpart to the time-domainbased NLC-method. Like the NLC, its principle follows a fundamental switching frequency, but depends on the closest voltage vectors in the space vector plane [486]. One drawback of the SVC is that it suffers from the non-existent elimination of harmonics resulting in higher THD and performance losses as NLC does [444,481]. However, the results of Rodrigues et al. [486] show that the SVC method performs better regarding the voltage THD than a low-frequency switching Multi-Carrier (MC)-based PWM method. 
To combine the benefits of both methods, SVC often is equipped with overlaying PWM resulting in the Space Vector Modulation (SVM). Its procedure is similar to the SVC and thus takes place in the space vector-domain but has a comparably high switching frequency $[444,481]$. Contrary to the SVC, the reference vector is generated by means of averaging processes. Therefore, the neighboring space vectors of the reference voltage are switched sequentially depending on the underlying switching frequency. SVM as well as SVC directly deal with all three (or more) phases compared to NLC or SHE where the voltages of each phase are respectively controlled [426,482]. Thus, they superimpose in terms of criteria such as performance, simple implementation, and maximum transfer ratio [426].

As mentioned by Franquelo et al. [444], a high number of SVM methods have been proposed, but most of them are developed only for a certain number of inherent ESUs or output levels. Attique et al. [487] summarize five different SVM strategies, also named Space Vector Pulse Width Modulation (SVPWM), all based on a differing reference system. All strategies are usable for any number of voltage levels as performed by Prasad et al. [488]. In addition, the strategies are less complex compared to conventional SVM as the ordinary calculations have been transferred to simplified line voltage forms.

In recent years, many improvements of a conventional SVM technique are performed, which becomes more complicated with an increasing number of output levels [487]. An overview of recent development of simplifying and improving SVM methods is given by Franquelo et al. [489]. The listed schemes thereby avoid the use of trigonometric functions or other complex calculations by utilizing drastically simplified computations [489]. Moreover, no lookup-tables are used [444]. Keeping this in mind, the more advanced SVM strategies are promising candidates, especially because they can regulate more phases simultaneously and keep the computational costs low.

Other commonly used modulation strategies are the general PWM techniques. Although the SVM uses averaging and, thus, modulation, processes, the difference compared to the PWM-based schemes is that the latter are based in the time-domain and do have multiple carrier signals. In contrast to the determination of the switching times in the space vector plane as a function of the position of the reference vector, with PWMstrategies [426,481], a time-based comparison between the reference signal and specific carrier signals determines the switching times. In most cases, Sinusoidal Pulse Width Modulation (SPWM) is utilized as in [446]. The underlying carrier signals often have triangular waveforms but can also be saw-tooth-shaped [490]. The number of carrier signals in SPWM is normally equal to the number of inherent ESUs. During operation, each carrier signal is compared to the reference signal. The resulting signals are pulse-sequences that are allocated to the corresponding switching elements of the ESUs.

Although the SPWM strategy is simple and the switching frequency is adaptable to the use case, the generation of multiple carrier signals must be considered and can be very challenging. In particular, with an increasing number of ESUs, the number of carrier signals that are needed also increases significantly, which is why we consider SVM to be more appropriate.

Based on the descriptions above, the most promising candidates of modulation schemes in intelligent battery systems are NLC and SVM methods. In both cases, complex equation systems do not have to be solved to determine specific switching angles as in the SHE methods. Although certain harmonics can be eliminated, the latter technique often employs the use of lookup-tables. The NLC method as well as the SVM variant are characterized by simple implementation and outstanding dynamic performance [20,426]. In [482], an NLC-PWM (also called Nearest Level Modulation) is used having a higher switching frequency than the traditional NLC method. Thus, the NLC strategy can also be applied with higher frequencies. This reduces the THD of the output signals and the harmonic components are shifted to higher orders. Consequently, its disadvantage decreases compared to SHE. To optimize both methods depending on the actual operation point, the switching frequency can be varied for both strategies and adapted to the current operating range. Hence, the switching frequency is always as high as needed and not overdesigned. 


\subsection{Hybrid Battery Storage System}

Battery systems containing individual ESUs that differ significantly in electrical, mechanical or thermal properties are often refereed to as Hybrid Battery Storage System (HBSS). They pursue the goal of creating an optimal system through combination and operation of several different ESUs that meet the application-specific requirements best. Most HBSSs that are described in the literature are composed of high energy and high power ESUs to achieve a system with superior characteristics regarding energy, power, system efficiency, fast charging, long lifetime, low cost, low weight, and low volume [491]. In addition to the system-related advantages, the combination of different cell types or cell generations result in further benefits for the manufacturer such as flexible dimensioning and adaption of battery storages, utilization of scale effects (realization of several systems with a fixed number of different cells), or reduction of storage costs (combination of different cell types/generations or aging states).

\subsubsection{Topologies}

An independent control of the energy and power flow of each battery cell of a certain type is essential for the optimal operation and safety of the HBSS. Traditionally, this requires additional power electronics such as one or more unidirectional or bidirectional DC/DC converters. However, with the occurrence of reconfigurable topologies at the cell, module, and system levels, no additional hardware components are required to realize an HBSS. Despite the high potential of HBSSs, there are currently only a few publications that deal with this topic in detail.

First, thoughts on the hybridization of battery storage systems are already found in publications on RBSs and MLIs [442,460,463,492-497]. The most comprehensive and systematic collection of macrotopologies for HBSSs has been published by Zimmermann et al. [498]. As one of the first, the authors combine the two research fields of RBSs and HBSSs and propose a new topology class called discrete hybrid energy storage topologies. The basis for this topology class is formed by ESUs which consist of single, multiple serial, or parallel connected cells. Within a ESU, only one cell type is used such as high power or high energy. The hybridization is realized by using different cell types for ESUs. Besides the energy storage element itself, each ESU also contains some switching elements to activate, bypass, or disable individual units, and therefore allows the reconfiguration of the HBSS as well as an active load management. Based on Zimmermann et al. [497,498], four macrotopologies of multiple ESUs are frequently used for HBSSs: parallel, serial, serial-parallel, and parallel-serial (see Figure 7a-d). The serial macrotopology is used by Horsche et al. [442] to implement a serial HBSS with ESUs on cell level and switches in half bridge configuration.

A different concept is proposed by Tu et al. [492] for the combination of multiple Energy Storage Systems (ESSs) on a system level called series-parallel reconfigurable HBSS. This macrotopology allows for arranging two storage modules in series, parallel, or serial-parallel configuration. However, due to the reconfiguration at system level, this topology results in some restrictions in the operation and dimensioning of an HBSS to avoid high compensation currents.

Guo et al. [494] propose a macrotopology for an HBSS based on the common MMC topology. Therefore, the MMC topology with half bridge microtopology and high power storage units is extended with a separate high energy storage as DC-Link. Thereby, the high energy pack contains no additional switching elements.

Another HBSS topology, also based on the MMC structure, is presented by George et al. [496]. Their concept features a microtopology of the individual ESUs, where each ESU consists of a high energy and high power cells as well as eight switching elements. Four of the switching elements are used to select one or both ESUs (high energy or/and high power) and the remaining four switches form a full bridge to change the polarity of the corresponding ESU. A disadvantage of this concept is the high number of switching elements in the current-carrying path inducing additional losses. 
Ciccarelli et al. [493] provide an MMC approach extending high power storage units with high energy units via an additional DC-link. Furthermore, each ESU integrates a half bridge in conjunction with a DC/DC converter. As a result, a large number of switches and passive components are required in this configuration. Preliminary considerations on HBSSs are found in the publications of Helling et al. [460,463] which introduce the microtopology of BM3 and M2B, as already presented in Section 4.2.1.

In addition to the topologies listed above with reference to HBSSs, many other topologies form the scientific literature on RBSs and especially MLIs are interesting to be used for an HBSS. Particularly promising are topologies that enable the independent control of the energy and power flow within the system and also can generate an AC output voltage. This opens up the possibility to combine the advantages of HBSSs and MLIs, whereby the system efficiency can be increased even further and additional power electronic components can be reduced.

\subsubsection{Operation}

The combination of two or more different cells in one battery system results in a high degree of flexibility in terms of system design and operating strategy. However, the optimal control of the power or energy flow within the HBSS is challenging. A variety of so-called energy management strategies are presented in the scientific literature. Generally, most strategies aim to absorb short-term power peaks with the corresponding high power storages and thus keep the output power of the high energy storage as constant as possible. At the same time, the energy losses are intended to be minimal and simultaneously to extent cyclic lifetime of the high energy storage. As far as the authors are aware, no operating strategy has yet been published that explicitly deals with HBSSs in the context of the novel RBS Type 3 topologies. However, the basic concept of most energy management strategies is applicable to RBSs with some minor changes with regard to power control and current distribution within the HBSS topologies. A compilation of various energy management strategies for an HBSS is given by Krishna et al. [499]. Figure 9 visualizes their categorization. The different algorithms for energy management strategies are classified into four categories: rule-based, filtration-based, optimization-based, and learning-based. This classification is not uniform in the scientific literature and other authors pursue different approaches to grouping and classifying the algorithms.

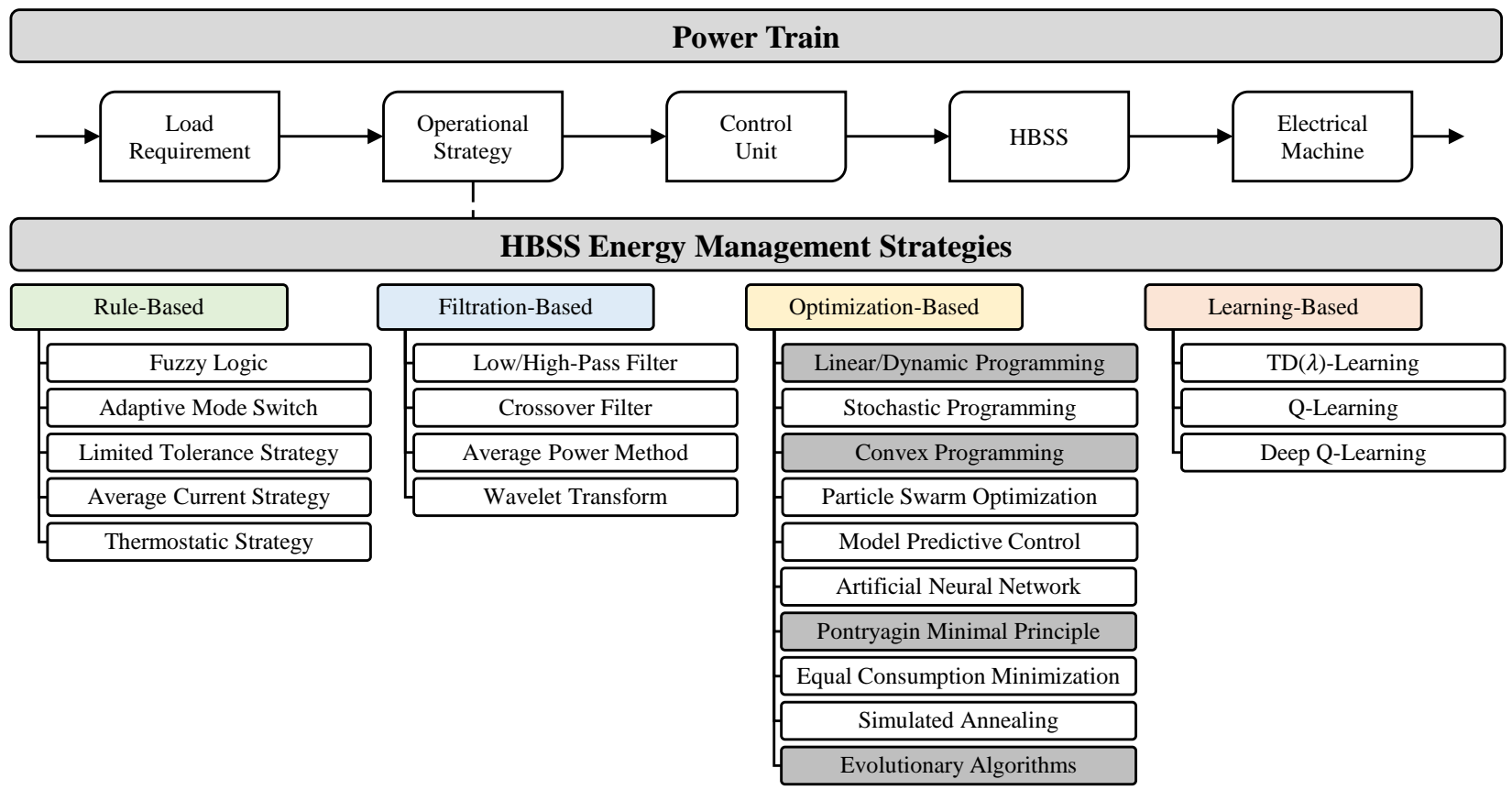

Figure 9. Categorization of different energy management strategies for an HBSS based on [499]. Online strategies are marked with a white background, offline strategies with a gray background. 
The most common are rule-based controllers due to their simplicity in implementation and validation, as well as their good real-time performance. The underlying set of rules are often predefined based on the application and the characteristics of the storage unit. Therefore, the established rules depend either on the expert knowledge of the developer or result from optimization algorithms used to derive the rules. Depending on the underlying algorithms, these can be further subdivided into deterministic and fuzzy logic rule-based strategies [499]. Simple deterministic approaches based on heuristics or empiric experience can be found in [500-502]. The underlying rules are often fixed and presented in a flow chart. Accordingly, the operation strategy can be regarded as a finite state machine. Their performance is heavily dependent on knowledge of the system and its working condition. The same disadvantage also applies to algorithms based on fuzzy logic. One possible way to improve the performance is to use adaptive control strategies that change the underlying set of rules depending on the respective operating point. A relatively simple adaptive strategy, proposed by Jiang et al. [500], includes the setpoint of the output current from the high energy storage depending on the SOC of the high power storage. Zhou et al. [503] introduce another adaptive fuzzy logic based strategy, which changes the output membership function of the fuzzy logic algorithm and the corresponding update frequency according to the past driving cycle pattern. Another very promising approach is to derive the underlying set of rules from the results of a global optimization-based energy management strategy. Promising methods among others used in the literature are dynamic programming [504,505], neural networks [506], and PSO [507,508]. Hu et al. [505] also take traffic information into account.

An alternative variant of energy management strategies are filtration-based also called filter-based or frequency-based approaches, which decompose the power demand of the load into different frequency components to match them to the dynamic response of the involved ESUs. High power cells offer a much higher short-term power capability compared to high energy cells. Therefore, the aim of filtration-based approaches is to stress high energy cells only with the low frequency part of the power demand, while the high power cells take care of the high frequency components. The cutoff frequency and other parameters in the filter design must be set accordingly. Filtration-based strategies have the major disadvantage to introduce a large phase shift [501,509]. A relatively simple filtration-based called average power method is provided by Wang et al. [510]. In order to reduce the operation current/power frequency on the high energy storage, the high power storage acts as a real-time adaptive power filter. For this purpose, the method uses information from both the real-time power and history power demands. Another simple approach to separate high and low frequency components is to apply a crossover filter [511]. The wavelet transformation is also a widely spread mathematical method to analyze and separate different frequency of the power demand in energy management strategies for HBSSs. Using the wavelet transformation, an original signal can be decomposed into components at different positions and scales. Furthermore, the technique can be used in the time as well as frequency-domain [504,512,513]. For an HBSS consisting of three different energy storages, Zhang et al. [512] uses a three-level Haar wavelet to realize an energy management strategy and to allocate power components with different frequency contents. To take the influence of the driving cycle into account, $\mathrm{Hu}$ et al. propose in [513] an adaptive wavelet transform-fuzzy logic control energy management strategy based on driving pattern recognition. Cluster analysis is utilized to classify driving cycles into different patterns according to the features extracted from the historical driving data sampling window, and pattern recognition is used to identify real-time driving patterns. A similar two-level structure-based real-time energy management strategy is proposed by Zhang et al. [504]. It is composed of a learning ANN for driving cycle identification and a multilevel Haar wavelet transform for power demand allocation. Whereby the first level algorithm identifies the type of driving cycles based on a series of statistical characteristic vehicle parameters, the wavelet transform algorithm subsequently uses the information to enhance the power allocation. 
All the aforementioned energy management strategies (rule-based and filtrationbased) are only sufficient to provide a nearly optimal solution under all circumstances. On the contrary, optimization-based approaches are able to deliver the optimal solution for a given power demand profile. However, this often comes at the expense of high computational effort and lack of real-time applicability. The corresponding optimization algorithms can be classified into (a) global optimization and (b) real-time optimization [499]. The global optimization includes algorithms such as linear programming, dynamic programming, convex optimization, and GAs, which can only be applied to real-time problems with adequate modifications or extensions (e.g., an adequate forecasting method). Therefore, global optimization algorithms often serve as a benchmark for the performance of other algorithms or as a starting point to design new strategies [504-508,514,515]. Optimization algorithms that are promising for real-time application include methods such as stochastic dynamic programming, model predictive control, neural networks, simulated annealing, and Pontryagin's minimum principle. However, these algorithms have the disadvantage that they, in turn, can often only achieve nearly-optimal solutions. In order to handle the stochastic uncertainties in operation such as driver-dependent variation of power demand, Wegmann et al. [515] present a real-time stochastic dynamic programming approach based on a stochastic model of the vehicle velocity and power demand. In comparison with the dynamic programming solution, the stochastic dynamic programming strategy shows a comparable performance with the advantage of real-time applicability. In [514], Romaus et al. applied stochastic dynamic programming as well. Since the calculations take up a significant amount of memory, the resolution of the state variables is limited. Therefore, the algorithm results in a look-up table that also can be implemented e.g., on a microcontroller. Further approaches based on model predictive control that make use of a short-term prediction horizon are found in [502,516]. A combination of a rule-based and an optimization-based approach is also feasible. Trovao et al. [517] present an integrated rule-based meta-heuristic approach, where the strategy consists of a long-term rule-based strategy to set the long-term management. It restricts the search space for the optimization-based strategy that optimizes the real-time power distribution without prior knowledge of power demand. The authors use a meta-heuristic simulated annealing approach in [517], while, in [518], a PSO is used within the same framework. In both approaches, the rule-based strategy helps the optimization method to find a good solution quickly, by narrowing the scope of the search to the regions of interest. Choi et al. present in [519] a similar structured real-time control framework based on convex programming.

Another promising category are learning-based energy management strategies, which, however, are up to now rarely considered in the scientific literature. Yue et al. [520] propose a model-free reinforcement learning-based approach. Compared to relatively simple rule-based strategies, the proposed learning-based method delivers the better results. Another approach based on a reinforcement algorithm called Q-learning is introduced by Jiayi et al. [521]. Another rule-based strategy is presented in [522], where the control rules are abstracted from the optimization results with dynamic programming and the learningbased method shows its effectiveness. A more advanced approach present Li et al. [523], which combines reinforcement learning with deep learning. Thereby, the energy management strategy not only aims to minimize the energy loss (as in [520,521]), but also to increase the electrical and thermal safety level of the HBSS.

Due to the great dependency between the design and operation of hybrid storage systems, there are also a large number of publications that have linked these two tasks and solve them together within a multi-objective optimization framework. The common goal of those investigations is to find the global optimum for an HBSS in terms of dimensioning and operation strategy. In contrast, the sole consideration of the operating strategy for a fixed design often only provides a suboptimal solution for the entire HBSS. Yu et al. [524] propose a multi-objective bi-level optimal design and control framework, in order to find the optimal parameters for a fuzzy-logic-based real-time energy management algorithm. This framework is able to obtain the Pareto optimal solutions of the objective function. 
It achieves both the optimal sizing parameters and the static parameters of the energy management system simultaneously for each Pareto optimal solution. The formulated objective function focuses on the reduction of the total mass of the HBSS consisting of a high energy and high power storage and the extension of the cyclic lifetime of the high energy storage. In the subsequent publication [525], Yu et al. enhance the algorithm for the energy management strategy and introduce a parametric vectorized fuzzy inference system, where a large number of fuzzy logic controllers run in parallel and enable fast training and optimization. A different method is presented by Hung et al. [526] with the so-called integrated optimization approach, which iteratively optimizes the system dimensioning and an "if-then-else" rule-based energy management strategy. The approach is implemented within a nested for-loop-structure by solving the different objective functions with a global searching methodology.

The operation strategy has a high degree of flexibility in order to achieve an optimal energy and power distribution within the system. However, this also means a high level of complexity in order to achieve flexibility for all operation conditions and reconcile the interests of various other battery monitoring functions. With this objective and the requirement for real-time capability, optimization-based methods such as stochastic dynamic programming, model predictive control as well as deterministic approaches with rules derived from optimization results are most promising for IBSs. The performance and computation effort of learning-based methods according to the current state of research is difficult to assess due to the limited literature and therefore requires closer investigations and comparisons.

\section{Implementation of Reconfigurable Battery Systems}

The idea of being able to flexibly combine individual battery cells or other ESUs into a favored system configuration shows its attractiveness in many of the applications described in the previous section. However, regardless of the type, an RBS imposes hardware and requirements to be realized in practice. Especially, the selected switching technology influences the feasibility and efficiency of the applied operation strategy. Furthermore, depending on the number of involved ESUs, suitable communication capabilities and an adequate BMS architecture can become a serious challenge to realize the desired functionality. Within this section, questions of practical implementation of RBSs are discussed. Notably, we put emphasize on side effects e.g., thermal influences or aging of switched Li-ion cells that arise in an RBS but is seldom discussed in literature.

\subsection{Switches for Reconfiguration Functionality}

In order to achieve any RBS type, switches are imperative. The correct choice of the switches depends on various factors, such as switching speed and conduction losses. Therefore, it is important to address the general requirements related to the switches. Once this is done, a suitable device among the existing technologies can be chosen.

Generally the switching topology dictates if switches with unidirectional voltage blocking capability [527], bidirectional voltage blocking capability [379], or even a combination of those two [427] is needed. According to Rößler [528], the most important task of the switch is the safe disconnect. Thereby, the operating voltage, load current, pulse current capability, and the option to conduct current in both forward and reverse direction play a crucial role. A low parasitic resistance is desirable as it reduces the conduction losses [529,530]. Manenti et al. [379] states that the switch choice is affected by both the number of components necessary to control the switch and the system's ability to remain safe in every operating point. A general collection of requirements related to switches in reconfigurable battery systems for automotive applications is shown in Table 8. 
Table 8. Requirements related to the switches of RBSs.

Requirement Reasoning

Electric Losses

Leakage Current

Switching Frequency

Blocking Voltage

Current Capability
The electric losses should be low in order to increase the overall range of the BEV and to reduce the required cooling capacity.

Should be low to reduce the stand-by consumption.

For an RBS Type 2, switching frequencies of several kilohertz are appropriate in order to achieve a low harmonic content. In contrast to an RBS Type 2, the switching frequency of a few hertz is sufficient for an RBS Type 1.

The necessary blocking voltage of the switch is determined by the used topology of the RBS [531]. The topology proposed by [532] e.g., requires to block the voltage of the whole battery while the cascaded half-bridge for example only needs to block the voltage of a single cell. Additionally, the topology dictates if the switch has to block voltage in both directions (bidirectional) or in only one direction (unidirectional).

Since batteries need to be charged and discharged, the switch must be able to conduct current in both directions. This requires either a unipolar device or a combination of two bipolar devices or a bipolar device with a Schottky diode in parallel. Additionally, the switch must be able to conduct the peak current of the application.

It should be noted that the choice of the switch practically depends on the maturity of the technology as well as the commercial availability [533]. Therefore, only the most common categories of switches will be discussed.

Electromechanical Relays easily allow for connecting various numbers of ESU in series as they exhibit a galvanic isolation between the control and the power unit. Furthermore, the leakage current during off-state is negligible. The drawbacks of relays are the necessity of an ongoing holding current as well as large physical dimensions and weight compared to semiconductors. Furthermore, the degradation of the contacts due to arcing with each switching operation makes them unsuitable for applications with periodic switching. Since relays are based on mechanical operation, high switching frequencies are not feasible. Nonetheless, the application of relays in the literature can be found [534,535]. It should be noted that even though some research work utilized relays, they suggest to use semiconductor devices instead for the final application. In contrast to electromechanical relays, semiconductor devices exhibit a much lower weight and physical dimensions, while the maximum accessible switching frequency and the reliability are way higher. One drawback of all semiconductors is the need of a drive circuit, which results in additional components. Furthermore, the control signal and power path are not galvanically isolated. This requires an additional digital insulator between the control signal and the switch when multiple stages are connected in series.

Insulated-Gate Bipolar Transistors (IGBTs) achieve switching frequencies of a few tens of kilohertz while showing a very high input resistance. Since the IGBT is a bipolar device, their conduction losses in high-voltage and high-current applications are much lower compared to MOSFETs [536]. One major drawback is that the on-state voltage drop is nearly independent of the actual load current, which results in poor efficiency during light load operation. Furthermore, their switching frequency is limited to a few tens of kilohertz. A third drawback, induced by the bipolarity, is the unidirectional current flow [536]. In order to conduct current in both directions, a freewheeling diode is needed. Applications in the literature that use IGBTs are e.g., [381,537-541].

Thyristors are available in different structures. Only the Gate Turn Off (GTO) thyristor is suitable for DC power circuit applications as the normal thyristor cannot be turned off by removing the control signal [542]. In terms of on-state voltage drop, thyristors behave similar to IGBTs. However, the switching speed of GTOs is very poor and with the invention of IGBTs, the GTO has been replaced in traction applications [542]. Similar 
to the IGBT, it conducts current only in a forward direction and blocks voltage in both directions [536].

Metal-Oxide-Semiconductor Field-Effect Transistors (MOSFETs) achieves high switching speed capability of several hundreds of kilohertz in power applications and have an extremely high input resistance. The on-state voltage drop rises linearly with the load current, thus creating a better efficiency at light load operation than IGBTs. On the contrary, the resistance rises strongly with higher blocking voltage [542] making the technology unsuitable for applications with an operating voltage beyond a few hundred volts. MOSFETs block voltage in only one direction which makes them suitable for DC applications. Furthermore, it can conduct current in both forward and reverse directions [536]. Therefore, MOSFETs are widely used for IBS-functionalities in the literature $[371,375,377,379,380,400,401,441,442,456$, 460,463,529-531,543-551].

Rößler [528] compares thyristors, IGBTs, MOSFETs as well as relays and concludes that thyristors can be excluded for usage in RBSs. According to the author, IGBTs can be used at the cost of poor efficiency and higher cooling effort during light load, whereas MOSFETs is the most promising device especially for low voltage applications where their on-state resistance outperforms any other semiconductor device. Additionally, the possibility of conducting current in both directions without the need of an additional freewheeling diode makes a MOSFET the ideal choice.

Apart from the research work, industrial solutions related to RBSs and MLIs in vehicles are seldom. Drivetek from Switzerland offers an MLI solution based on IGBTs [552]. Their flying capacitor MLI can generate five output levels. Thus, the voltage step size is large enough so that IGBTs are justified.

\subsection{Architecture of the Battery Management System}

The Battery Management System (BMS) is an essential component and is responsible for the safe operation of the battery system. This results in several tasks, from which the most important ones are listed in Table 9.

Table 9. Requirements for the BMS based on [1,553-557].

\begin{tabular}{ll}
\hline Requirement & \multicolumn{1}{c}{ Reasoning } \\
\hline Cell Monitoring & $\begin{array}{l}\text { To keep the battery cells in a save state, parameters like the cell voltages, temperatures, and } \\
\text { currents have to be monitored [558]. }\end{array}$ \\
$\begin{array}{l}\text { Battery Safety and } \\
\text { Protection }\end{array}$ & $\begin{array}{l}\text { No battery cell in the pack may exceed a safety critical limit regarding voltage and tempera- } \\
\text { ture [558]. }\end{array}$ \\
\hline $\begin{array}{l}\text { Estimation of SOC, } \\
\text { SOH, and SOF }\end{array}$ & $\begin{array}{l}\text { SOC, SOH, and SOF are important indicators of battery systems and serve as basis for } \\
\text { functionalities like range prediction, balancing, etc. [554]. }\end{array}$ \\
\hline Ensure the Isolation & The BMS must guarantee the isolation between the HV and the rest of the vehicle. \\
\hline Balancing & $\begin{array}{l}\text { To increase the total usable capacity of the battery pack, charge imbalances between serial } \\
\text { cells must be compensated [554,559]. } \\
\text { The batteries have limitations for the charge current. They have to be considered during the } \\
\text { charging process. Advanced charging strategies (e.g., pulse charging) may be applied. }\end{array}$ \\
\hline Thermal Management & $\begin{array}{l}\text { For safety reason, but also to increase the efficiency, the battery must be operated in an } \\
\text { optimal temperature range. For this purpose, it can be cooled as well as heated. }\end{array}$ \\
Communication with & $\begin{array}{l}\text { The BMS is responsible for sharing important parameters like the SOC of the battery with } \\
\text { the other vehicle's components. Furthermore, power requirements of the drive train are } \\
\text { received. }\end{array}$ \\
the Car & $\begin{array}{l}\text { In emergency case or when the vehicle is parked the BMS opens the main switch. } \\
\text { A significant number of switches are controlled to reconfigure the ESUs. Depending on } \\
\text { the RBS type and its functionality, the switching operation must be performed with high } \\
\text { synchronicity. }\end{array}$ \\
\hline Control RBS switches
\end{tabular}


The architecture of BMSs can be divided generally into centralized and decentralized systems [553]. Additionally, there exist mixture approaches which will be discussed in more detail here. The possible architectures are visualized in Figure 10, and additionally their occurrence in the literature is stated.

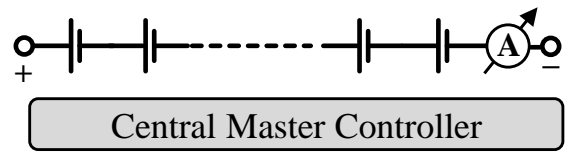

(a) Centralized

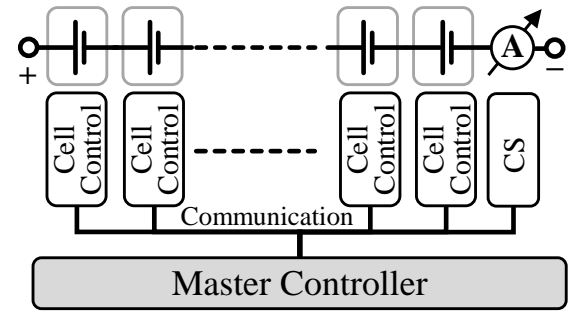

(c) Distributed

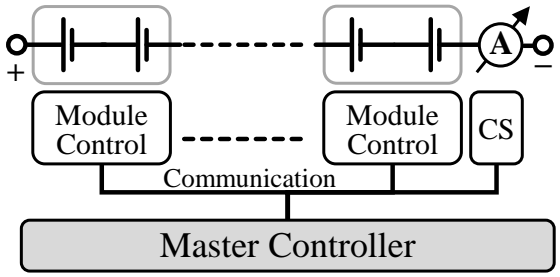

(b) Modularized

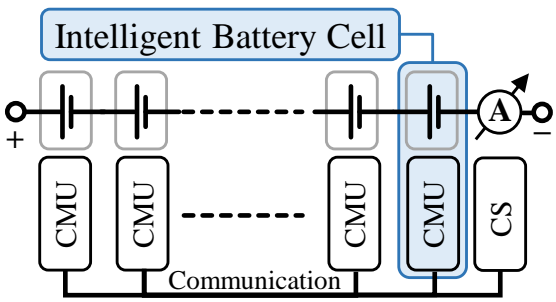

(d) Decentralized

Figure 10. Visualization of different BMS architectures based on [530,560] and their occurrence in literature. (a) centralized [561]; (b) modularized [554,557,562]; (c) distributed [9,401,461,527,555,563-567]; (d) decentralized [553,554,557,560,562,568-570].

Centralized BMSs pool the whole computing power to one controller. With a battery system consisting of a fixed number of Li-ion cells, all monitoring and control tasks are performed on a single computing unit. This is a rather common approach in conventional BMSs. Consequently, temperature and voltage sensors of each cell have to be connected to the central control unit, which causes high wiring effort [553]. The reason for this approach is its low complexity. Since all control algorithms run on the same controller, sensor data and other information are interchangeable between functions. With regard to reconfigurability, synchronicity of switching operation is easier to achieve compared to distributed BMS architectures. However, the central computing unit exhibits significant computation power demands. Consequently, the hardware has to be chosen with respect to the specific battery system to avoid costly undersizing or oversizing, respectively [568]. Therefore, it is difficult to apply the BMS to other battery systems, which results in high development effort due to low flexibility and scalability [530].

Modularized BMSs are built to reduce the wiring effort. In these hierarchical BMS, which commonly are used for large battery systems, computing tasks for sensor data acquisition and cell monitoring are transferred to a module controller, which serves as an intermediate layer between the master controller and the cells [554,557,558,562]. Communication between module and central controller is usually performed via a bus. This modularized approach brings the data acquisition closer to the sensors resulting in more reliable sensing capabilities. Furthermore, the architecture is scalable as more battery modules can be added to the system [553]. However, synchronic switching of reconfigurable battery cells over multiple modules is a challenging task. Furthermore, the advantages of lower communication effort disappear if control algorithm at the master computing unit are driven by cell individual sensor data.

Distributed BMSs pursue the idea of implementing computing power close to the battery cells. They include a controller on each ESU $[401,563,566]$. This can be seen as the basic idea of smart or intelligent battery cells. In [555], each cell is equipped with sensors and a micro controller for data acquisition, $\mathrm{SOC} / \mathrm{SOH}$ determination, and balancing functionality. Furthermore, data storage and communication capabilities are included [565]. Baronti et al. [527] use the storage ability of the cell BMS to store cell individual parameters 
over lifetime. A similar approach is proposed by Lorentz et al. [564]. It enables the usage of the cells for second life application because the aging and corresponding parameter changes can be tracked even if the battery pack is disassembled [530]. Li et al. [561] introduce a distributed battery system with active balancing, where the functionality is implemented on cell level. However, a central BMS controller is still provided for task that are implemented on system level [567]. Communication between cell and central controller is commonly realized by a bus [461]. Distributed BMSs feature a fast response time for cell functionality, which is advantageous for switching operation of RBS, while the data rate of the communication to the master controller is kept low. Furthermore, the measurement accuracy is improved by shorter connection wires and increased interference immunity [553]. Algorithms that are focusing on the individual cell (e.g., SOC and $\mathrm{SOH}$ estimation, safety monitoring, etc.) are implemented on the cell controller, which reduces the computational power requirements of the central BMS Thereby, an energy-efficient BMS can be realized [461]. Though, the information available on system level is limited because raw sensor data are not transferred to the central controller. Consequently, the system algorithms have to deal with reduced input data. Depending on the application, the number of cells in the battery pack can vary, with negligible adjustment of the architecture.

Decentralized BMSs, in contrast, consist of a multitude of identical intelligent battery cells [568]. In this case, no master controller exists as the individual intelligent cells take over all monitoring and control task of the BMS. However, considering that the smart battery cells exchange information with each other, this can lead to an excessive bus load. Consequently, communication has to be limited to a certain degree. With fragmentary information, optimal control is hard to achieve. For decentralized BMSs, the type of algorithms completely differs from central architectures $[530,569]$. System functions like balancing or MLI must be solved in collaboration. Many research questions regarding "concepts of self-organization and collaborative system-level" functionality [568] in battery systems are still unanswered [553]. However, according to Steinhorst et al. [530] and Frost et al. [570], algorithms for active cell balancing are implementable in an efficient manner with such an architecture. Decentralized BMSs surpass central approaches regarding scalability $[560,568]$ because additional cells can be added to the pack with ease, and all cell controller are built in the same way. However, difficulties arise about the connection to other parts of the vehicle as no central communication interface towards the vehicle exists. Decentralized architectures are regarded as robust and reliable because the control and monitoring functions are not relying on specific components which might fail. However, this assumption holds true only in case faulty cells are safely bypassed and cannot damage the rest of the system. Otherwise, the whole battery pack must be disconnected for safety reasons. Therefore, the BBM has to comprise the Li-ion cell itself as well as its corresponding computing power.

To conclude, in today's research regarding IBSs, the trend towards distributed and decentralized BMS architectures is clearly visible. With sensors directly mounted to each $\mathrm{Li}$-ion cell, it is an obvious outgrowth to move computing power for cell monitoring on this level too. Further advantages are the increased scalability and reliability compared to centralized architectures. However, whereas simple operation strategies for e.g., active balancing can be implemented in a decentralized manner, other functionalities still require control of a central master controller. This is true especially for MLI operation, where synchronization of the switching actions is important. With regard to a cost- and energy-efficient solution, the best allocation of computing power is an optimization problem that depends significantly on the specific application. This still remains an open research question.

\subsection{Communication in Intelligent Battery Systems}

Communication of the BMS and among the cells is a major and important element of the application of IBSs with distributed or decentralized architectures. The latter require consideration of communication latencies in order to enable real-time capable realization 
of the features presented in Sections 2 and 3. The quality of some algorithms depend on their cycle time, requiring a high sensor data throughput. MLIs and HBSSs also require low latency for the current control algorithm to achieve a high efficiency of the engine. Unfortunately, most of the literature on the BMS communication do not compare their approaches neither to other solutions nor to the state of the art with regard to metrics like the bitrate, bit error rate, costs, weight, or determinism. Usually, prior literature lack of requirements, communication matrices and measurements under realistic environment and higher number of nodes. Most of the researchers demonstrate the feasibility of their approaches with just a few nodes, whereas BEV may consist of hundreds of nodes with distributed or decentralized architectures. These deficiencies impede a qualitative comparison of the communication systems presented in the literature. Most important requirements regarding the communication are:

- Reliability, due to the high safety requirements.

- Determinism and real-time capability, especially for safety critical data.

- Synchronization, in order to enable synchronous switching in RBSs [571] and to increase the quality of measured data [572]

- Bit rate, which depends on the number of cells, the amount of data to be exchanged per cell and the necessary data update rate of the system.

- Scalability, in order to enable cheap and easy adaption for different vehicles and vehicle variants.

- Security, as the network is a common entry door for hackers.

Reliability and real-time capability are very important for the application in vehicles, since most of the BMS functions are assessed as Automotive Safety Integrity Level (ASIL) [573] $\mathrm{C}$ or D [574] and therefore have to meet strict safety and timing requirements. These points are probably much more important than scalability, as the number of cells in a car is normally fixed. Requirements on the communication further depend on features of the IBS. If cells can be bypassed through switches and, thus, can enter safe states, the criticality of communication losses to individual nodes can be reduced [568] and therefore also the significance of a reliable communication. Furthermore, number and type of sensors strongly influence the necessary bit rate. If the data of current sensors on cell level or the impedance results of EIS must be exchanged, the required bit rate can be significantly higher.

Wired bus topologies are the state-of-the art of BMS communication. However, compared to wireless solutions, complex wiring of more than a hundred nodes leads to difficulty in automated manufacturing [575] (higher production costs), limited flexibility and scalability [576] as well as heavier and larger battery packs [577]. The communication interface must be isolated due to the different voltage levels of serially connected cells [575]. Depending on the maximum voltage difference, this has to be considered for the selection of components and isolation space [9]. The wiring is a potential error source, affecting the reliability in BEVs. Vibrations can lead to physical failure of cables and connectors [575,576]. According to car manufacturers and suppliers, the key failure modes of vehicles are issues with wiring harnesses and connectors [572].

Isolated differential daisy chain connections are usually used with cell monitoring chips such as [578-580]. If the nodes are connected into a ring topology and can forward data in both directions, the system's reliability can be significantly increased through fault tolerance to cable breaks $[578,579]$. Daisy chain is only rarely found in the literature related to BMS communication. In [581], a master-slave network with serially connected Cell Management Units (CMUs) is implemented with isolated Universal Asynchronous Receiver Transmitter (UART). Ring topologies are more likely used for MLI applications [582,583]. EtherCAT is often used due to its exact synchronization feature and to achieve ideal switching precision of $\pm 20 \mathrm{~ns}$. Applying MCUs with integrated EtherCAT in combination with automotive Ethernet [584] is not present in literature, but seems very promising for RBSs, as it is deterministic, requires only two cables, and reaches bit rates up to $100 \mathrm{Mbit} / \mathrm{s}$.

Isolated bus topologies are commonly used for wired intra-battery communication. Stateof-the-art BMSs usually use isolated Controller Area Networks (CANs) $[554,560,568,585,586]$. 
Brandl et al. [554] present galvanic isolation concepts for BMS communication. CAN is very robust, widely used in the automotive industry, cheap, and real-time capable due to its multi-master prioritization and collision resolution scheme [587]. The message filter feature is useful to address individual cells with broadcast communication and to reduce computational load on all other nodes. However, Alonso et al. [588] criticize the poor data rate of ordinary CAN of just $1 \mathrm{Mbit} / \mathrm{s}$. The automotive industry has come up with solutions to that problem. CAN-FD (Flexible Data rate) supports bit rates up to $5 \mathrm{Mbit} / \mathrm{s}$. The upcoming CAN-XL [589] technology from Bosch even features up to $10 \mathrm{Mbit} / \mathrm{s}$. Furthermore, the necessary throughput can be achieved by partitioning Module Management Units (MMUs)/CMUs into separate networks, as long as a distributed master slave topology is applied. Future works on optical CAN and CAN in combination with Ethernet are recommended by Reindl et al. [553]. For the latter case, CAN-XL sounds promising, since it supports up to $2048 \mathrm{~B}$ payload per frame, enabling IP-based protocols [589]. An $\mathrm{I}^{2} \mathrm{C}$ bus is shown in [561] and a UART bus is presented in [590]. Both solutions support master-slave communication. However, these communication systems are much less robust compared to CAN [587]. A novel bidirectional technology based on galvanically isolated, capacitive coupled, differential bus with Frequency Shift Keying (FSK) is presented in $[9,563,564]$. The authors analyzed the communication between up to 100 ESUs. The major advantage of their approach is that isolated contacts and connectors are unnecessary. In order to reduce the number of wires, Zhang et al. [565] propose a novel approach that uses the wiring between the pack managing unit and the CMUs for both power and time division multiplexed data transmission. EMI issues may occur for high numbers of nodes. Unfortunately, literature lacks of measurements on how many nodes can be connected to the same bus, while keeping the communication reliable. This plays an important role for the costs and the network architecture of IBSs.

Power Line Communication (PLC) in EVs utilizes the battery's HV power path as the physical medium to exchange data using coupler circuits. The major benefit compared to standard wired solutions is the reduction of the wiring. Saleem et al. [591] present a BMS with UART over power line with FSK, but the validation comprises only three nodes. Saidani et al. [592] developed a new PLC concept where an AC current source in parallel to the cell is used instead of an inductive or capacitive isolator. Unfortunately, the cell impedance is too small to obtain the necessary amplitude. Using Rogowski coils as coupling elements for PLC, in order to avoid saturation effects of iron-core couplers at high current spikes, is proposed by Opako et al. [593]. The high noise due to inverters and DC/DC converters on the medium is an important issue of PLC [563]. A simulative examination of the physical layer of PLC in EVs is published by Ouannes et al. [594]. The authors modeled a battery with 100 cells and analyzed several modulation schemes without considering noise from the power electronics. Their results show that the attenuation of the master signal strongly depends on the cell's location as the signal propagates through different PLC channels. Furthermore, the busbars used in battery packs do not have a defined impedance characteristic, leading to a lack of scalability and re-usability [594,595]. In [594,595], the authors show that the channel transfer function varies from cell to cell. Unfortunately, it is not investigated how the cell type, cell manufacturer, and cell aging effects influence the transfer functions of the PLC channel. To the best of our knowledge, there are no publications on PLC for RBSs. The additional switches and the varying power path configuration will probably reduce the reliability. MLIs may lead to less interferences, due to the lower switching voltages compared to traditional inverters. Additionally, if the power path is interrupted by switching, the communication will probably break down. Further research is required here.

Wireless Battery Management Systems (WBMSs) are of scientific interest for IBSs as it is naturally isolated and cheap. In addition, it reduces the overall weight of the vehicle due to less wiring [572]. However, safety, availability, reliability, and security have to be fulfilled [596]. Easy access to the network from outside the car is one major disadvantage of WBMS, leading to potential data theft by sniffers, communication losses due to jamming, 
or malicious firmware updates [597]. Unfortunately, most presented WBMSs are not tested under realistic environmental conditions in BEVs. The feasibility of WBMSs in battery packs is thus not proven. Wireless communication issues comprise inference and fading, which can lead to communication losses, so it is seldom applied [565]. For the use in EVs, a deep understanding of the radio channel inside the battery is required [577], which is simulated and investigated for various battery pack parameters by Alonso et al. [598]. The authors analyze different antennas for the application in intra-battery communication in $[577,588]$. Their results show that the channels strongly depend on the pack's geometry and that the distance between the antennas has a high impact on the channels' attenuation. The impact of these parameters increases with higher signal frequencies. Constructing a reliable and stable wireless link inside an EV battery pack is very complex and inflexible which contradicts the advantages of WBMS promised by various publications. However, the usage in other applications like smart grids and ESS might be more promising. The WBMSs presented in the literature use different wireless technologies like ZigBee [599,600], Bluetooth [601], and Wi-Fi [571,602]. An approach where the CMU is integrated inside the cell is proposed by Schneider et al. [566]. Kumtachi et al. [603] suggest a multi-hop forwarding technique based on over-hearing, useful for large scale IBS in smart grids. An Internet of Things (IoT)-based decentralized WBMS architecture is shown by Faika et al. [604]. Unfortunately, the average data aggregation time is over $5 \mathrm{~s}$ for only five nodes. This is obviously too long for safe application in BMSs, where battery data update rates of $10 \mathrm{~Hz}$ are common [572]. Poor real-time capability is also shown by Lafrenz et al. [571] with a WiFi-based WBMS for RBSs. Their measurements show high jitter on the sensor data response time. Lee et al. [575] present a promising master-slave WBMS that can manage up to 780 slave nodes with update rates of $10 \mathrm{~Hz}$. It applies Amplitude Shift Keying (ASK) or FSK modulation for $1 \mathrm{Mbit} / \mathrm{s}$. A contribution by Navitas Solutions Inc. [605] shows that the bitrate already increased to $2 \mathrm{Mbit} / \mathrm{s}$ for up to 640 nodes, communicating temperature and voltage data. Various semiconductor manufacturers work on integrated WBMS solutions. Linear Technology has presented the first WBMS in a BMW i3 concept car in 2016 [606]. General Motors announced to use Analog Devices WBMSs for its automotive Ultium battery platform with the aim to save up to $90 \%$ of the wiring and up to $15 \%$ of the battery pack's volume [607].

It is difficult to determine a specific technology as the best for communication in IBSs. Literature for this topic lacks comparability and realistic conditions, especially with respect to the number of nodes. Wireless communication offers promising advantages but requires a deep understanding of the radio channel. Focusing on MLIs and their requirements on high data throughput, real-time capability, and time synchronous switching, EtherCAT is highly recommendable due to the very precise synchronization, determinism, and high data rate of the technology. Another option is the upcoming CAN-XL, as CAN is very reliable, cheap, and common in the automotive world. CAN-XL further improves CAN networks considering data-rate and payload length. This solution is promising for RBSs with a lower real-time demand compared to MLIs.

\subsection{Further Implications of Reconfiguration}

At this point, further aspects are addressed that are commonly overseen in application of RBSs. Operating physical in contrast to ideal switches implies additional heat in the system and influence on the cell chemistry, but these topics are seldom discussed. Furthermore, the influence of high-frequency switching on lifetime of the cells and efficiency of RBSs is an important topic to guarantee the reliability in an industrial application and justify their application.

\subsubsection{Thermal Influence and Interaction in Reconfigurable Battery Systems}

The main influence for the thermal behavior of battery modules and systems is the local heat generation of cells in combination with the cooling system strategy. In IBS, additional electronic components are utilized at a cell level that generate additional heat 
during use [386,608]. Identifying the heat generating components, mainly sensing (e.g., CSRs) and switching components in the current path generate power losses $[377,609]$. The fact that an intelligent battery system in any case has an increased heat generation is rarely reported and investigated in the literature. It is stated in the context of the number of switches that an increasing number of switches improves the configuration flexibility but increases the power dissipation [373]. In another work, the thermal influence is stated to be negligible [529]. However, in a recent publication, Kleiner et al. [609] investigated the influence of reconfigurable hardware on prismatic cells and found a significant influence on the cell's core temperature. In their follow up work, the authors match the IBS hardware to the automotive application and report a temperature increase of approximately $1 \mathrm{~K}$ due to the power dissipation of the electronics [608]. Thereby, the thermal influence of electronics can be reduced with suitable approaches, but it must not be neglected.

Regarding the thermal modeling of IBS, the consequent approach is to consider the electronics' influence in the model-based estimation of the core temperature. With the electronics influencing the cell's thermal behavior, the influences need to either be represented in the model or to be included in the dataset of solely data-driven approaches.

\subsubsection{Influence of Switching on Cell Lifetime}

During the application in RBS Type 2, such as MLIs, the cells are switched on and off up to the $\mathrm{kHz}$ range. The data base for answering the question on the influence of switching on cell aging is limited as there is a high variety of potential current profiles (waveform, duty cycle, frequency, C-Rate, etc.) and ambient temperature profile. Even for an explicit aging pattern, it is not straight forward to define the appropriate constant current reference test as, e.g., a lower duty cycle increases the test time, reduces the average current, and decreases the temperature rise caused by resistive heat. Therefore, it is not possible to keep all parameters such as average current, maximum current, test time, and cell temperature constant. This makes a comparison of MLIs and reference tests challenging. These fundamental issues of the optimal test design are one explanation for the contradictory conclusions in the various publications on the topic.

The influence on the lifetime of Li-ion batteries for MLI comparable applications, with rectangular waveforms up to the $\mathrm{kHz}$ range, is investigated in only two publications that are known to us $[610,611]$. Uno et al. [611] tested 24 cells with 2 Ah capacity each and a current of $\pm 1 \mathrm{~A}$ for a high-frequency balancer application that is comparable to MLIs. Varying the frequency from $1 \mathrm{~Hz}$ to $100 \mathrm{kHz}$, the authors conclude from the results a higher capacity fade during switching for frequencies below $10 \mathrm{~Hz}$. However, applying switching procedures in the range of $100 \mathrm{~Hz}$ to $100 \mathrm{kHz}$, no significant aging in comparison to calendar life tests is obtained. This was explained by the influence of micro cycles at low frequencies while at higher frequencies only the double layer capacitances of the electrodes are cycled. Chang et al. [610] investigate the MLI application explicitly for a $\mathrm{CHB}$ at $2 \mathrm{kHz}$ and $10 \mathrm{kHz}$. The results for five $3 \mathrm{Ah}$ cells show no dependence on frequency and power factor on capacity fade, but a $2 \%$ lower capacity compared to reference cells with the same average C-rate. Smaller deviations could be explainable by an unintended test pause leading to anode overhang effects that have nearly compensated by the end of the test [612]. The internal resistance changed comparably for all cells. In other literature found for DC/DC converters and fast charging, it is also reported that pulsing in the $\mathrm{kHz}$ range has a negative [613], not significant [614] or positive [615] effect on aging. Korth Pereira Ferraz et al. highlight for $5 \mathrm{kHz}$ a slightly stronger aging for $10 \%$ DOD while no impact is observed for $100 \%$ DOD cycling [616].

As a conclusion, the impact of switching remains unclear, and it is necessary to investigate the influence of switching on the lifetime of the cells for different aging test conditions, high power/high energy cells, and cell chemistries in the upcoming test campaign. 


\subsubsection{Efficient Operation of Reconfigurable Battery Systems}

In addition to the many advantages offered by switches on cell level and reconfigurability at the system level, the influence on the efficiency of the system must not be neglected. This important point is usually not considered or discussed in current publications related to switches and reconfiguration. If at all, the statement is being made that the conduction loss of the used switches needs to be negligibly small to avoid to degrade the performance and the efficiency of the system [375]. Focusing on the influence of switches, Kim et al. [377] mention three approaches to improve the efficiency:

- reduce the internal resistance of a switch by suitable components;

- use multiple switches in parallel for the same functionality;

- $\quad$ reduce the number of single switched ESUs by including multiple cells.

The latter, however, reduces the flexibility of the system. This approach of resistance optimization can be transferred to the other relevant components e.g., by contactless current sensing or high-current capable hardware [608]. Nevertheless, for a meaningful evaluation of the total energy efficiency of the whole EV system, the entire power train must be considered and the lifetime of a vehicle must also be taken into account. The reason is that many advantages and possibilities of intelligent battery systems only become apparent with increasing runtime when cells start to age, faults, and failures occur and weak cells limit the whole system. Especially in this period, when range and performance of conventional battery systems decrease, IBS in their basic functions show their strengths.

Another point of view on efficiency arises when reconfigurable battery systems fundamentally change the architecture of the power train. With MLIs and HBSSs in various forms, conventional power electronics can be replaced. This is of decisive importance for the overall efficiency as switching only takes place at the cell voltage level $(3-5 \mathrm{~V})$ in most RBS cases. In current power electronics architectures, switching is usually performed at the system voltage level of $400-800 \mathrm{~V}$. This opens up the opportunity of using completely different semiconductor components and changing the overall efficiency. Ultimately, however, efficiency can only be assessed for a specific vehicle with real components. A general evaluation of the power loss in RBSs with different topologies and levels of modularization is made by Zimmerman et al. [497].

Besides the electric efficiency of the hardware, the well-designed combination of BMS functionality is relevant due to contrary operation strategies influencing each other. For example, a balancing procedure with respect to SOC homogeneity always changes the cells' temperature and aging coincidently. When switching procedures are necessary for fault detection, EIS excitation, balancing, and MLI procedures simultaneously, a wellcoordinated control and regulation software in the BMS is indispensable.

Consequently, a focus of future research must be on finding operation strategies that combine the discussed functionalities. This is regarded as a multi-objective optimization problem. Thereby, the individual requirements of the advanced sensing functionalities as well as the approaches based on reconfiguration must be intelligently combined with each other to form a holistic IBS.

\section{Summary, Conclusions, and Outline}

Intelligent Battery Systems (IBSs), as a new technological advancement, represent a promising but also a challenging approach to significantly improve the reliability, safety, and efficiency of Battery Electric Vehicles (BEVs). Within this review, the functionalities of IBSs and the prerequisites for their implementation in BEVs have been discussed. Considering the reviewed scientific literature on the functionalities of IBSs, we conclude that, as an emerging technology, IBSs are in a rather early development stage and substantial improvement has to be achieved until a phase of technological maturity is reached.

In this regard, IBSs are a versatile area of research due to their prosperity of available sensor data and manifold control levers. Thereby, intelligent methods can be incorporated in many applications such as continuous adaptive control, aging adaptation, online parameter, and state estimation. In addition to vital operating parameters of individual cells, a 
reliable detection and localization of faults is also achievable. Therefore, the goal of future research related to fault diagnosis must be to bundle the detection of several different errors in a unified fault diagnosis function. Furthermore, in contrast to the well-established State of Charge determination, the existing State of Health monitoring functions must always adapt to the ongoing development of chemical compositions for lithium-ion batteries. This is a general challenge of battery systems. In the context of IBSs, it is an attractive option to use on-board Electrochemical Impedance Spectroscopy for monitoring the aging behavior. However, the hardware implementation for measuring the impedance in a BEV has not been solved yet. In the area of temperature estimation, a major step has already been taken with cell-specific temperature measurement using cell-dedicated hardware. From the economic point of view, algorithm- and model-based approaches for cell temperature estimation seem to be more attractive solutions than cell integrated temperature sensors at present. In general, the realization of IBSs with advanced sensing imposes severe requirements on size, cost, and energy consumption, which is only partly solved by today's commercial sensor technology. Therefore, further research and development regarding simple and yet reliable sensors is encouraged.

With the incorporation of cell individual actuators (switches) into an IBS, a new degree of freedom for optimal and efficient operation is introduced. The resulting Reconfigurable Battery System is a mechatronic system, where intelligent operation strategies can be applied based on sensor data, advanced diagnosis, and overall energy demand. Simple topologies, such as half bridges, already enable basic functionalities such as Bad-Block-Management and balancing. However, when it comes to advanced reconfiguration applications such as Multilevel Inverters (MLIs) or Hybrid Battery Storage Systems, more complex topologies are favorable. The large number of emerging topologies suggests that a detailed analysis of the specific use case is required to be able to find the optimal micro- and macrotopology. For example, if an IBS enables AC output voltage by MLI functionality, external power electronics can be omitted. This in turn requires a topology that enables variable system voltage levels. However, the number of switches in the load path increases costs, losses, and the probability of failure. Moreover, the chosen topology directly affects the thermal interactions as well as the efficiency of the system. Therefore, appropriate semiconductor devices are required to replace the system level power electronics. In addition, the high frequency application of switching for MLIs requires a suited Battery Management System and communication architecture. In addition, finally, all algorithms and functionalities that are based on switching procedures must be coordinated for a functional operation strategy.

Despite the existing challenges, IBSs have the potential to significantly improve the battery systems of Electric Vehicles and provide sophisticated solutions to the many limitations and issues encountered with conventional battery systems. The next important step, besides the continuous development of the individual functionalities of advanced monitoring and Reconfigurable Battery Systems, is to examine the mutual effects of the many functionalities described in the literature to form a holistic concept of Intelligent Battery Systems for future generations of Electric Vehicles.

Supplementary Materials: The following are available online at https:/ /www.mdpi.com/article/10 .3390/en14185989/s1, Table S1: Overview of investigated publications regarding online EIS, part I of II: Excitation and Signal Acquisition; Table S2: Overview of investigated publications regarding online EIS, part II of II: Evaluation and Setup.

Author Contributions: Conceptualization, L.K., J.K. and D.S.; Methodology, L.K., J.K. and D.S.; Writing-original draft preparation, L.K., T.B., S.D., M.E., C.H. (Christian Hanzl), C.H. (Christoph Hartmann), M.H. (Markus Hölzle), J.K., M.L., B.L., M.S., D.S., S.S., J.S. and C.T.; Writing-review and editing, L.K., T.B., S.D., M.E., C.H. (Christian Hanzl), C.H. (Christoph Hartmann), M.H. (Markus Hölzle), J.K., M.L., B.L., M.S., D.S., S.S., J.S., C.T., M.H. (Michael Hinterberger) and C.E.; Visualization, M.H. (Markus Hölzle) and J.K.; Supervision, L.K., M.H. (Michael Hinterberger), and C.E.; Project administration, L.K., M.H. (Michael Hinterberger), and C.E. All authors have read and agreed to the published version of the manuscript. 
Funding: This research was funded by AUDI AG, 85057 Ingolstadt, Germany.

Acknowledgments: The authors express their gratitude to AUDI AG for their support over the past years and the good cooperation.

Conflicts of Interest: The authors declare no conflict of interest.

\section{Abbreviations}

The following abbreviations are used in this manuscript:

AFE Analog Front End

ANN Artificial Neural Network

ASK Amplitude Shift Keying

BBM Bad-Block-Management

BEV Battery Electric Vehicle

BMS Battery Management System

BPF Bandpass Filter

CAN Controller Area Network

CCCT Constant Current Charging Time

CHB Cascaded H-Bridge

CMU Cell Management Unit

CPSD Cross Power Spectral Density

CVCT Constant Voltage Charging Time

CSR Current Sense Resistor

DFT Discrete Fourier Transformation

DKF Dual Kalman Filter

DOD Depth of Discharge

DVA Differential Voltage Analysis

ECM Equivalent Circuit Model

ECIN Enhanced Commutation Integrated Nested

ESC External Short Circuit

EIS Electrochemical Impedance Spectroscopy

EKF Extended Kalman Filter

EMI Electromagnetic Interference

ESU Energy Storage Unit

ESS Energy Storage System

EV Electric Vehicle

FBG Fiber Bragg Grating Sensor

FC Flying Capacitor

FSK Frequency Shift Keying

GA Genetic Algorithm

GPR Gaussian Process Regression

GTO Gate Turn Off

HBSS Hybrid Battery Storage System

HPF High Pass Filter

HPPC Hybrid Pulse Power Characterization

IBS Intelligent Battery System 


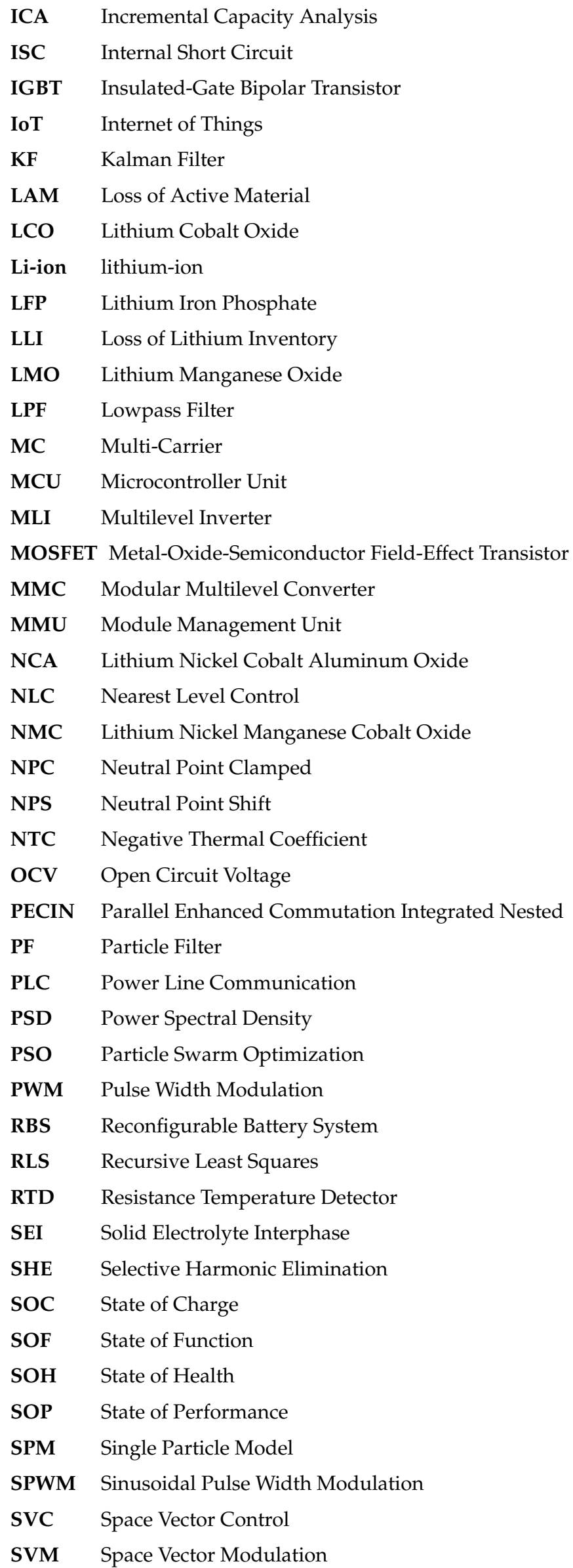




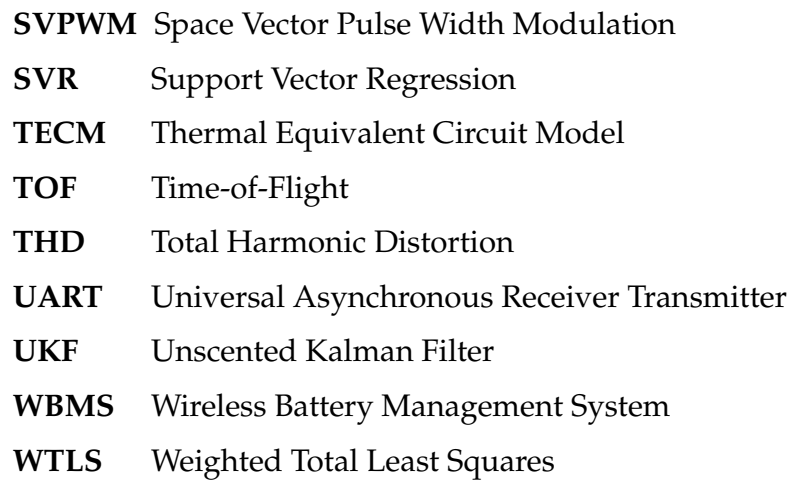

\section{References}

1. Lu, L.; Han, X.; Li, J.; Hua, J.; Ouyang, M. A review on the key issues for lithium-ion battery management in electric vehicles. J. Power Sources 2013, 226, 272-288. [CrossRef]

2. Liu, X.; Ai, W.; Naylor Marlow, M.; Patel, Y.; Wu, B. The effect of cell-to-cell variations and thermal gradients on the performance and degradation of lithium-ion battery packs. Appl. Energy 2019, 248, 489-499. [CrossRef]

3. Campestrini, C.; Keil, P.; Schuster, S.F.; Jossen, A. Ageing of lithium-ion battery modules with dissipative balancing compared with single-cell ageing. J. Energy Storage 2016, 6, 142-152. [CrossRef]

4. Rumpf, K.; Rheinfeld, A.; Schindler, M.; Keil, J.; Schua, T.; Jossen, A. Influence of Cell-to-Cell Variations on the Inhomogeneity of Lithium-Ion Battery Modules. J. Electrochem. Soc. 2018, 165, A2587-A2607. [CrossRef]

5. Hosseinzadeh, E.; Arias, S.; Krishna, M.; Worwood, D.; Barai, A.; Widanalage, D.; Marco, J. Quantifying cell-to-cell variations of a parallel battery module for different pack configurations. Appl. Energy 2021, 282, 115859. [CrossRef]

6. Paul, S.; Diegelmann, C.; Kabza, H.; Tillmetz, W. Analysis of ageing inhomogeneities in lithium-ion battery systems. J. Power Sources 2013, 239, 642-650. [CrossRef]

7. Saidani, F.; Hutter, F.X.; Selinger, W.; Yu, Z.; Burghartz, J.N. A lithium-ion battery demonstrator for HEV applications featuring a smart system at cell level. In Proceedings of the 2017 IEEE International Systems Engineering Symposium (ISSE), Vienna, Austria, 11-13 October 2017; pp. 1-5. [CrossRef]

8. Schneider, D.; Liebhart, B.; Endisch, C. Active state and parameter estimation as part of intelligent battery systems. J. Energy Storage 2021, 39, 102638. [CrossRef]

9. Lorentz, V.; Wenger, M.; Giegerich, M.; Zeltner, S.; März, M.; Frey, L. Smart Battery Cell Monitoring with Contactless Data Transmission. In Advanced Microsystems for Automotive Applications 2012; Meyer, G., Ed.; Springer: Berlin/Heidelberg, Germany, 2012.

10. Kim, T.; Qiao, W.; Qu, L. Series-connected reconfigurable multicell battery: A novel design towards smart batteries. In Proceedings of the 2010 IEEE Energy Conversion Congress and Exposition, Atlanta, GA, USA, 12-16 September 2010; pp. 4257-4263. [CrossRef]

11. Canfield, D. Smart Battery with Integrated Sensing and Electronics. U.S. Patent 20170092994 A1, 30 March 2017.

12. Butzmann, S. Battery Having a Plurality of Battery Cells and Method for Regulating a Battery Voltage of a Battery Using Switchon Probabilities of the Battery Cells. U.S. Patent 9362760 B2, 7 June 2016.

13. Hinterberger, M.; Hellenthal, B. Smart Battery Cell of a Battery for a Motor Vehicle. U.S. Patent 10361466B2, 23 July 2019.

14. Hinterberger, M.; Hellenthal, B. Battery Cell for a Battery of a Motor Vehicle, Battery, and Motor Vehicle. CN 107210418 B, 26 September 2017.

15. Wei, Z.; Zhao, J.; He, H.; Ding, G.; Cui, H.; Liu, L. Future smart battery and management: Advanced sensing from external to embedded multi-dimensional measurement. J. Power Sources 2021, 489, 229462. [CrossRef]

16. Xia, G.; Cao, L.; Bi, G. A review on battery thermal management in electric vehicle application. J. Power Sources 2017, 367, 90-105. [CrossRef]

17. Wu, C.; Zhu, C.; Ge, Y.; Zhao, Y. A Review on Fault Mechanism and Diagnosis Approach for Li-Ion Batteries. J. Nanomater. 2015, 2015, 631263. [CrossRef]

18. Shen, M.; Gao, Q. A review on battery management system from the modeling efforts to its multiapplication and integration. Int J. Energy Res. 2019, 43, 5042-5075. [CrossRef]

19. Kim, I.S. A Technique for Estimating the State of Health of Lithium Batteries Through a Dual-Sliding-Mode Observer. IEEE Trans. Power Electron. 2010, 25, 1013-1022. [CrossRef]

20. Dixon, J.; Pereda, J.; Castillo, C.; Bosch, S. Asymmetrical Multilevel Inverter for Traction Drives Using Only One DC Supply. IEEE Trans. Veh. Technol. 2010, 59, 3736-3743. [CrossRef]

21. Waag, W.; Fleischer, C.; Sauer, D.U. Critical review of the methods for monitoring of lithium-ion batteries in electric and hybrid vehicles. J. Power Sources 2014, 258, 321-339. [CrossRef]

22. Lipu, M.H.; Hannan, M.A.; Hussain, A.; Hoque, M.M.; Ker, P.J.; Saad, M.; Ayob, A. A review of state of health and remaining useful life estimation methods for lithium-ion battery in electric vehicles: Challenges and recommendations. J. Clean. Prod. 2018, 205, 115-133. [CrossRef] 
23. Ripka, P. Electric current sensors: A review. Meas. Sci. Technol. 2010, 21, 112001. [CrossRef]

24. Lyu, D.; Ren, B.; Li, S. Failure modes and mechanisms for rechargeable Lithium-based batteries: A state-of-the-art review. Acta Mech. 2019, 230, 701-727. [CrossRef]

25. Tran, M.K.; Fowler, M. Sensor Fault Detection and Isolation for Degrading Lithium-Ion Batteries in Electric Vehicles Using Parameter Estimation with Recursive Least Squares. Batteries 2020, 6, 1. [CrossRef]

26. Hu, X.; Jiang, Z.; Yan, L.; Yang, G.; Xie, J.; Liu, S.; Zhang, Q.; Xiang, Y.; Min, H.; Peng, X. Real-time visualized battery health monitoring sensor with piezoelectric/pyroelectric poly (vinylidene fluoride-trifluoroethylene) and thin film transistor array by in-situ poling. J. Power Sources 2020, 467, 228367. [CrossRef]

27. Wang, H.; Simunovic, S.; Maleki, H.; Howard, J.N.; Hallmark, J.A. Internal configuration of prismatic lithium-ion cells at the onset of mechanically induced short circuit. J. Power Sources 2016, 306, 424-430. [CrossRef]

28. Diao, W.; Xing, Y.; Saxena, S.; Pecht, M. Evaluation of Present Accelerated Temperature Testing and Modeling of Batteries. Appl. Sci. 2018, 8, 1786. [CrossRef]

29. Xiong, R.; Yu, Q.; Shen, W. Review on sensors fault diagnosis and fault-tolerant techniques for lithium ion batteries in electric vehicles. In Proceedings of the 2018 13th IEEE Conference on Industrial Electronics and Applications (ICIEA), Wuhan, China, 31 May-2 June 2018; pp. 406-410. [CrossRef]

30. Yao, L.; Wang, Z.; Ma, J. Fault detection of the connection of lithium-ion power batteries based on entropy for electric vehicles. J. Power Sources 2015, 293, 548-561. [CrossRef]

31. Offer, G.J.; Yufit, V.; Howey, D.A.; Wu, B.; Brandon, N.P. Module design and fault diagnosis in electric vehicle batteries. J. Power Sources 2012, 206, 383-392. [CrossRef]

32. Schmid, M.; Gebauer, E.; Hanzl, C.; Endisch, C. Active Model-Based Fault Diagnosis in Reconfigurable Battery Systems. IEEE Trans. Power Electron. 2021, 36, 2584-2597. [CrossRef]

33. Lombardi, W.; Zarudniev, M.; Lesecq, S.; Bacquet, S. Sensors fault diagnosis for a BMS. In Proceedings of the 2014 European Control Conference (ECC 2014), Strasbourg, France, 24-27 June 2014; IEEE: Piscataway, NJ, USA, 2014; pp. 952-957. [CrossRef]

34. Schmid, M.; Gebauer, E.; Endisch, C. Structural Analysis in Reconfigurable Battery Systems for Active Fault Diagnosis. IEEE Trans. Power Electron. 2021, 36, 8672-8684. [CrossRef]

35. Xia, B.; Mi, C. A fault-tolerant voltage measurement method for series connected battery packs. J. Power Sources 2016, 308, 83-96. [CrossRef]

36. Xia, B.; Nguyen, T.; Yang, J.; Mi, C. The improved interleaved voltage measurement method for series connected battery packs. J. Power Sources 2016, 334, 12-22. [CrossRef]

37. Kang, Y.; Duan, B.; Zhou, Z.; Shang, Y.; Zhang, C. A multi-fault diagnostic method based on an interleaved voltage measurement topology for series connected battery packs. J. Power Sources 2019, 417, 132-144. [CrossRef]

38. Schmid, M.; Kneidinger, H.G.; Endisch, C. Data-Driven Fault Diagnosis in Battery Systems through Cross-Cell Monitoring. IEEE Sens. J. 2020, 21, 1829-1837. [CrossRef]

39. Schmid, M.; Liebhart, B.; Kleiner, J.; Endisch, C.; Kennel, R. Online Detection of Soft Internal Short Circuits in Lithium-Ion Battery Packs by Data-Driven Cell Voltage Monitoring. In Proceedings of the 2021 IEEE International Conference on Electrical and Electromechanical Energy Conversion (ECCE Asia), Singapore, 24-27 May 2021; pp. 1711-1718. [CrossRef]

40. Dubarry, M.; Truchot, C.; Liaw, B.Y. Cell degradation in commercial $\mathrm{LiFePO}_{4}$ cells with high-power and high-energy designs. J. Power Sources 2014, 258, 408-419. [CrossRef]

41. Dubarry, M.; Liaw, B.Y. Identify capacity fading mechanism in a commercial $\mathrm{LiFePO}_{4}$ cell. J. Power Sources 2009, 194, 541-549. [CrossRef]

42. Bloom, I.; Christophersen, J.; Gering, K. Differential voltage analyses of high-power lithium-ion cells. J. Power Sources 2005, 139, 304-313. [CrossRef]

43. Singh, A.; Izadian, A.; Anwar, S. Fault diagnosis of Li-Ion batteries using multiple-model adaptive estimation. In Proceedings of the 39th annual conference of the IEEE Industrial Electronics Societ, Vienna, Austria, 10-13 November 2013; pp. 3524-3529. [CrossRef]

44. Sidhu, A.; Izadian, A.; Anwar, S. Adaptive Nonlinear Model-Based Fault Diagnosis of Li-Ion Batteries. IEEE Trans. Ind. Electron. 2015, 62, 1002-1011. [CrossRef]

45. Alavi, S.M.M.; Samadi, M.F.; Saif, M. Plating Mechanism Detection in Lithium-ion batteries, by using a particle-filtering based estimation technique. In Proceedings of the 2013 American Control Conference (ACC 2013), Washington, DC, USA, 17-19 June 2013; IEEE: Piscataway, NJ, USA, 2013; pp. 4356-4361. [CrossRef]

46. Tian, J.; Wang, Y.; Chen, Z. Sensor fault diagnosis for lithium-ion battery packs based on thermal and electrical models. Int. J. Electr. Power Energy Syst. 2020, 121, 106087. [CrossRef]

47. Zheng, C.; Chen, Z.; Huang, D. Fault diagnosis of voltage sensor and current sensor for lithium-ion battery pack using hybrid system modeling and unscented particle filter. Energy 2020, 191, 116504. [CrossRef]

48. Lin, T.; Chen, Z.; Zheng, C.; Huang, D.; Zhou, S. Fault diagnosis of lithium-ion battery pack based on hybrid system and dual extended Kalman filter algorithm. IEEE Trans. Transp. Electrif. 2020, 7, 26-36. [CrossRef]

49. Xiong, R.; Yu, Q.; Shen, W.; Lin, C.; Sun, F. A Sensor Fault Diagnosis Method for a Lithium-Ion Battery Pack in Electric Vehicles. IEEE Trans. Power Electron. 2019, 34, 9709-9718. [CrossRef] 
50. Liu, Z.; He, H. Sensor fault detection and isolation for a lithium-ion battery pack in electric vehicles using adaptive extended Kalman filter. Appl. Energy 2017, 185, 2033-2044. [CrossRef]

51. Liu, Z.; Ahmed, Q.; Zhang, J.; Rizzoni, G.; He, H. Structural analysis based sensors fault detection and isolation of cylindrical lithium-ion batteries in automotive applications. Control Eng. Pract. 2016, 52, 46-58. [CrossRef]

52. Liu, Z.; He, H. Model-based Sensor Fault Diagnosis of a Lithium-ion Battery in Electric Vehicles. Energies 2015, 8, 6509-6527. [CrossRef]

53. He, H.; Liu, Z.; Hua, Y. Adaptive Extended Kalman Filter Based Fault Detection and Isolation for a Lithium-Ion Battery Pack. Energy Procedia 2015, 75, 1950-1955. [CrossRef]

54. Gadsden, S.A.; Habibi, S.R. Model-based fault detection of a battery system in a hybrid electric vehicle. In Proceedings of the IEEE Vehicle Power and Propulsion Conference 2011, Chicago, IL, USA, 6-9 September 2011; pp. 1-6. [CrossRef]

55. Dey, S.; Biron, Z.A.; Tatipamula, S.; Das, N.; Mohon, S.; Ayalew, B.; Pisu, P. On-board Thermal Fault Diagnosis of Lithium-ion Batteries For Hybrid Electric Vehicle Application. IFAC-PapersOnLine 2015, 48, 389-394. [CrossRef]

56. Dey, S.; Biron, Z.A.; Tatipamula, S.; Das, N.; Mohon, S.; Ayalew, B.; Pisu, P. Model-based real-time thermal fault diagnosis of Lithium-ion batteries. Control Eng. Pract. 2016, 56, 37-48. [CrossRef]

57. Dey, S.; Perez, H.E.; Moura, S.J. Model-Based Battery Thermal Fault Diagnostics: Algorithms, Analysis, and Experiments. IEEE Trans. Control Syst. Technol. 2019, 27, 576-587. [CrossRef]

58. Xu, J.; Wang, J.; Li, S.; Cao, B. A Method to Simultaneously Detect the Current Sensor Fault and Estimate the State of Energy for Batteries in Electric Vehicles. Sensors 2016, 16, 1328. [CrossRef]

59. Dey, S.; Mohon, S.; Pisu, P.; Ayalew, B. Sensor Fault Detection, Isolation, and Estimation in Lithium-Ion Batteries. IEEE Trans. Control Syst. Technol. 2016, 24, 2141-2149. [CrossRef]

60. Zhang, H.; Pei, L.; Sun, J.; Song, K.; Lu, R.; Zhao, Y.; Zhu, C.; Wang, T. Online Diagnosis for the Capacity Fade Fault of a Parallel-Connected Lithium Ion Battery Group. Energies 2016, 9, 387. [CrossRef]

61. Marcicki, J.; Onori, S.; Rizzoni, G. Nonlinear Fault Detection and Isolation for a Lithium-Ion Battery Management System. In Proceedings of the ASME 2010 Dynamic Systems and Control Conference, Cambridge, MA, USA, 12-15 September 2010; ASME: New York, NY, USA, 2010; pp. 607-614. [CrossRef]

62. Liu, Z.; He, H.; Ahmed, Q.; Rizzoni, G. Structural Analysis Based Fault Detection and Isolation Applied for A Lithium-Ion Battery Pack. IFAC-PapersOnLine 2015, 48, 1465-1470. [CrossRef]

63. Liu, Z.; Ahmed, Q.; Rizzoni, G.; He, H. Fault Detection and Isolation for Lithium-Ion Battery System Using Structural Analysis and Sequential Residual Generation. In Proceedings of the ASME 2014 Dynamic Systems and Control Conference, DSCC 2014, San Antonio, TX, USA, 22-24 October 2014; Volume 2. [CrossRef]

64. Shang, Y.; Lu, G.; Kang, Y.; Zhou, Z.; Duan, B.; Zhang, C. A multi-fault diagnosis method based on modified Sample Entropy for lithium-ion battery strings. J. Power Sources 2020, 446, 227275. [CrossRef]

65. Ma, M.; Wang, Y.; Duan, Q.; Wu, T.; Sun, J.; Wang, Q. Fault detection of the connection of lithium-ion power batteries in series for electric vehicles based on statistical analysis. Energy 2018, 164, 745-756. [CrossRef]

66. Sun, Z.; Liu, P.; Wang, Z. Real-time Fault Diagnosis Method of Battery System Based on Shannon Entropy. Energy Procedia 2017, 105, 2354-2359. [CrossRef]

67. Zheng, Y.; Han, X.; Lu, L.; Li, J.; Ouyang, M. Lithium ion battery pack power fade fault identification based on Shannon entropy in electric vehicles. J. Power Sources 2013, 223, 136-146. [CrossRef]

68. Yao, L.; Fang, Z.; Xiao, Y.; Hou, J.; Fu, Z. An Intelligent Fault Diagnosis Method for Lithium Battery Systems Based on Grid Search Support Vector Machine. Energy 2021, 214, 118866. [CrossRef]

69. Lai, X.; Yi, W.; Kong, X.; Han, X.; Zhou, L.; Sun, T.; Zheng, Y. Online detection of early stage internal short circuits in series-connected lithium-ion battery packs based on state-of-charge correlation. J. Energy Storage 2020, 30, 101514. [CrossRef]

70. Gao, W.; Zheng, Y.; Ouyang, M.; Li, J.; Lai, X.; Hu, X. Micro-Short-Circuit Diagnosis for Series-Connected Lithium-Ion Battery Packs Using Mean-Difference Model. IEEE Trans. Ind. Electron. 2019, 66, 2132-2142. [CrossRef]

71. Feng, X.; Pan, Y.; He, X.; Wang, L.; Ouyang, M. Detecting the internal short circuit in large-format lithium-ion battery using model-based fault-diagnosis algorithm. J. Energy Storage 2018, 18, 26-39. [CrossRef]

72. Chen, W.; Chen, W.T.; Saif, M.; Li, M.F.; Wu, H. Simultaneous Fault Isolation and Estimation of Lithium-Ion Batteries via Synthesized Design of Luenberger and Learning Observers. IEEE Trans. Control Syst. Technol. 2014, 22, 290-298. [CrossRef]

73. Xu, J.; Wang, H.; Shi, H.; Mei, X. Multi-scale short circuit resistance estimation method for series connected battery strings. Energy 2020, 202, 117647. [CrossRef]

74. Meng, J.; Boukhnifer, M.; Delpha, C.; Diallo, D. Incipient short-circuit fault diagnosis of lithium-ion batteries. J. Energy Storage 2020, 31, 101658. [CrossRef]

75. Seo, M.; Park, M.; Song, Y.; Kim, S.W. Online Detection of Soft Internal Short Circuit in Lithium-Ion Batteries at Various Standard Charging Ranges. IEEE Access 2020, 8, 70947-70959. [CrossRef]

76. Seo, M.; Goh, T.; Park, M.; Koo, G.; Kim, S. Detection of Internal Short Circuit in Lithium Ion Battery Using Model-Based Switching Model Method. Energies 2017, 10, 76. [CrossRef]

77. Feng, X.; Weng, C.; Ouyang, M.; Sun, J. Online internal short circuit detection for a large format lithium ion battery. Appl. Energy 2016, 161, 168-180. [CrossRef] 
78. Ouyang, M.; Zhang, M.; Feng, X.; Lu, L.; Li, J.; He, X.; Zheng, Y. Internal short circuit detection for battery pack using equivalent parameter and consistency method. J. Power Sources 2015, 294, 272-283. [CrossRef]

79. Kong, X.; Zheng, Y.; Ouyang, M.; Lu, L.; Li, J.; Zhang, Z. Fault diagnosis and quantitative analysis of micro-short circuits for lithium-ion batteries in battery packs. J. Power Sources 2018, 395, 358-368. [CrossRef]

80. Kim, J.; Cho, B.H. An innovative approach for characteristic analysis and state-of-health diagnosis for a Li-ion cell based on the discrete wavelet transform. J. Power Sources 2014, 260, 115-130. [CrossRef]

81. Xia, B.; Shang, Y.; Nguyen, T.; Mi, C. A correlation based fault detection method for short circuits in battery packs. J. Power Sources 2017, 337, 1-10. [CrossRef]

82. Li, X.; Wang, Z. A novel fault diagnosis method for lithium-Ion battery packs of electric vehicles. Measurement 2018, $116,402-411$. [CrossRef]

83. Liu, P.; Sun, Z.; Wang, Z.; Zhang, J. Entropy-Based Voltage Fault Diagnosis of Battery Systems for Electric Vehicles. Energies 2018, 11, 136. [CrossRef]

84. Wang, Z.; Hong, J.; Liu, P.; Zhang, L. Voltage fault diagnosis and prognosis of battery systems based on entropy and Z -score for electric vehicles. Appl. Energy 2017, 196, 289-302. [CrossRef]

85. Zheng, Y.; Lu, Y.; Gao, W.; Han, X.; Feng, X.; Ouyang, M. Micro-Short-Circuit Cell Fault Identification Method for Lithium-ion Battery Packs Based on the Mutual Information. IEEE Trans. Ind. Electron. 2020, 68, 4373-4381. [CrossRef]

86. Hong, J.; Wang, Z.; Liu, P. Big-Data-Based Thermal Runaway Prognosis of Battery Systems for Electric Vehicles. Energies 2017, 10, 919. [CrossRef]

87. Xue, Q.; Li, G.; Zhang, Y.; Shen, S.; Chen, Z.; Liu, Y. Fault diagnosis and abnormality detection of lithium-ion battery packs based on statistical distribution. J. Power Sources 2021, 482, 228964. [CrossRef]

88. Diao, W.; Naqvi, I.H.; Pecht, M. Early detection of anomalous degradation behavior in lithium-ion batteries. J. Energy Storage 2020, 32, 101710. [CrossRef]

89. Haider, S.N.; Zhao, Q.; Li, X. Data driven battery anomaly detection based on shape based clustering for the data centers class. J. Energy Storage 2020, 29, 101479. [CrossRef]

90. Da, L.; Zhang, Z.; Liu, P.; Wang, Z.; Zhang, L. Battery Fault Diagnosis for Electric Vehicles Based on Voltage Abnormality by Combining the Long Short-Term Memory Neural Network and the Equivalent Circuit Model. IEEE Trans. Power Electron. 2021, 36, 1303-1315. [CrossRef]

91. Ojo, O.; Lang, H.; Kim, Y.; Hu, X.; Mu, B.; Lin, X. A Neural Network-Based Method for Thermal Fault Detection in Lithium-Ion Batteries. IEEE Trans. Ind. Electron. 2020, 68, 4068-4078. [CrossRef]

92. Xiong, J.; Banvait, H.; Li, L.; Chen, Y.; Xie, J.; Liu, Y.; Wu, M.; Chen, J. Failure detection for over-discharged Li-ion batteries. In Proceedings of the 2012 IEEE International Electric Vehicle Conference, Greenville, SC, USA, 4-8 March 2012; pp. 1-5. [CrossRef]

93. Gao, W.; Li, X.; Ma, M.; Fu, Y.; Jiang, J.; Mi, C. Case Study of an Electric Vehicle Battery Thermal Runaway and Online Internal Short Circuit Detection. IEEE Trans. Power Electron. 2020, 36, 2452-2455. [CrossRef]

94. Xia, B.; Chen, Z.; Mi, C.; Robert, B. External short circuit fault diagnosis for lithium-ion batteries. In Proceedings of the IEEE Transportation Electrification Conference and Expo (ITEC), Dearborn, MI, USA, 15-18 June 2014; IEEE: Piscataway, NJ, USA, 2014; pp. 1-7. [CrossRef]

95. Muddappa, V.K.S.; Anwar, S. Electrochemical Model Based Fault Diagnosis of Li-Ion Battery Using Fuzzy Logic. In Proceedings of the ASME International Mechanical Engineering Congress and Exposition, Montreal, QC, Canada, 14-20 November 2014; ASME: New York, NY, USA, 2015. [CrossRef]

96. Kaypmaz, T.C.; Tuncay, R.N. An advanced cell model for diagnosing faults in operation of Li-ion Polymer batteries. In Proceedings of the IEEE Vehicle Power and Propulsion Conference (VPPC), Chicago, IL, USA, 6-9 September 2011; IEEE: Piscataway, NJ, USA, 2011; pp. 1-5. [CrossRef]

97. Yang, R.; Xiong, R.; He, H.; Chen, Z. A fractional-order model-based battery external short circuit fault diagnosis approach for all-climate electric vehicles application. J. Clean. Prod. 2018, 187, 950-959. [CrossRef]

98. Zhao, Y.; Liu, P.; Wang, Z.; Zhang, L.; Hong, J. Fault and defect diagnosis of battery for electric vehicles based on big data analysis methods. Appl. Energy 2017, 207, 354-362. [CrossRef]

99. Schmid, M.; Vögele, U.; Endisch, C. A novel matrix-vector-based framework for modeling and simulation of electric vehicle battery packs. J. Energy Storage 2020, 32, 101736. [CrossRef]

100. Xiong, R.; Sun, F.; Chen, Z.; He, H. A data-driven multi-scale extended Kalman filtering based parameter and state estimation approach of lithium-ion polymer battery in electric vehicles. Appl. Energy 2014, 113, 463-476. [CrossRef]

101. Wang, Q.; Kang, J.; Tan, Z.; Luo, M. An online method to simultaneously identify the parameters and estimate states for lithium ion batteries. Electrochim. Acta 2018, 289, 376-388. [CrossRef]

102. Wei, Z.; Zhao, J.; Zou, C.; Lim, T.M.; Tseng, K.J. Comparative study of methods for integrated model identification and state of charge estimation of lithium-ion battery. J. Power Sources 2018, 402, 189-197. [CrossRef]

103. Wang, Y.; Zhao, L.; Cheng, J.; Zhou, J.; Wang, S. A State of Charge Estimation Method of Lithium-Ion Battery Based on Fused Open Circuit Voltage Curve. Appl. Sci. 2020, 10, 1264. [CrossRef]

104. Meng, J.; Boukhnifer, M.; Diallo, D.; Wang, T. A New Cascaded Framework for Lithium-Ion Battery State and Parameter Estimation. Appl. Sci. 2020, 10, 1009. [CrossRef] 
105. Dai, H.; Wei, X.; Sun, Z. Recursive Parameter Identification of Lithium-Ion Battery for EVs Based on Equivalent Circuit Model. J. Comput. Theor. Nanosci. 2013, 10, 2813-2818. [CrossRef]

106. Xia, J.; Dai, H.; Sun, Z.; Venturi, M. Parameter Identification of Battery Pack Considering Cell Inconsistency. In Proceedings of the SAE World Congress Experience 2017, Detroit, MI, USA, 4-6 April 2017. [CrossRef]

107. Wen, F.; Duan, B.; Zhang, C.; Zhu, R.; Shang, Y.; Zhang, J. High-Accuracy Parameter Identification Method for Equivalent-Circuit Models of Lithium-Ion Batteries Based on the Stochastic Theory Response Reconstruction. Electronics 2019, 8, 834. [CrossRef]

108. Wei, Z.; Zou, C.; Leng, F.; Soong, B.H.; Tseng, K.J. Online Model Identification and State-of-Charge Estimate for Lithium-Ion Battery With a Recursive Total Least Squares-Based Observer. IEEE Trans. Ind. Electron. 2018, 65, 1336-1346. [CrossRef]

109. Chiang, Y.H.; Sean, W.Y.; Ke, J.C. Online estimation of internal resistance and open-circuit voltage of lithium-ion batteries in electric vehicles. J. Power Sources 2011, 196, 3921-3932. [CrossRef]

110. Ali, M.U.; Zafar, A.; Nengroo, S.H.; Hussain, S.; Kim, H.J. Effect of Sensors Sensitivity on Lithium-Ion Battery Modeled Parameters and State of Charge: A Comparative Study: State of charge (SOC); sensitivity analysis; current sensor precision; voltage sensor precision. Electronics 2019, 8, 709. [CrossRef]

111. Pang, H.; Zhang, F. Experimental Data-Driven Parameter Identification and State of Charge Estimation for a Li-Ion Battery Equivalent Circuit Model. Energies 2018, 11, 1033. [CrossRef]

112. Liebhart, B.; Komsiyska, L.; Endisch, C. Passive impedance spectroscopy for monitoring lithium-ion battery cells during vehicle operation. J. Power Sources 2020, 449, 227297. [CrossRef]

113. Troxler, Y.; Wu, B.; Marinescu, M.; Yufit, V.; Patel, Y.; Marquis, A.J.; Brandon, N.P.; Offer, G.J. The effect of thermal gradients on the performance of lithium-ion batteries. J. Power Sources 2014, 247, 1018-1025. [CrossRef]

114. Arora, S.; Shen, W.; Kapoor, A. Neural network based computational model for estimation of heat generation in $\mathrm{LiFePO}_{4}$ pouch cells of different nominal capacities. Comput. Chem. Eng. 2017, 101, 81-94. [CrossRef]

115. Cui, X.; Zeng, J.; Zhang, H.; Yang, J.; Qiao, J.; Li, J.; Li, W. Optimization of the lumped parameter thermal model for hard-cased li-ion batteries. J. Energy Storage 2020, 32, 101758. [CrossRef]

116. Lundgren, H.; Svens, P.; Ekström, H.; Tengstedt, C.; Lindström, J.; Behm, M.; Lindbergh, G. Thermal Management of Large-Format Prismatic Lithium-Ion Battery in PHEV Application. J. Electrochem. Soc. 2016, 163, A309-A317. [CrossRef]

117. Kleiner, J.; Komsiyska, L.; Elger, G.; Endisch, C. Thermal Modelling of a Prismatic Lithium-Ion Cell in a Battery Electric Vehicle Environment: Influences of the Experimental Validation Setup. Energies 2020, 13, 62. [CrossRef]

118. Baumann, M.; Rohr, S.; Lienkamp, M. Cloud-connected battery management for decision-making on second-life of electric vehicle batteries. In Proceedings of the 2018 Thirteenth International Conference, Monte Carlo, Monaco, 10-12 April 2018; pp. 1-6. [CrossRef]

119. Jiang, J.; Ruan, H.; Sun, B.; Zhang, W.; Gao, W.; Le Wang, Y.; Zhang, L. A reduced low-temperature electro-thermal coupled model for lithium-ion batteries. Appl. Energy 2016, 177, 804-816. [CrossRef]

120. Richardson, R.R.; Ireland, P.T.; Howey, D.A. Battery internal temperature estimation by combined impedance and surface temperature measurement. J. Power Sources 2014, 265, 254-261. [CrossRef]

121. Richardson, R.R.; Zhao, S.; Howey, D.A. On-board monitoring of 2D spatially-resolved temperatures in cylindrical lithium-ion batteries: Part I. Low-order thermal modelling. J. Power Sources 2016, 326, 377-388. [CrossRef]

122. Forgez, C.; Vinh Do, D.; Friedrich, G.; Morcrette, M.; Delacourt, C. Thermal modeling of a cylindrical $\mathrm{LiFePO}_{4} / \mathrm{graphite}$ lithium-ion battery. J. Power Sources 2010, 195, 2961-2968. [CrossRef]

123. Damay, N.; Forgez, C.; Bichat, M.P.; Friedrich, G. Thermal modeling of large prismatic $\mathrm{LiFePO}_{4} /$ graphite battery. Coupled thermal and heat generation models for characterization and simulation. J. Power Sources 2015, 283, 37-45. [CrossRef]

124. Zhao, Y.; Patel, Y.; Zhang, T.; Offer, G.J. Modeling the Effects of Thermal Gradients Induced by Tab and Surface Cooling on Lithium Ion Cell Performance. J. Electrochem. Soc. 2018, 165, A3169-A3178. [CrossRef]

125. Li, Y.; Chen, J.; Lan, F. Enhanced online model identification and state of charge estimation for lithium-ion battery under noise corrupted measurements by bias compensation recursive least squares. J. Power Sources 2020, 456, 227984. [CrossRef]

126. Liu, Z.; Du, J.; Stimming, U.; Wang, Y. Adaptive observer design for the cell temperature estimation in battery packs in electric vehicles. In Proceedings of the 2014 IEEE 9th Conference on Industrial Electronics and Applications (ICIEA 2014), Hangzhou, China, 9-11 June 2014; IEEE: Piscataway, NJ, USA, 2014; pp. 348-353. [CrossRef]

127. Lin, X.; Fu, H.; Perez, H.E.; Siege, J.B.; Stefanopoulou, A.G.; Ding, Y.; Castanier, M.P. Parameterization and Observability Analysis of Scalable Battery Clusters for Onboard Thermal Management. Oil Gas Sci. Technol.-Rev. D'Ifp Energies Nouv. 2013, 68, 165-178. [CrossRef]

128. Kleiner, J.; Komsiyska, L.; Elger, G.; Endisch, C. Real-time core temperature prediction of prismatic automotive li-ion battery cells based on artificial neural networks. J. Energy Storage 2021, 39, 102588. [CrossRef]

129. Kleiner, J.; Komsiyska, L.; Elger, G.; Endisch, C. Advanced thermal state monitoring and temperature prediction in electric vehicles by physical-based and data-driven modeling of intelligent battery cells. Batteries 2021, 7, 31. [CrossRef]

130. Panchal, S.; Dincer, I.; Agelin-Chaab, M.; Fraser, R.; Fowler, M. Thermal modeling and validation of temperature distributions in a prismatic lithium-ion battery at different discharge rates and varying boundary conditions. Appl. Therm. Eng. 2016, 96, 190-199. [CrossRef]

131. Panchal, S.; Mathew, M.; Dincer, I.; Agelin-Chaab, M.; Fraser, R.; Fowler, M. Thermal and electrical performance assessments of lithium-ion battery modules for an electric vehicle under actual drive cycles. Electr. Power Syst. Res. 2018, 163, 18-27. [CrossRef] 
132. Hussein, A.A.; Chehade, A.A. Robust Artificial Neural Network-Based Models for Accurate Surface Temperature Estimation of Batteries. IEEE Trans. Ind. Appl. 2020, 56, 5269-5278. [CrossRef]

133. Srinivasan, R.; Carkhuff, B.G.; Butler, M.H.; Baisden, A.C. Instantaneous measurement of the internal temperature in lithium-ion rechargeable cells. Electrochim. Acta 2011, 56, 6198-6204. [CrossRef]

134. Raijmakers, L.; Danilov, D.L.; Eichel, R.A.; Notten, P. A review on various temperature-indication methods for Li-ion batteries. Appl. Energy 2019, 240, 918-945. [CrossRef]

135. Richardson, R.R.; Howey, D.A. Sensorless Battery Internal Temperature Estimation Using a Kalman Filter With Impedance Measurement. IEEE Trans. Sustain. Energy 2015, 6, 1190-1199. [CrossRef]

136. Srinivasan, R. Monitoring dynamic thermal behavior of the carbon anode in a lithium-ion cell using a four-probe technique. $J$. Power Sources 2012, 198, 351-358. [CrossRef]

137. Srinivasan, R.; Demirev, P.A.; Carkhuff, B.G. Rapid monitoring of impedance phase shifts in lithium-ion batteries for hazard prevention. J. Power Sources 2018, 405, 30-36. [CrossRef]

138. Beelen, H.; Raijmakers, L.; Donkers, M.; Notten, P.; Bergveld, H.J. An Improved Impedance-Based Temperature Estimation Method for Li-ion Batteries. IFAC-PapersOnLine 2015, 48, 383-388. [CrossRef]

139. Beelen, H.; Raijmakers, L.; Donkers, M.; Notten, P.; Bergveld, H.J. A comparison and accuracy analysis of impedance-based temperature estimation methods for Li-ion batteries. Appl. Energy 2016, 175, 128-140. [CrossRef]

140. Zhu, J.G.; Sun, Z.C.; Wei, X.Z.; Dai, H.F. A new lithium-ion battery internal temperature online estimate method based on electrochemical impedance spectroscopy measurement. J. Power Sources 2015, 274, 990-1004. [CrossRef]

141. Zhu, J.; Sun, Z.; Wei, X.; Dai, H. Battery Internal Temperature Estimation for $\mathrm{LiFePO}_{4}$ Battery Based on Impedance Phase Shift under Operating Conditions. Energies 2017, 10, 60. [CrossRef]

142. Liebhart, B.; Diehl, S.; Endisch, C. Sensitivity Analysis of Battery Cell Aging Estimators based on Impedance Spectroscopy regarding Temperature Compensation. In Proceedings of the 2020 IEEE Conference on Control Technology and Applications (CCTA), Montreal, QC, Canada, 24-26 August 2020; pp. 801-806. [CrossRef]

143. Beelen, H.; Mundaragi Shivakumar, K.; Raijmakers, L.; Donkers, M.; Bergveld, H.J. Towards impedance-based temperature estimation for Li-ion battery packs. Int. J. Energy Res. 2020, 44, 2889-2908. [CrossRef]

144. Raijmakers, L.; Danilov, D.L.; van Lammeren, J.; Lammers, M.; Notten, P. Sensorless battery temperature measurements based on electrochemical impedance spectroscopy. J. Power Sources 2014, 247, 539-544. [CrossRef]

145. Raijmakers, L. Sensorless Temperature Measurements for Advanced Battery Management Systems. Ph.D. Thesis, Delft University of Technology, Delft, The Netherlands, 2018.

146. Ranieri, M.; Alberto, D.; Piret, H.; Cattin, V. Electronic module for the thermal monitoring of a Li-ion battery cell through the electrochemical impedance estimation. In Proceedings of the THERMINIC 2016 -22nd International Workshop Thermal Investigations of ICs and Systems, Budapest, Hungary, 21-23 September 2016; IEEE: Piscataway, NJ, USA, 2016 ; pp. $294-297$. [CrossRef]

147. Raijmakers, L.H.J.; Danilov, D.L.; van Lammeren, J.P.M.; Lammers, T.J.G.; Bergveld, H.J.; Notten, P.H.L. Non-Zero Intercept Frequency: An Accurate Method to Determine the Integral Temperature of Li-Ion Batteries. IEEE Trans. Ind. Electron. 2016, 63, 3168-3178. [CrossRef]

148. Socher, S.; Jehle, C.; Potthoff, U. Improving the Functional Safety of Automotive Batteries Using in-situ Impedance Spectroscopy. Transp. Res. Procedia 2016, 14, 3661-3666. [CrossRef]

149. Carkhuff, B.G.; Demirev, P.A.; Srinivasan, R. Impedance-Based Battery Management System for Safety Monitoring of Lithium-Ion Batteries. IEEE Trans. Ind. Electron. 2018, 65, 6497-6504. [CrossRef]

150. Haussmann, P.; Melbert, J. Internal Cell Temperature Measurement and Thermal Modeling of Lithium Ion Cells for Automotive Applications by Means of Electrochemical Impedance Spectroscopy. SAE Int. J. Altern. Powertrains 2017, 6, 261-270. [CrossRef]

151. Morello, R.; Di Rienzo, R.; Roncella, R.; Saletti, R.; Schwarz, R.; Lorentz, V.; Hoedemaekers, E.; Rosca, B.; Baronti, F. Advances in Li-Ion Battery Management for Electric Vehicles. In Proceedings of the IECON 2018 - 44th Annual Conference of the IEEE Industrial Electronics Society, Washington, DC, USA, 21-23 October 2018; pp. 4949-4955. [CrossRef]

152. Richardson, R.R.; Zhao, S.; Howey, D.A. On-board monitoring of 2D spatially-resolved temperatures in cylindrical lithium-ion batteries: Part II. State estimation via impedance-based temperature sensing. J. Power Sources 2016, 327, 726-735. [CrossRef]

153. Schmidt, J.P.; Arnold, S.; Loges, A.; Werner, D.; Wetzel, T.; Ivers-Tiffée, E. Measurement of the internal cell temperature via impedance: Evaluation and application of a new method. J. Power Sources 2013, 243, 110-117. [CrossRef]

154. Schwarz, R.; Semmler, K.; Wenger, M.; Lorentz, V.R.H.; Marz, M. Sensorless battery cell temperature estimation circuit for enhanced safety in battery systems. In Proceedings of the IECON 2015-Yokohama, Yokohama, Japan, 9-12 November 2015; Ohishi, K., Hashimoto, H., Eds.; IEEE: Piscataway, NJ, USA, 2015; pp. 001536-001541. [CrossRef]

155. Spinner, N.S.; Love, C.T.; Rose-Pehrsson, S.L.; Tuttle, S.G. Expanding the Operational Limits of the Single-Point Impedance Diagnostic for Internal Temperature Monitoring of Lithium-ion Batteries. Electrochim. Acta 2015, 174, 488-493. [CrossRef]

156. Wang, L.; Lu, D.; Song, M.; Zhao, X.; Li, G. Instantaneous estimation of internal temperature in lithium-ion battery by impedance measurement. Int. J. Energy Res. 2020, 44, 3082-3097. [CrossRef]

157. Wang, X.; Wei, X.; Dai, H.; Wu, Q. State Estimation of Lithium Ion Battery Based on Electrochemical Impedance Spectroscopy with On-Board Impedance Measurement System. In Proceedings of the 2015 IEEE Vehicle Power and Propulsion Conference (VPPC), Montreal, QC, Canada, 19-22 October 2015; IEEE: Piscataway, NJ, USA, 2015; pp. 1-5. [CrossRef] 
158. Wang, X.; Wei, X.; Chen, Q.; Zhu, J.; Dai, H. Lithium-ion battery temperature online estimation based on fast impedance calculation. J. Energy Storage 2019, 26, 100952. [CrossRef]

159. Rivera-Barrera, J.; Muñoz-Galeano, N.; Sarmiento-Maldonado, H. SoC Estimation for Lithium-ion Batteries: Review and Future Challenges. Electronics 2017, 6, 102. [CrossRef]

160. Shrivastava, P.; Soon, T.K.; Idris, M.Y.I.B.; Mekhilef, S. Overview of model-based online state-of-charge estimation using Kalman filter family for lithium-ion batteries. Renew. Sustain. Energy Rev. 2019, 113, 109233. [CrossRef]

161. Plett, G.L. Efficient Battery Pack State Estimation using Bar-Delta Filtering. In Proceedings of the International Battery, Hybrid and Fuel Cell Electric Vehicle Symposium, Stavanger, Norway, 13-16 May 2009; pp. 1-8.

162. Roscher, M.A.; Bohlen, O.S.; Sauer, D.U. Reliable State Estimation of Multicell Lithium-Ion Battery Systems. IEEE Trans. Energy Convers. 2011, 26, 737-743. [CrossRef]

163. Dai, H.; Wei, X.; Sun, Z.; Wang, J.; Gu, W. Online cell SOC estimation of Li-ion battery packs using a dual time-scale Kalman filtering for EV applications. Appl. Energy 2012, 95, 227-237. [CrossRef]

164. Zheng, Y.; Ouyang, M.; Lu, L.; Li, J.; Han, X.; Xu, L.; Ma, H.; Dollmeyer, T.A.; Freyermuth, V. Cell state-of-charge inconsistency estimation for $\mathrm{LiFePO}_{4}$ battery pack in hybrid electric vehicles using mean-difference model. Appl. Energy 2013, 111, 571-580. [CrossRef]

165. Sun, F.; Xiong, R.; He, H. A systematic state-of-charge estimation framework for multi-cell battery pack in electric vehicles using bias correction technique. Appl. Energy 2016, 162, 1399-1409. [CrossRef]

166. Yang, C.; Wang, X.; Fang, Q.; Dai, H.; Cao, Y.; Wei, X. An online SOC and capacity estimation method for aged lithium-ion battery pack considering cell inconsistency. J. Energy Storage 2020, 29, 101250. [CrossRef]

167. Zhong, L.; Zhang, C.; He, Y.; Chen, Z. A method for the estimation of the battery pack state of charge based on in-pack cells uniformity analysis. Appl. Energy 2014, 113, 558-564. [CrossRef]

168. Chun, C.Y.; Baek, J.; Seo, G.S.; Cho, B.H.; Kim, J.; Chang, I.K.; Lee, S. Current sensor-less state-of-charge estimation algorithm for lithium-ion batteries utilizing filtered terminal voltage. J. Power Sources 2015, 273, 255-263. [CrossRef]

169. Fleischer, C.; Waag, W.; Heyn, H.M.; Sauer, D.U. On-line adaptive battery impedance parameter and state estimation considering physical principles in reduced order equivalent circuit battery models. J. Power Sources 2014, 260, 276-291. [CrossRef]

170. Moura, S.J.; Chaturvedi, N.A.; Krstic, M. PDE estimation techniques for advanced battery management systems—Part I: SOC estimation. In Proceedings of the 2012 American Control Conference (ACC), Montreal, QC, Canada, 27-29 June 2012; pp. 559-565. [CrossRef]

171. Xu, J.; Mi, C.C.; Cao, B.; Deng, J.; Chen, Z.; Li, S. The State of Charge Estimation of Lithium-Ion Batteries Based on a ProportionalIntegral Observer. IEEE Trans. Veh. Technol. 2014, 63, 1614-1621. [CrossRef]

172. Kim, I.S. The novel state of charge estimation method for lithium battery using sliding mode observer. J. Power Sources 2006, 163, 584-590. [CrossRef]

173. Kim, T.; Qiao, W.; Qu, L. Online SOC and SOH estimation for multicell lithium-ion batteries based on an adaptive hybrid battery model and sliding-mode observer. In Proceedings of the IEEE Energy Conversion Congress and Exposition (ECCE), Denver, CO, USA, 15-19 September 2013; IEEE: Piscataway, NJ, USA, 2013; pp. 292-298. [CrossRef]

174. Hu, C.; Youn, B.D.; Chung, J. A multiscale framework with extended Kalman filter for lithium-ion battery SOC and capacity estimation. Appl. Energy 2012, 92, 694-704. [CrossRef]

175. Kim, J.; Cho, B.H. State-of-Charge Estimation and State-of-Health Prediction of a Li-Ion Degraded Battery Based on an EKF Combined With a Per-Unit System. IEEE Trans. Veh. Technol. 2011, 60, 4249-4260. [CrossRef]

176. Mastali, M.; Vazquez-Arenas, J.; Fraser, R.; Fowler, M.; Afshar, S.; Stevens, M. Battery state of the charge estimation using Kalman filtering. J. Power Sources 2013, 239, 294-307. [CrossRef]

177. Plett, G.L. Extended Kalman filtering for battery management systems of LiPB-based HEV battery packs: Part 3 . State and parameter estimation. J. Power Sources 2004, 134, 277-292. [CrossRef]

178. Santhanagopalan, S.; White, R.E. Online estimation of the state of charge of a lithium ion cell. J. Power Sources 2006, 161, 1346-1355. [CrossRef]

179. Lai, X.; Qin, C.; Gao, W.; Zheng, Y.; Yi, W. A State of Charge Estimator Based Extended Kalman Filter Using an ElectrochemistryBased Equivalent Circuit Model for Lithium-Ion Batteries. Appl. Sci. 2018, 8, 1592. [CrossRef]

180. Li, W.; Fan, Y.; Ringbeck, F.; Jöst, D.; Han, X.; Ouyang, M.; Sauer, D.U. Electrochemical model-based state estimation for lithium-ion batteries with adaptive unscented Kalman filter. J. Power Sources 2020, 476, 228534. [CrossRef]

181. Zheng, Y.; Ouyang, M.; Han, X.; Lu, L.; Li, J. Investigating the error sources of the online state of charge estimation methods for lithium-ion batteries in electric vehicles. J. Power Sources 2018, 377, 161-188. [CrossRef]

182. Lin, C.; Yu, Q.; Xiong, R.; Le Wang, Y. A study on the impact of open circuit voltage tests on state of charge estimation for lithium-ion batteries. Appl. Energy 2017, 205, 892-902. [CrossRef]

183. Pop, V.; Bergveld, H.J.; Op het Veld, J.H.G.; Regtien, P.P.L.; Danilov, D.; Notten, P.H.L. Modeling Battery Behavior for Accurate State-of-Charge Indication. J. Electrochem. Soc. 2006, 153, A2013. [CrossRef]

184. Walder, G.; Campestrini, C.; Lienkamp, M.; Jossen, A. Adaptive State and Parameter Estimation of Lithium-Ion Batteries Based on a Dual Linear Kalman Filter. In Proceedings of the Second International Conference on Technological Advances in Electrical, Electronics and Computer Engineering, Kuala Lumpur, Malaysia, 18-20 March 2014; pp. 16-24. 
185. Zou, Y.; Hu, X.; Ma, H.; Li, S.E. Combined State of Charge and State of Health estimation over lithium-ion battery cell cycle lifespan for electric vehicles. J. Power Sources 2015, 273, 793-803. [CrossRef]

186. Zou, C.; Manzie, C.; Nešić, D.; Kallapur, A.G. Multi-time-scale observer design for state-of-charge and state-of-health of a lithium-ion battery. J. Power Sources 2016, 335, 121-130. [CrossRef]

187. Rubagotti, M.; Onori, S.; Rizzoni, G. Automotive Battery Prognostics Using Dual Extended Kalman Filter. In Proceedings of the ASME 2009 Dynamic Systems and Control Conference, Hollywood, CA, USA, 12-14 October 2009; ASME: New York, NY, USA, 2009; Volume 2, pp. 257-263. [CrossRef]

188. Sun, F.; Xiong, R.; He, H. Estimation of state-of-charge and state-of-power capability of lithium-ion battery considering varying health conditions. J. Power Sources 2014, 259, 166-176. [CrossRef]

189. Kim, J.; Cho, B.H. Pattern Recognition for Temperature-Dependent State-of-Charge/Capacity Estimation of a Li-ion Cell. IEEE Trans. Energy Convers. 2013, 28, 1-11. [CrossRef]

190. Maletić, F.; Hrgetić, M.; Deur, J. Dual Nonlinear Kalman Filter-Based SoC and Remaining Capacity Estimation for an Electric Scooter Li-NMC Battery Pack. Energies 2020, 13, 540. [CrossRef]

191. Dragicevic, T.; Sucic, S.; Guerrero, J.M. Battery state-of-charge and parameter estimation algorithm based on Kalman filter. In Proceedings of the Eurocon 2013, Zagreb, Croatia, 1-4 July 2013; pp. 1519-1525. [CrossRef]

192. Andre, D.; Appel, C.; Soczka-Guth, T.; Sauer, D.U. Advanced mathematical methods of SOC and SOH estimation for lithium-ion batteries. J. Power Sources 2013, 224, 20-27. [CrossRef]

193. Guo, F.; Hu, G.; Hong, R. A parameter adaptive method with dead zone for state of charge and parameter estimation of lithium-ion batteries. J. Power Sources 2018, 402, 174-182. [CrossRef]

194. Plett, G.L. Sigma-point Kalman filtering for battery management systems of LiPB-based HEV battery packs: Part 2: Simultaneous state and parameter estimation. J. Power Sources 2006, 161, 1369-1384. [CrossRef]

195. Zhang, F.; Rehman, M.M.U.; Wang, H.; Levron, Y.; Plett, G.; Zane, R.; Maksimovic, D. State-of-charge estimation based on microcontroller-implemented sigma-point Kalman filter in a modular cell balancing system for Lithium-Ion battery packs. In Proceedings of the 2015 IEEE 16th Workshop on Control and Modeling for Power Electronics (COMPEL), Vancouver, BC, Canada, 12-15 July 2015; IEEE: Piscataway, NJ, USA, 2015; pp. 1-7. [CrossRef]

196. Xiong, R.; Sun, F.; Gong, X.; He, H. Adaptive state of charge estimator for lithium-ion cells series battery pack in electric vehicles. J. Power Sources 2013, 242, 699-713. [CrossRef]

197. Sun, F.; Hu, X.; Zou, Y.; Li, S. Adaptive unscented Kalman filtering for state of charge estimation of a lithium-ion battery for electric vehicles. Energy 2011, 36, 3531-3540. [CrossRef]

198. Shen, P.; Ouyang, M.; Lu, L.; Li, J.; Feng, X. The Co-estimation of State of Charge, State of Health, and State of Function for Lithium-Ion Batteries in Electric Vehicles. IEEE Trans. Veh. Technol. 2018, 67, 92-103. [CrossRef]

199. Xu, W.; Xu, J.; Yan, X. Lithium-ion battery state of charge and parameter joint estimation using cubature Kalman filter and particle filter. J. Power Electron. 2020, 20, 292-307. [CrossRef]

200. Ganeshan, A.; Shanmughasundaram, R. Estimation of SOC and SOH using Mixed Neural Network and Coulomb Counting Algorithm. Int. J. Innov. Technol. Explor. Eng. 2019, 8, 2557-2561. [CrossRef]

201. Rahbari, O.; Omar, N.; van den Bossche, P.; van Mierlo, J. A centralized state of charge estimation technique for electric vehicles equipped with lithium-ion batteries in smart grid environment. In Proceedings of the 2018 IEEE International Conference on Industrial Technology (ICIT), Lyon, France, 20-22 February 2018; pp. 1721-1725. [CrossRef]

202. Yang, F.; Li, W.; Li, C.; Miao, Q. State-of-charge estimation of lithium-ion batteries based on gated recurrent neural network. Energy 2019, 175, 66-75. [CrossRef]

203. Zahid, T.; Xu, K.; Li, W.; Li, C.; Li, H. State of charge estimation for electric vehicle power battery using advanced machine learning algorithm under diversified drive cycles. Energy 2018, 162, 871-882. [CrossRef]

204. Li, R.; Xu, S.; Li, S.; Zhou, Y.; Zhou, K.; Liu, X.; Yao, J. State of Charge Prediction Algorithm of Lithium-Ion Battery Based on PSO-SVR Cross Validation. IEEE Access 2020, 8, 10234-10242. [CrossRef]

205. Shi, Q.S.; Zhang, C.H.; Cui, N.X. Estimation of battery state-of-charge using v-support vector regression algorithm. Int. J. Automot. Technol. 2008, 9, 759-764. [CrossRef]

206. Ozcan, G.; Pajovic, M.; Sahinoglu, Z.; Wang, Y.; Orlik, P.V.; Wada, T. Online battery state-of-charge estimation based on sparse gaussian process regression. In Proceedings of the 2016 IEEE Power and Energy Society General Meeting (PESGM), Boston, MA, USA, 17-21 July 2016; pp. 1-5. [CrossRef]

207. Howey, D.A.; Mitcheson, P.D.; Yufit, V.; Offer, G.J.; Brandon, N.P. Online Measurement of Battery Impedance Using Motor Controller Excitation. IEEE Trans. Veh. Technol. 2014, 63, 2557-2566. [CrossRef]

208. Jansen, P.; Vergossen, D.; Renner, D.; John, W.; Götze, J. Impedance spectra classification for determining the state of charge on a lithium iron phosphate cell using a support vector machine. Adv. Radio Sci. 2015, 13, 127-132. [CrossRef]

209. Mohan, S.; Kim, Y.; Siegel, J.B.; Samad, N.A.; Stefanopoulou, A.G. A Phenomenological Model of Bulk Force in a Li-Ion Battery Pack and Its Application to State of Charge Estimation. J. Electrochem. Soc. 2014, 161, A2222-A2231. [CrossRef]

210. Mohan, S.; Kim, Y.; Stefanopoulou, A.G. On Improving Battery State of Charge Estimation Using Bulk Force Measurements. In Proceedings of the ASME 2015 Dynamic Systems and Control Conference, Columbus, OH, USA, 28-30 October 2015; Volume 1, Paper No. V001T13A010. [CrossRef] 
211. Ganguli, A.; Saha, B.; Raghavan, A.; Kiesel, P.; Arakaki, K.; Schuh, A.; Schwartz, J.; Hegyi, A.; Sommer, L.W.; Lochbaum, A.; et al. Embedded fiber-optic sensing for accurate internal monitoring of cell state in advanced battery management systems part 2 : Internal cell signals and utility for state estimation. J. Power Sources 2017, 341, 474-482. [CrossRef]

212. Ghannoum, A.; Norris, R.C.; Iyer, K.; Zdravkova, L.; Yu, A.; Nieva, P. Optical Characterization of Commercial Lithiated Graphite Battery Electrodes and in Situ Fiber Optic Evanescent Wave Spectroscopy. ACS Appl. Mater. Interfaces 2016, 8, 18763-18769. [CrossRef]

213. Ghannoum, A.; Nieva, P.; Yu, A.; Khajepour, A. Development of Embedded Fiber-Optic Evanescent Wave Sensors for Optical Characterization of Graphite Anodes in Lithium-Ion Batteries. ACS Appl. Mater. Interfaces 2017, 9, 41284-41290. [CrossRef]

214. Modrzynski, C.; Roscher, V.; Rittweger, F.; Ghannoum, A.; Nieva, P.; Riemschneider, K.R. Integrated Optical Fibers for Simultaneous Monitoring of the Anode and the Cathode in Lithium Ion Batteries. In Proceedings of the IEEE SENSORS 2019, Montreal, QC, Canada, 27-30 October 2019; IEEE: Piscataway, NJ, USA, 2019; pp. 1-4. [CrossRef]

215. Birkl, C.R.; Roberts, M.R.; McTurk, E.; Bruce, P.G.; Howey, D.A. Degradation diagnostics for lithium ion cells. J. Power Sources 2017, 341, 373-386. [CrossRef]

216. Lewerenz, M.; Marongiu, A.; Warnecke, A.; Sauer, D.U. Differential voltage analysis as a tool for analyzing inhomogeneous aging: A case study for $\mathrm{LiFePO}_{4} \mid$ Graphite cylindrical cells. J. Power Sources 2017, 368, 57-67. [CrossRef]

217. Haifeng, D.; Xuezhe, W.; Zechang, S. A new SOH prediction concept for the power lithium-ion battery used on HEVs. In Proceedings of the IEEE Vehicle Power and Propulsion Conference, Dearborn, MI, USA, 7-10 September 2009; IEEE: Piscataway, NJ, USA, 2009; pp. 1649-1653. [CrossRef]

218. Berecibar, M.; Gandiaga, I.; Villarreal, I.; Omar, N.; van Mierlo, J.; van den Bossche, P. Critical review of state of health estimation methods of Li-ion batteries for real applications. Renew. Sustain. Energy Rev. 2016, 56, 572-587. [CrossRef]

219. Xiong, R.; Li, L.; Tian, J. Towards a smarter battery management system: A critical review on battery state of health monitoring methods. J. Power Sources 2018, 405, 18-29. [CrossRef]

220. Tian, H.; Qin, P.; Li, K.; Zhao, Z. A review of the state of health for lithium-ion batteries: Research status and suggestions. J. Clean. Prod. 2020, 261, 120813. [CrossRef]

221. Li, Y.; Liu, K.; Foley, A.M.; Zülke, A.; Berecibar, M.; Nanini-Maury, E.; van Mierlo, J.; Hoster, H.E. Data-driven health estimation and lifetime prediction of lithium-ion batteries: A review. Renew. Sustain. Energy Rev. 2019, 113, 109254. [CrossRef]

222. Prasad, G.K.; Rahn, C.D. Model based identification of aging parameters in lithium ion batteries. J. Power Sources 2013, $232,79-85$. [CrossRef]

223. Guo, Z.; Qiu, X.; Hou, G.; Liaw, B.Y.; Zhang, C. State of health estimation for lithium ion batteries based on charging curves. J. Power Sources 2014, 249, 457-462. [CrossRef]

224. Liu, H.; Naqvi, I.H.; Li, F.; Liu, C.; Shafiei, N.; Li, Y.; Pecht, M. An analytical model for the CC-CV charge of Li-ion batteries with application to degradation analysis. J. Energy Storage 2020, 29, 101342. [CrossRef]

225. Eddahech, A.; Briat, O.; Vinassa, J.M. Determination of lithium-ion battery state-of-health based on constant-voltage charge phase. J. Power Sources 2014, 258, 218-227. [CrossRef]

226. Lee, S.; Kim, J.; Lee, J.; Cho, B.H. State-of-charge and capacity estimation of lithium-ion battery using a new open-circuit voltage versus state-of-charge. J. Power Sources 2008, 185, 1367-1373. [CrossRef]

227. Feng, X.; Li, J.; Ouyang, M.; Lu, L.; Li, J.; He, X. Using probability density function to evaluate the state of health of lithium-ion batteries. J. Power Sources 2013, 232, 209-218. [CrossRef]

228. Li, Y.; Abdel-Monem, M.; Gopalakrishnan, R.; Berecibar, M.; Nanini-Maury, E.; Omar, N.; van den Bossche, P.; van Mierlo, J. A quick online state of health estimation method for Li-ion battery with incremental capacity curves processed by Gaussian filter. $J$. Power Sources 2018, 373, 40-53. [CrossRef]

229. Theiler, M.; Endisch, C.; Lewerenz, M. Float Current Analysis for Fast Calendar Aging Assessment of $18650 \mathrm{Li}(\mathrm{NiCoAl}) \mathrm{O} 2 / \mathrm{Graphite}$ Cells. Batteries 2021, 7, 22. [CrossRef]

230. Tröltzsch, U.; Kanoun, O.; Tränkler, H.R. Characterizing aging effects of lithium ion batteries by impedance spectroscopy. Electrochim. Acta 2006, 51, 1664-1672. [CrossRef]

231. Huhman, B.M. A Single-Frequency Impedance Diagnostic for State of Health Determination in Li-ion 4P1S Battery Packs. Ph.D. Thesis, Virginia State University, Falls Church, VA, USA, 2017.

232. Lajara, R.; Perez-Solano, J.J.; Pelegri-Sebastia, J. Predicting the batteries' state of health in wireless sensor networks applications. IEEE Trans. Ind. Electron. 2018, 65, 8936-8945. [CrossRef]

233. Gong, W.; Chen, Y.; Kou, L.; Kang, R.; Yang, Y. Life Prediction of Lithium Ion Batteries for Electric Vehicles Based on Gas Production Behavior Model. In Proceedings of the 2017 International Conference on Sensing, Diagnostics, Prognostics, and Control, Shanghai, China, 16-18 August 2017; Li, C., Ed.; IEEE: Piscataway, NJ, USA, 2017; pp. 275-280. [CrossRef]

234. Li, Y.; Wei, Z.; Xiong, B.; Vilathgamuwa, D.M. Adaptive Ensemble-Based Electrochemical-Thermal-Degradation State Estimation of Lithium-Ion Batteries. IEEE Trans. Ind. Electron. 2021, 1. [CrossRef]

235. Chiang, Y.H.; Sean, W.Y. Dynamical estimation of State-of-Health of batteries by using adaptive observer. In Proceedings of the 2009 2nd International Conference on Power Electronics and Intelligent Transportation System (PEITS), Shenzhen, China, 19-20 December 2009; pp. 110-115. [CrossRef]

236. Plett, G.L. Recursive approximate weighted total least squares estimation of battery cell total capacity. J. Power Sources 2011, 196, 2319-2331. [CrossRef] 
237. Remmlinger, J.; Buchholz, M.; Meiler, M.; Bernreuter, P.; Dietmayer, K. State-of-health monitoring of lithium-ion batteries in electric vehicles by on-board internal resistance estimation. J. Power Sources 2011, 196, 5357-5363. [CrossRef]

238. Rahimian, S.K.; Rayman, S.; White, R.E. State of Charge and Loss of Active Material Estimation of a Lithium Ion Cell under Low Earth Orbit Condition Using Kalman Filtering Approaches. J. Electrochem. Soc. 2012, 159, A860. [CrossRef]

239. Nuhic, A.; Terzimehic, T.; Soczka-Guth, T.; Buchholz, M.; Dietmayer, K. Health diagnosis and remaining useful life prognostics of lithium-ion batteries using data-driven methods. J. Power Sources 2013, 239, 680-688. [CrossRef]

240. Remmlinger, J.; Buchholz, M.; Soczka-Guth, T.; Dietmayer, K. On-board state-of-health monitoring of lithium-ion batteries using linear parameter-varying models. J. Power Sources 2013, 239, 689-695. [CrossRef]

241. Schwunk, S.; Straub, S.; Armbruster, N.; Matting, S.; Vetter, M. Parallel particle filter for state of charge and health estimation with a long term test. In Proceedings of the World Electric Vehicle Symposium and Exposition (EVS 27), Barcelona, Spain, 17-20 November 2013; IEEE: Piscataway, NJ, USA, 2013; pp. 1-10. [CrossRef]

242. Weng, C.; Cui, Y.; Sun, J.; Peng, H. On-board state of health monitoring of lithium-ion batteries using incremental capacity analysis with support vector regression. J. Power Sources 2013, 235, 36-44. [CrossRef]

243. Zheng, Y.; Lu, L.; Han, X.; Li, J.; Ouyang, M. $\mathrm{LiFePO}_{4}$ battery pack capacity estimation for electric vehicles based on charging cell voltage curve transformation. J. Power Sources 2013, 226, 33-41. [CrossRef]

244. Han, X.; Ouyang, M.; Lu, L.; Li, J. A comparative study of commercial lithium ion battery cycle life in electric vehicle: Capacity loss estimation. J. Power Sources 2014, 268, 658-669. [CrossRef]

245. Hu, X.; Li, S.E.; Jia, Z.; Egardt, B. Enhanced sample entropy-based health management of Li-ion battery for electrified vehicles. Energy 2014, 64, 953-960. [CrossRef]

246. Berecibar, M.; Omar, N.; Garmendia, M.; Dubarry, M.; Villarreal, I.; van den Bossche, P.; van Mierlo, J. SOH Estimation and Prediction for NMC Cells Based on Degradation Mechanism Detection. In Proceedings of the 2015 IEEE Vehicle Power and Propulsion Conference (VPPC), Montreal, QC, Canada, 19-22 October 2015; pp. 1-6. [CrossRef]

247. Wu, J.; Wang, Y.; Zhang, X.; Chen, Z. A novel state of health estimation method of Li-ion battery using group method of data handling. J. Power Sources 2016, 327, 457-464. [CrossRef]

248. Dubarry, M.; Berecibar, M.; Devie, A.; Anseán, D.; Omar, N.; Villarreal, I. State of health battery estimator enabling degradation diagnosis: Model and algorithm description. J. Power Sources 2017, 360, 59-69. [CrossRef]

249. Sánchez, L.; Couso, I.; Otero, J.; Echevarría, Y.; Anseán, D.; Sánchez, L.; Couso, I.; Otero, J.; Echevarría, Y.; Anseán, D. A Model-Based Virtual Sensor for Condition Monitoring of Li-Ion Batteries in Cyber-Physical Vehicle Systems. J. Sens. 2017, 2017, 9643279. [CrossRef]

250. Cai, Y.; Yang, L.; Deng, Z.; Zhao, X.; Deng, H. Online identification of lithium-ion battery state-of-health based on fast wavelet transform and cross D-Markov machine. Energy 2018, 147, 621-635. [CrossRef]

251. Chen, Z.; Sun, M.; Shu, X.; Shen, J.; Xiao, R. On-board state of health estimation for lithium-ion batteries based on random forest. In Proceedings of the 2018 IEEE International Conference on Industrial Technology (ICIT), Lyon, France, 20-22 February 2018; pp. 1754-1759. [CrossRef]

252. Li, S.; Pischinger, S.; He, C.; Liang, L.; Stapelbroek, M. A comparative study of model-based capacity estimation algorithms in dual estimation frameworks for lithium-ion batteries under an accelerated aging test. Appl. Energy 2018, 212, 1522-1536. [CrossRef]

253. Santos, S.R.d.; Aranha, J.C.M.S.; Nascimento, T.C.d.; Vieira, D.; Junior, E.M.O.; Cerri, F. Study of machine learning algorithms to state of health estimation of iron phosphate lithium-ion battery used in fully electric vehicles. In Proceedings of the 2018 SAE Brasil Congress \& Exhibition, Sao Paulo, Brazil, 3-5 September 2018. [CrossRef]

254. Smiley, A.; Plett, G.L. An adaptive physics-based reduced-order model of an aged lithium-ion cell, selected using an interacting multiple-model Kalman filter. J. Energy Storage 2018, 19, 120-134. [CrossRef]

255. Tang, X.; Zou, C.; Yao, K.; Chen, G.; Liu, B.; He, Z.; Gao, F. A fast estimation algorithm for lithium-ion battery state of health. J. Power Sources 2018, 396, 453-458. [CrossRef]

256. Wassiliadis, N.; Adermann, J.; Frericks, A.; Pak, M.; Reiter, C.; Lohmann, B.; Lienkamp, M. Revisiting the dual extended Kalman filter for battery state-of-charge and state-of-health estimation: A use-case life cycle analysis. J. Energy Storage 2018, $19,73-87$. [CrossRef]

257. Yu, Q.; Xiong, R.; Yang, R.; Pecht, M.G. Online capacity estimation for lithium-ion batteries through joint estimation method. Appl. Energy 2019, 255, 113817. [CrossRef]

258. Zheng, Y.; Qin, C.; Lai, X.; Han, X.; Xie, Y. A novel capacity estimation method for lithium-ion batteries using fusion estimation of charging curve sections and discrete Arrhenius aging model. Appl. Energy 2019, 251, 113327. [CrossRef]

259. Bi, Y.; Yin, Y.; Choe, S.Y. Online state of health and aging parameter estimation using a physics-based life model with a particle filter. J. Power Sources 2020, 476, 228655. [CrossRef]

260. Jiang, B.; Dai, H.; Wei, X. Incremental capacity analysis based adaptive capacity estimation for lithium-ion battery considering charging condition. Appl. Energy 2020, 269, 115074. [CrossRef]

261. Shu, X.; Li, G.; Zhang, Y.; Shen, J.; Chen, Z.; Liu, Y. Online diagnosis of state of health for lithium-ion batteries based on short-term charging profiles. J. Power Sources 2020, 471, 228478. [CrossRef]

262. Xu, J.; Mei, X.; Wang, X.; Fu, Y.; Zhao, Y.; Wang, J. A Relative State of Health Estimation Method Based on Wavelet Analysis for Lithium-Ion Battery Cells. IEEE Trans. Ind. Electron. 2020, 1. [CrossRef] 
263. Yang, J.; Du, C.; Liu, W.; Wang, T.; Yan, L.; Gao, Y.; Cheng, X.; Zuo, P.; Ma, Y.; Yin, G.; et al. State-of-health estimation for satellite batteries based on the actual operating parameters-Health indicator extraction from the discharge curves and state estimation. $J$. Energy Storage 2020, 31, 101490. [CrossRef]

264. Meissner, E.; Richter, G. Battery Monitoring and Electrical Energy Management. J. Power Sources 2003, 116, 79-98. [CrossRef]

265. Farmann, A.; Sauer, D.U. A comprehensive review of on-board State-of-Available-Power prediction techniques for lithium-ion batteries in electric vehicles. J. Power Sources 2016, 329, 123-137. [CrossRef]

266. Sun, F.; Xiong, R.; He, H.; Li, W.; Aussems, J.E.E. Model-based dynamic multi-parameter method for peak power estimation of lithium-ion batteries. Appl. Energy 2012, 96, 378-386. [CrossRef]

267. Xiong, R.; Sun, F.; He, H.; Nguyen, T.D. A data-driven adaptive state of charge and power capability joint estimator of lithium-ion polymer battery used in electric vehicles. Energy 2013, 63, 295-308. [CrossRef]

268. Bohlen, O.; Buller, S.; de Doncker, R.W.; Gelbke, M.; Naumann, R. Impedance based battery diagnosis for automotive applications. In Proceedings of the 2004 IEEE 35th Annual Power Electronics Specialists Conference (IEEE Cat. No.04CH37551), Aachen, Germany, 20-25 June 2004; IEEE: Piscataway, NJ, USA, 2004; pp. 2792-2797. [CrossRef]

269. Plett, G.L. High-Performance Battery-Pack Power Estimation Using a Dynamic Cell Model. IEEE Trans. Veh. Technol. 2004, 53, 1586-1593. [CrossRef]

270. Küpper, M.; Hülshorst, T.; Seibert, D. Prädiktive Algorithmen für Lithium-Ionen-Traktionsbatterien in Elektro- und Hybridfahrzeugen. ATZelektronik 2011, 6, 70-78. [CrossRef]

271. Anderson, R.D.; Zhao, Y.; Wang, X.; Yang, X.G.; Li, Y. Real time battery power capability estimation. In Proceedings of the 2012 American Control Conference (ACC), Montreal, QC, Canada, 27-29 June 2012; pp. 592-597. [CrossRef]

272. Bhattacharya, S.; Bauer, P. Requirements for charging of an electric vehicle system based on state of power (SoP) and state of energy (SoE). In Proceedings of the 7th International Power Electronics and Motion Control Conference, Harbin, China, 2-5 June 2012; pp. 434-438. [CrossRef]

273. Xiong, R.; He, H.; Sun, F.; Zhao, K. Online Estimation of Peak Power Capability of Li-Ion Batteries in Electric Vehicles by a Hardware-in-Loop Approach. Energies 2012, 5, 1455-1469. [CrossRef]

274. Fleischer, C.; Waag, W.; Bai, Z.; Sauer, D.U. Adaptive On-line State-of-available-power Prediction of Lithium-ion Batteries. J. Power Electron. 2013, 13, 516-527. [CrossRef]

275. Pei, L.; Zhu, C.; Wang, T.; Lu, R.; Chan, C.C. Online peak power prediction based on a parameter and state estimator for lithium-ion batteries in electric vehicles. Energy 2014, 66, 766-778. [CrossRef]

276. Balagopal, B.; Chow, M.Y. The state of the art approaches to estimate the state of health (SOH) and state of function (SOF) of lithium Ion batteries. In Proceedings of the 2015 IEEE 13th International Conference on Industrial Informatics (INDIN), Cambridge, UK, 22-24 July 2015; pp. 1302-1307. [CrossRef]

277. Xavier, M.A.; Trimboli, M.S. Lithium-ion battery cell-level control using constrained model predictive control and equivalent circuit models. J. Power Sources 2015, 285, 374-384. [CrossRef]

278. Burgos-Mellado, C.; Orchard, M.E.; Kazerani, M.; Cárdenas, R.; Sáez, D. Particle-filtering-based estimation of maximum available power state in Lithium-Ion batteries. Appl. Energy 2016, 161, 349-363. [CrossRef]

279. Dong, G.; Wei, J.; Chen, Z. Kalman filter for onboard state of charge estimation and peak power capability analysis of lithium-ion batteries. J. Power Sources 2016, 328, 615-626. [CrossRef]

280. Malysz, P.; Ye, J.; Gu, R.; Yang, H.; Emadi, A. Battery State-of-Power Peak Current Calculation and Verification Using an Asymmetric Parameter Equivalent Circuit Model. IEEE Trans. Veh. Technol. 2016, 65, 4512-4522. [CrossRef]

281. Lelie, M.; Braun, T.; Knips, M.; Nordmann, H.; Ringbeck, F.; Zappen, H.; Sauer, D. Battery Management System Hardware Concepts: An Overview. Appl. Sci. 2018, 8, 534. [CrossRef]

282. Luca, R.; Whiteley, M.; Neville, T.; Tranter, T.; Weaving, J.; Marco, J.; Shearing, P.R.; Brett, D.J.L. Current Imbalance in Parallel Battery Strings Measured Using a Hall-Effect Sensor Array. Energy Technol. 2021, 9, 2001014. [CrossRef]

283. Schneider, D.; Vögele, U.; Endisch, C. Model-based sensor data fusion of quasi-redundant voltage and current measurements in a lithium-ion battery module. J. Power Sources 2019, 440, 227156. [CrossRef]

284. Schneider, D.; Endisch, C. Robustness and Reliability of Model-based Sensor Data Fusion in a Lithium-Ion Battery System. In Proceedings of the 2020 IEEE Conference on Control Technology and Applications (CCTA), Montreal, QC, Canada, 24-26 August 2020. [CrossRef]

285. Ripka, P. Contactless measurement of electric current using magnetic sensors. tm-Tech. Mess. 2019, 86, 586-598. [CrossRef]

286. Ziegler, S.; Woodward, R.C.; Iu, H.H.-C.; Borle, L.J. Current Sensing Techniques: A Review. IEEE Sens. J. 2009, 86, 354-376. [CrossRef]

287. Patel, A.; Ferdowsi, M. Current Sensing for Automotive Electronics-A Survey. IEEE Trans. Veh. Technol. 2009, 58, 4108-4119. [CrossRef]

288. Takaya, H.; Miyagi, K.; Hamada, K. Current-sensing power MOSFETs with excellent temperature characteristics. In Proceedings of the 2009 21st International Symposium on Power Semiconductor Devices \& IC's, Barcelona, Spain, 14-18 June 2009; pp. 73-76. [CrossRef]

289. Meddings, N.; Heinrich, M.; Overney, F.; Lee, J.S.; Ruiz, V.; Napolitano, E.; Seitz, S.; Hinds, G.; Raccichini, R.; Gaberšček, M.; et al. Application of electrochemical impedance spectroscopy to commercial Li-ion cells: A review. J. Power Sources 2020, 480, 228742. [CrossRef] 
290. Gong, Z.; Liu, Z.; Wang, Y.; Gupta, K.; da Silva, C.; Liu, T.; Zheng, Z.H.; Zhang, W.P.; van Lammeren, J.P.M.; Bergveld, H.J.; et al. IC for online EIS in automotive batteries and hybrid architecture for high-current perturbation in low-impedance cells. In Proceedings of the APEC 2018, San Antonio, TX, USA, 4-8 March 2018; IEEE: Piscataway, NJ, USA, 2018; pp. 1922-1929. [CrossRef]

291. Alavi, S.; Birkl, C.R.; Howey, D.A. Time-domain fitting of battery electrochemical impedance models. J. Power Sources 2015, 288, 345-352. [CrossRef]

292. Depernet, D.; Ba, O.; Berthon, A. Online impedance spectroscopy of lead acid batteries for storage management of a standalone power plant. J. Power Sources 2012, 219, 65-74. [CrossRef]

293. Liebhart, B.; Diehl, S.; Schneider, D.; Endisch, C.; Kennel, R. Enhancing the Cell Impedance Estimation of a Lithium-Ion Battery System with Embedded Power Path Switches. In Proceedings of the 2021 IEEE Applied Power Electronics Conference and Exposition (APEC), Phoenix, AZ, USA, 14-17 June 2021; pp. 967-974. [CrossRef]

294. Liebhart, B.; Diehl, S.; Schmid, M.; Endisch, C.; Kennel, R. Improved Impedance Measurements for Electric Vehicles with Reconfigurable Battery Systems. In Proceedings of the 2021 IEEE 12th Energy Conversion Congress \& Exposition-Asia (ECCEAsia), Singapore, 24-27 May 2021; pp. 1736-1742. [CrossRef]

295. Sihvo, J.; Messo, T.; Roinila, T.; Luhtala, R. Online Internal Impedance Measurements of Li-ion Battery Using PRBS Broadband Excitation and Fourier Techniques: Methods and Injection Design. In Power Electronics for Sustainable Society; IEEE: Piscataway, NJ, USA, 2018; pp. 2470-2475. [CrossRef]

296. Din, E.; Schaef, C.; Moffat, K.; Stauth, J.T. A Scalable Active Battery Management System With Embedded Real-Time Electrochemical Impedance Spectroscopy. IEEE Trans. Power Electron. 2017, 32, 5688-5698. [CrossRef]

297. Piret, H.; Portier, B.; Bacquet, S.; Palmieri, M.; Granjon, P.; Guillet, N.; Cattin, V. Key parameters design for online battery electrochemical impedance tracker. In Proceedings of the European Battery, Hybrid and Fuel Cell Electric Vehicle Congress, Brussels, Belgium, 1-4 December 2015.

298. Yao, Q.; Lu, D.; Lei, G. Battery Impedance Measurement Using Fast Square Current Perturbation. In Proceedings of the 2019 IEEE 4th International Future Energy Electronics Conference (IFEEC), Singapore, 25-28 November 2019; pp. 1-5. [CrossRef]

299. Koch, R.; Kuhn, R.; Zilberman, I.; Jossen, A. Electrochemical impedance spectroscopy for online battery monitoring-power electronics control. In Proceedings of the 2014 16th European Conference on Power Electronics and Applications, Lappeenranta, Finland, 26-28 August 2014; pp. 1-10. [CrossRef]

300. Nguyen, T.T.; Doan, V.T.; Lee, G.H.; Kim, H.W.; Choi, W.; Kim, D.W. Development of an Intelligent Charger with a Battery Diagnosis Function Using Online Impedance Spectroscopy. J. Power Electron. 2016, 16, 1981-1989. [CrossRef]

301. Ferrero, R.; Wu, C.; Carboni, A.; Toscani, S.; de Angelis, M.; George-Williams, H.; Patelli, E.; Pegoraro, P.A. Low-Cost Battery Monitoring by Converter-Based Electrochemical Impedance Spectroscopy. In Proceedings of the 2017 IEEE International Workshop on Applied Measurements for Power Systems (AMPS), Liverpool, UK, 20-22 September 2017; pp. 1-6. [CrossRef]

302. Moore, S.; Barendse, P. Online condition monitoring of lithium-ion batteries using impedance spectroscopy. In Proceedings of the ECCE 2017, Cincinnati, OH, USA, 1-5 October 2017; IEEE: Piscataway, NJ, USA, 2017; pp. 5617-5624. [CrossRef]

303. Hong, P.; Jiang, H.; Li, J.q.; Xu, L.; Ouyang, M. Modeling and Experiment Validation of the DC/DC Converter for Online AC Impedance Identification of the Lithium-Ion Battery. SAE Int. J. Altern. Powertrains 2017, 6, 233-245. [CrossRef]

304. Qahouq, J.A.A.; Xia, Z. Single-Perturbation-Cycle Online Battery Impedance Spectrum Measurement Method With Closed-Loop Control of Power Converter. IEEE Trans. Ind. Electron. 2017, 64, 7019-7029. [CrossRef]

305. Piret, H.; Sockeel, N.; Heiries, V.; Michel, P.H.; Ranieri, M.; Cattin, V.; Guillet, N.; Granjon, P. Passive and active tracking of electrochemical impedance of a drone battery. In Proceedings of the European Battery, Hybrid and Fuel Cell Electric Vehicle Congress, Brussels, Belgium, 1-4 December 2015.

306. Sockeel, N.; Ball, J.; Shahverdi, M.; Mazzola, M. Passive Tracking of the Electrochemical Impedance of a Hybrid Electric Vehicle Battery and State of Charge Estimation through an Extended and Unscented Kalman Filter. Batteries 2018, 4, 52. [CrossRef]

307. Lohmann, N.; Haussmann, P.; Wesskamp, P.; Melbert, J.; Musch, T. Employing Real Automotive Driving Data for Electrochemical Impedance Spectroscopy on Lithium-Ion Cells. SAE Int. J. Altern. Powertrains 2015, 4, 308-317. [CrossRef]

308. Lohmann, N.; Weßkamp, P.; Haußmann, P.; Melbert, J.; Musch, T. Electrochemical impedance spectroscopy for lithium-ion cells: Test equipment and procedures for aging and fast characterization in time and frequency domain. J. Power Sources 2015, 273, 613-623. [CrossRef]

309. Mingant, R.; Bernard, J.; Sauvant-Moynot, V. Towards Onboard Li-ion Battery State-of-health Diagnosis by a Virtual Sensor. World Electr. Veh. J. 2012, 5, 405-411. [CrossRef]

310. Roscher, V.; Schneider, M.; Durdaut, P.; Sassano, N.; Pereguda, S.; Mense, E.; Riemschneider, K.R. Synchronisation using wireless trigger-broadcast for impedance spectroscopy of battery cells. In Proceedings of the 2015 IEEE Sensors Applications Symposium (SAS), Zadar, Croatia, 13-15 April 2015; IEEE: Piscataway, NJ, USA, 2015; pp. 1-6. [CrossRef]

311. Wei, X.; Wang, X.; Dai, H. Practical On-Board Measurement of Lithium Ion Battery Impedance Based on Distributed Voltage and Current Sampling. Energies 2018, 11, 64. [CrossRef]

312. Zhang, L.; Zhou, Z.; Chen, Q.; Long, R.; Quan, S. Model Predictive Control for Electrochemical Impedance Spectroscopy Measurement of Fuel Cells Based on Neural Network Optimization. IEEE Trans. Transp. Electrif. 2019, 5, 524-534. [CrossRef]

313. Ivanisevic, N.; Rodriguez, S.; Rusu, A. Impedance Spectroscopy Based on Linear System Identification. IEEE Trans. Biomed. Circuits Syst. 2019, 13, 396-402. [CrossRef] 
314. Kuipers, M.; Schröer, P.; Nemeth, T.; Zappen, H.; Blömeke, A.; Sauer, D.U. An Algorithm for an Online Electrochemical Impedance Spectroscopy and Battery Parameter Estimation: Development, Verification and Validation. J. Energy Storage 2020, $30,101517$. [CrossRef]

315. Piret, H.; Granjon, P.; Guillet, N.; Cattin, V. Tracking of electrochemical impedance of batteries. J. Power Sources 2016, 312, 60-69. [CrossRef]

316. Zappen, H.; Ringbeck, F.; Sauer, D. Application of Time-Resolved Multi-Sine Impedance Spectroscopy for Lithium-Ion Battery Characterization. Batteries 2018, 4, 64. [CrossRef]

317. Schmidt, J.P.; Ivers-Tiffée, E. Pulse-fitting-A novel method for the evaluation of pulse measurements, demonstrated for the low frequency behavior of lithium-ion cells. J. Power Sources 2016, 315, 316-323. [CrossRef]

318. Barsoukov, E.; Ryu, S.H.; Lee, H. A novel impedance spectrometer based on carrier function Laplace-transform of the response to arbitrary excitation. J. Electroanal. Chem. 2002, 536, 109-122. [CrossRef]

319. Klotz, D.; Schönleber, M.; Schmidt, J.P.; Ivers-Tiffée, E. New approach for the calculation of impedance spectra out of time domain data. Electrochim. Acta 2011, 56, 8763-8769. [CrossRef]

320. Yokoshima, T.; Mukoyama, D.; Nara, H.; Maeda, S.; Nakazawa, K.; Momma, T.; Osaka, T. Impedance Measurements of Kilowatt-Class Lithium Ion Battery Modules/Cubicles in Energy Storage Systems by Square-Current Electrochemical Impedance Spectroscopy. Electrochim. Acta 2017, 246, 800-811. [CrossRef]

321. Carbonnier, H.; Barde, H.; Riga, L.; Carre, A. Electrochemical Impedance Spectroscopy for Online Satellite Battery Monitoring Using Square Wave Excitation. In Proceedings of the 2019 European Space Power Conference (ESPC), Juan-les-Pins, France, 30 September-4 October 2019; pp. 1-6. [CrossRef]

322. Osaka, T.; Naoi, K. Application of On-line Impedance Measurement Using Fast Fourier Transform to Electrochemical Systems. Bull. Chem. Soc. Jpn. 1982, 55, 36-40. [CrossRef]

323. Kamat, R.K.; Naik, G.M. Thermistors-In search of new applications, manufacturers cultivate advanced NTC techniques. Sens. Rev. 2002, 22, 334-340. [CrossRef]

324. Cao, J.; Emadi, A. Batteries Need Electronics. IEEE Ind. Electron. Mag. 2011, 5, 27-35. [CrossRef]

325. Anthony, D.; Wong, D.; Wetz, D.; Jain, A. Non-invasive measurement of internal temperature of a cylindrical Li-ion cell during high-rate discharge. Int. J. Heat Mass Transf. 2017, 111, 223-231. [CrossRef]

326. Drake, S.J.; Martin, M.; Wetz, D.A.; Ostanek, J.K.; Miller, S.P.; Heinzel, J.M.; Jain, A. Heat generation rate measurement in a Li-ion cell at large C-rates through temperature and heat flux measurements. J. Power Sources 2015, 285, 266-273. [CrossRef]

327. Childs, P.R.N.; Greenwood, J.R.; Long, C.A. Review of temperature measurement. Rev. Sci. Instrum. 2000, 71, 2959-2978. [CrossRef]

328. Wang, S.; Li, K.; Tian, Y.; Wang, J.; Wu, Y.; Ji, S. Infrared imaging investigation of temperature fluctuation and spatial distribution for a large laminated lithium-ion power battery. Appl. Therm. Eng. 2019, 152, 204-214. [CrossRef]

329. White, D.R.; Galleano, R.; Actis, A.; Brixy, H.; de Groot, M.; Dubbeldam, J.; Reesink, A.L.; Edler, F.; Sakurai, H.; Shepard, R.L.; et al. The status of Johnson noise thermometry. Metrologia 1996, 33, 325-335. [CrossRef]

330. Liu, X.; Zheng, C.; Liu, C.; Pong, P.W.T. Experimental Investigation of a Johnson Noise Thermometry Using GMR Sensor for Electric Vehicle Applications. IEEE Sens. J. 2018, 18, 3098-3107. [CrossRef]

331. Fleming, J.; Amietszajew, T.; Charmet, J.; Roberts, A.J.; Greenwood, D.; Bhagat, R. The design and impact of in-situ and operando thermal sensing for smart energy storage. J. Energy Storage 2019, 22, 36-43. [CrossRef]

332. Zhang, G.; Cao, L.; Ge, S.; Wang, C.Y.; Shaffer, C.E.; Rahn, C.D. Reaction temperature sensing (RTS)-based control for Li-ion battery safety. Sci. Rep. 2015, 5, 18237. [CrossRef]

333. Zhu, S.; Han, J.; An, H.Y.; Pan, T.S.; Wei, Y.M.; Song, W.L.; Chen, H.S.; Fang, D. A novel embedded method for in-situ measuring internal multi-point temperatures of lithium ion batteries. J. Power Sources 2020, 456, 227981. [CrossRef]

334. Mutyala, M.S.K.; Zhao, J.; Li, J.; Pan, H.; Yuan, C.; Li, X. In-situ temperature measurement in lithium ion battery by transferable flexible thin film thermocouples. J. Power Sources 2014, 260, 43-49. [CrossRef]

335. Martiny, N.; Rheinfeld, A.; Geder, J.; Wang, Y.; Kraus, W.; Jossen, A. Development of an All Kapton-Based Thin-Film Thermocouple Matrix for In Situ Temperature Measurement in a Lithium Ion Pouch Cell. IEEE Sens. J. 2014, 14, 3377-3384. [CrossRef]

336. Raghavan, A.; Kiesel, P.; Sommer, L.W.; Schwartz, J.; Lochbaum, A.; Hegyi, A.; Schuh, A.; Arakaki, K.; Saha, B.; Ganguli, A.; et al. Embedded fiber-optic sensing for accurate internal monitoring of cell state in advanced battery management systems part 1: Cell embedding method and performance. J. Power Sources 2017, 341, 466-473. [CrossRef]

337. Martiny, N.; Geder, J.; Wang, Y.; Kraus, W.; Jossen, A. Development of a thin-film thermocouple matrix for in-situ temperature measurement in a lithium ion pouch cell. In Proceedings of the IEEE Sensors, Baltimore, MD, USA, 3-6 November 2013; IEEE: Piscataway, NJ, USA, 2013; pp. 1-4. [CrossRef]

338. Charmet, J.; Bitterli, J.; Sereda, O.; Liley, M.; Renaud, P.; Keppner, H. Optimizing Parylene C Adhesion for MEMS Processes: Potassium Hydroxide Wet Etching. J. Microelectromech. Syst. 2013, 22, 855-864. [CrossRef]

339. Oh, K.Y.; Epureanu, B.I. A novel thermal swelling model for a rechargeable lithium-ion battery cell. J. Power Sources 2016, 303, 86-96. [CrossRef]

340. Oh, K.Y.; Epureanu, B.I.; Siegel, J.B.; Stefanopoulou, A.G. Phenomenological force and swelling models for rechargeable lithium-ion battery cells. J. Power Sources 2016, 310, 118-129. [CrossRef] 
341. Rieger, B.; Schlueter, S.; Erhard, S.V.; Schmalz, J.; Reinhart, G.; Jossen, A. Multi-scale investigation of thickness changes in a commercial pouch type lithium-ion battery. J. Energy Storage 2016, 6, 213-221. [CrossRef]

342. Kim, Y.; Mohan, S.; Samad, N.A.; Siegel, J.B.; Stefanopoulou, A.G. Optimal power management for a series hybrid electric vehicle cognizant of battery mechanical effects. In Proceedings of the 2014 American Control Conference, Portland, OR, USA, 4-6 June 2014; pp. 3832-3837. [CrossRef]

343. Popp, H.; Koller, M.; Jahn, M.; Bergmann, A. Mechanical methods for state determination of Lithium-Ion secondary batteries: A review. J. Energy Storage 2020, 32, 101859. [CrossRef]

344. Cheng, X.; Pecht, M. In Situ stress measurement techniques on li-ion battery electrodes: A review. Energies 2017, $10,591$. [CrossRef]

345. Knobloch, A.; Kapusta, C.; Karp, J.; Plotnikov, Y.; Siegel, J.B.; Stefanopoulou, A.G. Fabrication of Multimeasurand Sensor for Monitoring of a Li-Ion Battery. J. Electron. Packag. 2018, 140, 031002. [CrossRef]

346. Knobloch, A.; Karp, J.; Plotnikov, Y.; Kapusta, C.; Siegel, J.; Samad, N.; Stefanopoulou, A. Novel thin temperature and expansion sensors for li-ion battery monitoring. In Proceedings of the 2017 IEEE SENSORS, Glasgow, UK, 29 October-1 November 2017; pp. 1-3. [CrossRef]

347. Kinet, D.; Mégret, P.; Goossen, K.W.; Qiu, L.; Heider, D.; Caucheteur, C. Fiber Bragg grating sensors toward structural health monitoring in composite materials: Challenges and solutions. Sensors 2014, 14, 7394-7419. [CrossRef]

348. Fleming, J.; Amietszajew, T.; McTurk, E.; Towers, D.P.; Greenwood, D.; Bhagat, R. Development and evaluation of in-situ instrumentation for cylindrical Li-ion cells using fibre optic sensors. HardwareX 2018, 3, 100-109. [CrossRef]

349. McTurk, E.; Amietszajew, T.; Fleming, J.; Bhagat, R. Thermo-electrochemical instrumentation of cylindrical Li-ion cells. J. Power Sources 2018, 379, 309-316. [CrossRef]

350. Amietszajew, T.; McTurk, E.; Fleming, J.; Bhagat, R. Understanding the limits of rapid charging using instrumented commercial 18650 high-energy Li-ion cells. Electrochim. Acta 2018, 263, 346-352. [CrossRef]

351. Finegan, D.P.; Darcy, E.; Keyser, M.; Tjaden, B.; Heenan, T.M.M.; Jervis, R.; Bailey, J.J.; Vo, N.T.; Magdysyuk, O.V.; Drakopoulos, M.; et al. Identifying the Cause of Rupture of Li-Ion Batteries during Thermal Runaway. Adv. Sci. 2018, 5, 1700369. [CrossRef]

352. Nascimento, M.; Ferreira, M.; Pinto, J. Simultaneous Sensing of Temperature and Bi-Directional Strain in a Prismatic Li-Ion Battery. Batteries 2018, 4, 23. [CrossRef]

353. Nascimento, M.; Novais, S.; Ding, M.S.; Ferreira, M.S.; Koch, S.; Passerini, S.; Pinto, J.L. Internal strain and temperature discrimination with optical fiber hybrid sensors in Li-ion batteries. J. Power Sources 2019, 410-411, 1-9. [CrossRef] [PubMed]

354. Peng, J.; Zhou, X.; Jia, S.; Jin, Y.; Xu, S.; Chen, J. High precision strain monitoring for lithium ion batteries based on fiber Bragg grating sensors. J. Power Sources 2019, 433, 226692. [CrossRef]

355. Nascimento, M.; Ferreira, M.S.; Pinto, J.L. Real time thermal monitoring of lithium batteries with fiber sensors and thermocouples: A comparative study. Measurement 2017, 111, 260-263. [CrossRef]

356. Bae, C.J.; Manandhar, A.; Kiesel, P.; Raghavan, A. Monitoring the Strain Evolution of Lithium-Ion Battery Electrodes using an Optical Fiber Bragg Grating Sensor. Energy Technol. 2016, 4, 851-855. [CrossRef]

357. Nedjalkov, A.; Meyer, J.; Gräfenstein, A.; Schramm, B.; Angelmahr, M.; Schwenzel, J.; Schade, W. Refractive Index Measurement of Lithium Ion Battery Electrolyte with Etched Surface Cladding Waveguide Bragg Gratings and Cell Electrode State Monitoring by Optical Strain Sensors. Batteries 2019, 5, 30. [CrossRef]

358. Fortier, A.; Tsao, M.; Williard, N.; Xing, Y.; Pecht, M. Preliminary Study on Integration of Fiber Optic Bragg Grating Sensors in Li-Ion Batteries and In Situ Strain and Temperature Monitoring of Battery Cells. Energies 2017, 10, 838. [CrossRef]

359. Novais, S.; Nascimento, M.; Grande, L.; Domingues, M.F.; Antunes, P.; Alberto, N.; Leitão, C.; Oliveira, R.; Koch, S.; Kim, G.T.; et al. Internal and External Temperature Monitoring of a Li-Ion Battery with Fiber Bragg Grating Sensors. Sensors 2016, 16, 1394. [CrossRef]

360. Ghannoum, A.; Iyer, K.; Nieva, P.; Khajepour, A. Fiber optic monitoring of lithium-ion batteries: A novel tool to understand the lithiation of batteries. In Proceedings of the IEEE SENSORS 2016, Orlando, FL, USA, 30 October-3 November 2016; IEEE: Piscataway, NJ, USA, 2016; pp. 1-3. [CrossRef]

361. Ghannoum, A.; Nieva, P. Graphite lithiation and capacity fade monitoring of lithium ion batteries using optical fibers. J. Energy Storage 2020, 28, 101233. [CrossRef]

362. Yang, L.; Chen, H.S.; Song, W.L.; Fang, D. Effect of Defects on Diffusion Behaviors of Lithium-Ion Battery Electrodes: In Situ Optical Observation and Simulation. ACS Appl. Mater. Interfaces 2018, 10, 43623-43630. [CrossRef]

363. Davies, G.; Knehr, K.W.; van Tassell, B.; Hodson, T.; Biswas, S.; Hsieh, A.G.; Steingart, D.A. State of charge and state of health estimation using electrochemical acoustic time of flight analysis. J. Electrochem. Soc. 2017, 164, A2746. [CrossRef]

364. Hsieh, A.G.; Bhadra, S.; Hertzberg, B.J.; Gjeltema, P.J.; Goy, A.; Fleischer, J.W.; Steingart, D.A. Electrochemical-acoustic time of flight: In operando correlation of physical dynamics with battery charge and health. Energy Environ. Sci. 2015, 8, 1569-1577. [CrossRef]

365. Robinson, J.B.; Maier, M.; Alster, G.; Compton, T.; Brett, D.J.L.; Shearing, P.R. Spatially resolved ultrasound diagnostics of Li-ion battery electrodes. Phys. Chem. Chem. Phys. 2019, 21, 6354-6361. [CrossRef]

366. Gold, L.; Bach, T.; Virsik, W.; Schmitt, A.; Müller, J.; Staab, T.E.; Sextl, G. Probing lithium-ion batteries' state-of-charge using ultrasonic transmission-Concept and laboratory testing. J. Power Sources 2017, 343, 536-544. [CrossRef] 
367. Robinson, J.B.; Pham, M.; Kok, M.D.; Heenan, T.M.; Brett, D.J.; Shearing, P.R. Examining the Cycling Behaviour of Li-Ion Batteries Using Ultrasonic Time-of-Flight Measurements. J. Power Sources 2019, 444, 227318. [CrossRef]

368. Sood, B.; Osterman, M.; Pecht, M. Health monitoring of lithium-ion batteries. In Proceedings of the 10th Annual IEEE Symposium on Product Compliance Engineering ISPCE, Austin, TX, USA, 7-9 October 2013; pp. 1-6. [CrossRef]

369. Zappen, H.; Fuchs, G.; Gitis, A.; Sauer, D.U. In-Operando Impedance Spectroscopy and Ultrasonic Measurements during High-Temperature Abuse Experiments on Lithium-Ion Batteries. Batteries 2020, 6, 25. [CrossRef]

370. Ladpli, P.; Kopsaftopoulos, F.; Chang, F.K. Estimating state of charge and health of lithium-ion batteries with guided waves using built-in piezoelectric sensors/actuators. J. Power Sources 2018, 384, 342-354. [CrossRef]

371. Chang, J.J.; Zeng, X.F.; Wan, T.L. Real-time measurement of lithium-ion batteries' state-of-charge based on air-coupled ultrasound. AIP Adv. 2019, 9, 085116. [CrossRef]

372. Muhammad, S.; Rafique, M.U.; Li, S.; Shao, Z.; Wang, Q.; Liu, X. Reconfigurable Battery Systems. ACM Trans. Des. Autom. Electron. Syst. 2019, 24, 1-27. [CrossRef]

373. Ci, S.; Lin, N.; Wu, D. Reconfigurable Battery Techniques and Systems: A Survey. IEEE Access 2016, 4, 1175-1189. [CrossRef]

374. Helling, F.; Glück, J.; Singer, A.; Pfisterer, H.J.; Weyh, T. The AC battery-A novel approach for integrating batteries into AC systems. Int. J. Electr. Power Energy Syst. 2019, 104, 150-158. [CrossRef]

375. Kim, T.; Qiao, W.; Qu, L. A series-connected self-reconfigurable multicell battery capable of safe and effective charging/discharging and balancing operations. In Proceedings of the 2012 Twenty-Seventh Annual IEEE Applied Power Electronics Conference and Exposition (APEC), Orlando, FL, USA, 5-9 February 2012; pp. 2259-2264. [CrossRef]

376. He, L.; Yang, Z.; Gu, Y.; Liu, C.; He, T.; Shin, K.G. SoH-Aware Reconfiguration in Battery Packs. IEEE Trans. Smart Grid 2018, 9,3727-3735. [CrossRef]

377. Kim, T.; Qiao, W.; Qu, L. Power Electronics-Enabled Self-X Multicell Batteries: A Design Toward Smart Batteries. IEEE Trans. Power Electron. 2012, 27, 4723-4733. [CrossRef]

378. Kim, T.; Qiao, W.; Qu, L. A multicell battery system design for electric and plug-in hybrid electric vehicles. In Proceedings of the 2012 IEEE International Electric Vehicle Conference, Greenville, SC, USA, 4-8 March 2012; pp. 1-7. [CrossRef]

379. Manenti, A.; Abba, A.; Merati, A.; Savaresi, S.M.; Geraci, A. A New BMS Architecture Based on Cell Redundancy. IEEE Trans. Ind. Electron. 2011, 58, 4314-4322. [CrossRef]

380. Tolbert, L.A.; Peng, F.Z.; Cunnyngham, T.; Chiasson, J.N. Charge balance control schemes for cascade multilevel converter in hybrid electric vehicles. IEEE Trans. Ind. Electron. 2002, 49, 1058-1064. [CrossRef]

381. Zhu, Q.; Dai, W.; Guan, L.; Tan, X.; Li, Z.; Xie, D. A Fault-Tolerant Control Strategy of Modular Multilevel Converter with Sub-Module Faults Based on Neutral Point Compound Shift. Energies 2019, 12, 876. [CrossRef]

382. Venkataramanaiah, J.; Suresh, Y.; Panda, A.K. A review on symmetric, asymmetric, hybrid and single DC sources based multilevel inverter topologies. Renew. Sustain. Energy Rev. 2017, 76, 788-812. [CrossRef]

383. Chen, Z.; Lin, F.; Wang, C.; Le Wang, Y.; Xu, M. Active Diagnosability of Discrete Event Systems and its Application to Battery Fault Diagnosis. IEEE Trans. Control Syst. Technol. 2014, 22, 1892-1898. [CrossRef]

384. Lezana, P.; Pou, J.; Meynard, T.A.; Rodriguez, J.; Ceballos, S.; Richardeau, F. Survey on Fault Operation on Multilevel Inverters. IEEE Trans. Ind. Electron. 2010, 57, 2207-2218. [CrossRef]

385. Maharjan, L.; Yamagishi, T.; Akagi, H.; Asakura, J. Fault-tolerant control for a battery energy storage system based on a cascade PWM converter. In Proceedings of the IEEE 6th International Power Electronics and Motion Control Conference, Wuhan, China, 17-20 May 2009; IEEE: Piscataway, NJ, USA, 2009; pp. 945-950. [CrossRef]

386. Kleiner, J.; Lechermann, L.; Komsiyska, L.; Elger, G.; Endisch, C. Thermal Effects of Bad-Block-Management in an Intelligent Automotive Lithium-ion Battery Module based on lumped 3D Electro-Thermal Modeling. In Proceedings of the 2021 IEEE Transportation Electrification Conference \& Expo (ITEC), Chicago, IL, USA, 21-25 June 2021.

387. Konstantinou, G.; Pou, J.; Ceballos, S.; Agelidis, V.G. Active Redundant Submodule Configuration in Modular Multilevel Converters. IEEE Trans. Power Deliv. 2013, 28, 2333-2341. [CrossRef]

388. Liu, G.; Xu, Z.; Xue, Y.; Tang, G. Optimized Control Strategy Based on Dynamic Redundancy for the Modular Multilevel Converter. IEEE Trans. Power Electron. 2015, 30, 339-348. [CrossRef]

389. Zhang, W.; Xu, D.; Enjeti, P.N.; Li, H.; Hawke, J.T.; Krishnamoorthy, H.S. Survey on Fault-Tolerant Techniques for Power Electronic Converters. IEEE Trans. Power Electron. 2014, 29, 6319-6331. [CrossRef]

390. Hammond, P.W. Enhancing the reliability of modular medium-voltage drives. IEEE Trans. Ind. Electron. 2002, $49,948-954$. [CrossRef]

391. Yu, Y.; Konstantinou, G.; Townsend, C.D.; Agelidis, V.G. Comparison of zero-sequence injection methods in cascaded H-bridge multilevel converters for large-scale photovoltaic integration. IET Renew. Power Gener. 2017, 11, 603-613. [CrossRef]

392. Lezana, P.; Ortiz, G. Extended Operation of Cascade Multicell Converters Under Fault Condition. IEEE Trans. Ind. Electron. 2009, 56, 2697-2703. [CrossRef]

393. Herzog, M.; Ebentheuer, A.W.; Lahlou, T.; Herzog, H.G. Management Algorithms for Cascaded H-Bridge Multilevel Inverters. In Proceedings of the 2018 53rd International Universities Power Engineering Conference (UPEC2018), Glasgow, UK, 4-7 September 2018; pp. 1-6. [CrossRef] 
394. Kim, S.M.; Lee, J.S.; Lee, K.B. Fault-tolerant strategy using neutral-shift method for cascaded multilevel inverters based on level-shifted PWM. In Proceedings of the 2015 9th International Conference on Power Electronics and ECCE Asia (ICPE-ECCE Asia), Seoul, Korea, 1-5 June 2015; IEEE: Piscataway, NJ, USA, 2015; pp. 1327-1332. [CrossRef]

395. Daowd, M.; Omar, N.; van den Bossche, P.; van Mierlo, J. Passive and active battery balancing comparison based on MATLAB simulation. In Proceedings of the IEEE Vehicle Power and Propulsion Conference (VPPC), Chicago, IL, USA, 6-9 September 2011; IEEE: Piscataway, NJ, USA, 2011; pp. 1-7. [CrossRef]

396. Aizpuru, I.; Iraola, U.; Canales, J.M.; Unamuno, E.; Gil, I. Battery pack tests to detect unbalancing effects in series connected Li-ion cells. In Proceedings of the International Conference on Clean Electrical Power (ICCEP), Alghero, Italy, 11-13 June 2013; IEEE: Piscataway, NJ, USA, 2013; pp. 99-106. [CrossRef]

397. Santhanagopalan, S.; White, R.E. Quantifying Cell-to-Cell Variations in Lithium Ion Batteries. Int. J. Electrochem. 2012, 2012, 1-10. [CrossRef]

398. Baumhöfer, T.; Brühl, M.; Rothgang, S.; Sauer, D.U. Production caused variation in capacity aging trend and correlation to initial cell performance. J. Power Sources 2014, 247, 332-338. [CrossRef]

399. Schuster, S.F.; Brand, M.J.; Berg, P.; Gleissenberger, M.; Jossen, A. Lithium-ion cell-to-cell variation during battery electric vehicle operation. J. Power Sources 2015, 297, 242-251. [CrossRef]

400. Gao, Z.C.; Chin, C.S.; Toh, W.D.; Chiew, J.; Jia, J. State-of-Charge Estimation and Active Cell Pack Balancing Design of Lithium Battery Power System for Smart Electric Vehicle. J. Adv. Transp. 2017, 2017, 1-14. [CrossRef]

401. Ricco, M.; Meng, J.; Gherman, T.; Grandi, G.; Teodorescu, R. Smart Battery Pack for Electric Vehicles Based on Active Balancing with Wireless Communication Feedback. Energies 2019, 12, 3862. [CrossRef]

402. Cao, J.; Schofield, N.; Emadi, A. Battery balancing methods: A comprehensive review. In Proceedings of the IEEE Vehicle Power and Propulsion Conference, Harbin, China, 3-5 September 2008; IEEE: Piscataway, NJ, USA, 2008; pp. 1-6. [CrossRef]

403. Qi, J.; Dah-Chuan Lu, D. Review of battery cell balancing techniques. In Proceedings of the Australasian Universities Power Engineering Conference (AUPEC), Perth, WA, Australia, 28 September-1 October 2014; Abu-Siada, A., Ed.; IEEE: Piscataway, NJ, USA, 2014; pp. 1-6. [CrossRef]

404. Omariba, Z.B.; Zhang, L.; Sun, D. Review of Battery Cell Balancing Methodologies for Optimizing Battery Pack Performance in Electric Vehicles. IEEE Access 2019, 7, 129335-129352. [CrossRef]

405. Bouchhima, N.; Schnierle, M.; Schulte, S.; Birke, K.P. Active model-based balancing strategy for self-reconfigurable batteries. J. Power Sources 2016, 322, 129-137. [CrossRef]

406. Alaas, Z.; Wang, C.; Jiang, C.; Duan, C.; Chen, J. Improved cascaded multilevel battery inverters with phase-to-phase SOC balancing capability. In Proceedings of the 2017 IEEE Transportation and Electrification Conference and Expo (ITEC), Chicago, IL, USA, 22-24 June 2017; IEEE: Piscataway, NJ, USA, 2017; pp. 323-327. [CrossRef]

407. Vasiladiotis, M.; Rufer, A. Balancing control actions for cascaded H-bridge converters with integrated battery energy storage. In Proceedings of the 2013 15th European Conference on Power Electronics and Applications (EPE), Lille, France, 2-6 September 2013; pp. 1-10. [CrossRef]

408. Yang, C.Y.; Chen, Y.M.; Juang, K.C. A sorting balance control for battery sources in a single phase multilevel inverter. In Proceedings of the IEEE Energy Conversion Congress and Exposition (ECCE), Cincinnati, OH, USA, 1-5 October 2017; pp. $2742-2747$. [CrossRef]

409. Kim, J.H.; Kang, M.S.; Oh, S.B.; Kim, E.H. New control scheme of lithium-polymer battery units using single phase multi-level converter. In Proceedings of the IEEE 8th International Conference on Power Electronics and ECCE Asia (ICPE \& ECCE), Jeju, Korea, 30 May-3 June 2011; IEEE: Piscataway, NJ, USA, 2011; pp. 2997-3000. [CrossRef]

410. Babaei, E.; Azimpour, Y.; Kangarlu, M.F. Charge balance control of a seven-level asymmetric cascade multilevel inverter. In Proceedings of the 3rd IEEE International Symposium on Power Electronics for Distributed Generation Systems (PEDG), Aalborg, Denmark, 25-28 June 2012; IEEE: Piscataway, NJ, USA, 2012; pp. 674-681. [CrossRef]

411. Chang, F.; Zheng, Z.; Li, Y.; Peng, L. A two-level SOC balance strategy for a novel hybrid energy storage topology. In Proceedings of the 2015 17th European Conference on Power Electronics and Applications (EPE'15 ECCE-Europe), Geneva, Switzerland, 8-10 September 2015; pp. 1-10. [CrossRef]

412. Townsend, C.D.; Summers, T.J.; Betz, R.E. Phase-Shifted Carrier Modulation Techniques for Cascaded H-Bridge Multilevel Converters. IEEE Trans. Ind. Electron. 2015, 62, 6684-6696. [CrossRef]

413. D'Arco, S.; Piegari, L.; Tricoli, P. A modular converter with embedded battery cell balancing for electric vehicles. In Proceedings of the Electrical Systems for Aircraft, Railway and Ship Propulsion (ESARS), Bologna, Italy, 16-18 October 2012; IEEE: Piscataway, NJ, USA, 2012; pp. 1-6. [CrossRef]

414. Ilves, K.; Harnefors, L.; Norrga, S.; Nee, H.P. Predictive Sorting Algorithm for Modular Multilevel Converters Minimizing the Spread in the Submodule Capacitor Voltages. IEEE Trans. Power Electron. 2015, 30, 440-449. [CrossRef]

415. Siemaszko, D. Fast Sorting Method for Balancing Capacitor Voltages in Modular Multilevel Converters. IEEE Trans. Power Electron. 2015, 30, 463-470. [CrossRef]

416. Goetz, S.M.; Li, Z.; Liang, X.; Zhang, C.; Lukic, S.M.; Peterchev, A.V. Control of Modular Multilevel Converter With Parallel Connectivity-Application to Battery Systems. IEEE Trans. Power Electron. 2017, 32, 8381-8392. [CrossRef] 
417. Kandasamy, K.; Vilathgamuwa, M.; Tseng, K.J. Inter-module state-of-charge balancing and fault-tolerant operation of cascaded H-bridge converter using multi-dimensional modulation for electric vehicle application. IET Power Electron. 2015, 8, 1912-1919. [CrossRef]

418. Maharjan, L.; Inoue, S.; Akagi, H.; Asakura, J. State-of-Charge (SOC)-Balancing Control of a Battery Energy Storage System Based on a Cascade PWM Converter. IEEE Trans. Power Electron. 2009, 24, 1628-1636. [CrossRef]

419. Summers, T.J.; Betz, R.E.; Mirzaeva, G. Phase leg voltage balancing of a cascaded H-Bridge converter based STATCOM using zero sequence injection. In Proceedings of the 2009 13th European Conference on Power Electronics and Applications, Barcelona, Spain, 8-10 September 2009; pp. 1-10.

420. Li, N.; Gao, F.; Hao, T.; Ma, Z.; Zhang, C. SOH Balancing Control Method for the MMC Battery Energy Storage System. IEEE Trans. Ind. Electron. 2018, 65, 6581-6591. [CrossRef]

421. Altaf, F.; Johannesson, L.; Egardt, B. Simultaneous Thermal and State-of-Charge Balancing of Batteries: A Review. In Proceedings of the 2014 IEEE Vehicle Power and Propulsion Conference (VPPC), Coimbra, Portugal, 27-30 October 2014; IEEE: Piscataway, NJ, USA, 2014; pp. 1-7. [CrossRef]

422. Docimo, D.J.; Fathy, H.K. Using a Linear Quadratic Regulator to Attenuate Cell-to-Cell Heterogeneity within a Lithium-Ion Battery Pack. In Proceedings of the 2018 IEEE Conference on Control Technology and Applications (CCTA), Copenhagen, Denmark, 21-24 August 2018; pp. 1395-1402. [CrossRef]

423. Bouchhima, N.; Gossen, M.; Schulte, S.; Birke, K.P. Lifetime of self-reconfigurable batteries compared with conventional batteries. J. Energy Storage 2018, 15, 400-407. [CrossRef]

424. Kleiner, J.; Lechermann, L.; Komsiyska, L.; Elger, G.; Endisch, C. Thermal Behavior of Intelligent Automotive Lithium-Ion Batteries: Operating Strategies for Adaptive Thermal Balancing by Reconfiguration. J. Energy Storage 2021, 40, 102686. [CrossRef]

425. Altai, F.; Egardt, B.; Johannesson, L. Electro-thermal Control of Modular Battery using Model Predictive Control with Control Projections. IFAC-PapersOnLine 2015, 48, 368-375. [CrossRef]

426. Hasan, N.S.; Rosmin, N.; Osman, D.A.A.; Hatib, A. Reviews on multilevel converter and modulation techniques. Renew. Sustain. Energy Rev. 2017, 80, 163-174. [CrossRef]

427. Kamaldeep; Kumar, J. Switch reduction and performance analysis using different modulation technique in multilevel inverter. In Proceedings of the First IEEE International Conference on Power Electronics, Intelligent Control and Energy Systems (IPEICES 2016), Delhi, India, 4-6 July 2016; pp. 1-4. [CrossRef]

428. Hinago, Y.; Koizumi, H. A Switched-Capacitor Inverter Using Series/Parallel Conversion With Inductive Load. IEEE Trans. Ind. Electron. 2012, 59, 878-887. [CrossRef]

429. Nabae, A.; Takahashi, I.; Akagi, H. A New Neutral-Point-Clamped PWM Inverter. IEEE Trans. Ind. Appl. 1981, IA-17, 518-523. [CrossRef]

430. Rodriguez, J.; Bernet, S.; Wu, B.; Pontt, J.O.; Kouro, S. Multilevel Voltage-Source-Converter Topologies for Industrial MediumVoltage Drives. IEEE Trans. Ind. Electron. 2007, 54, 2930-2945. [CrossRef]

431. Goetz, S.M.; Peterchev, A.V.; Weyh, T. Modular Multilevel Converter With Series and Parallel Module Connectivity: Topology and Control. IEEE Trans. Power Electron. 2015, 30, 203-215. [CrossRef]

432. Hinago, Y.; Koizumi, H. A single phase multilevel inverter using switched series/parallel DC voltage sources. In Proceedings of the IEEE Energy Conversion Congress and Exposition, San Jose, CA, USA, 20-24 September 2009; IEEE: Piscataway, NJ, USA, 2009; pp. 1962-1967. [CrossRef]

433. Vasiladiotis, M.; Rufer, A. Analysis and Control of Modular Multilevel Converters With Integrated Battery Energy Storage. IEEE Trans. Power Electron. 2015, 30, 163-175. [CrossRef]

434. Quraan, M.; Tricoli, P.; D Arco, S.; Piegari, L. Efficiency Assessment of Modular Multilevel Converters for Battery Electric Vehicles. IEEE Trans. Power Electron. 2017, 32, 2041-2051. [CrossRef]

435. Korte, C.; Specht, E.; Hiller, M.; Goetz, S. Efficiency evaluation of MMSPC/CHB topologies for automotive applications. In Proceedings of the 2017 IEEE 12th International Conference on Power Electronics and Drive Systems (PEDS), Honolulu, HI, USA, 12-15 December 2017; pp. 324-330. [CrossRef]

436. Baronti, F.; Fantechi, G.; Roncella, R.; Saletti, R. Design of a module switch for battery pack reconfiguration in high-power applications. In Proceedings of the 2012 IEEE International Symposium on Industrial Electronics, Hangzhou, China, 28-31 May 2012; pp. 1330-1335. [CrossRef]

437. Babaei, E.; Hosseini, S.H. New cascaded multilevel inverter topology with minimum number of switches. Energy Convers. Manag. 2009, 50, 2761-2767. [CrossRef]

438. Lesnicar, A.; Marquardt, R. An innovative modular multilevel converter topology suitable for a wide power range. In Proceedings of the 2003 IEEE Bologna PowerTech, Bologna, Italy, 23-26 June 2003; pp. 272-277. [CrossRef]

439. Allebrod, S.; Hamerski, R.; Marquardt, R. New transformerless, scalable Modular Multilevel Converters for HVDC-transmission In Proceedings of the IEEE Power Electronics Specialists Conference, Rhodes, Greece, 15-19 June 2008; pp. 174-179. [CrossRef]

440. Alaas, Z.; Wang, C.; Jiang, C.; Duan, C.; Le Wang, Y. A Hierarchal Cascaded Multilevel Converter for uniform SOC battery management. In Proceedings of the 2016 IEEE Transportation Electrification Conference and Expo (ITEC), Dearborn, MI, USA, 27-29 June 2016; pp. 1-6. [CrossRef] 
441. Liu, L.; Gotting, G.; Xie, J. Torque Ripple Reduction Using Variable DC-link Voltage Technique for Permanent Magnet Synchronous Motor in Battery Electric Vehicle. In Proceedings of the 2020 IEEE 29th International Symposium on Industrial Electronics (ISIE), Delft, The Netherlands, 17-19 June 2020; pp. 374-379. [CrossRef]

442. Horsche, M.; Sturm, M.; Jossen, A. Realising Serial Hybrid Energy Storage Systems (sHESS) by Implementing Switching Circuits on Battery Cell Level. World Electr. Veh. J. 2016, 8, 371-378. [CrossRef]

443. D'Arco, S.; Quraan, M.; Tricoli, P.; Piegari, L. Low frequency operation of Modular Multilevel Converters with embedded battery cells for traction drives. In Proceedings of the 2016 International Symposium on Power Electronics, Electrical Drives, Automation and Motion (SPEEDAM), Capri, Italy, 22-24 June 2016; pp. 1375-1382. [CrossRef]

444. Franquelo, L.; Rodriguez, J.; Leon, J.; Kouro, S.; Portillo, R.; Prats, M. The age of multilevel converters arrives. IEEE Ind. Electron. Mag. 2008, 2, 28-39. [CrossRef]

445. McGrath, B.P.; Teixeira, C.A.; Holmes, D.G. Optimized Phase Disposition (PD) Modulation of a Modular Multilevel Converter. IEEE Trans. Ind. Appl. 2017, 53, 4624-4633. [CrossRef]

446. McGrath, B.P.; Holmes, D.G. Multicarrier PWM strategies for multilevel inverters. IEEE Trans. Ind. Electron. 2002, 49, 858-867. [CrossRef]

447. Choi, W.K.; Kang, F.S. H-bridge based multilevel inverter using PWM switching function. In Proceedings of the 31st International Telecommunications Energy Conference, Incheon, Korea, 18-22 October 2009; IEEE: Piscataway, NJ, USA, 2009; pp. 1-5. [CrossRef]

448. Babaei, E.; Farhadi Kangarlu, M. Cross-switched multilevel inverter: An innovative topology. IET Power Electron. 2013, 6, 642-651. [CrossRef]

449. Sabahi, M.; Babaei, E.; Farhadi Kangarlu, M. Cascaded cross-switched multilevel inverter in symmetric and asymmetric conditions. IET Power Electron. 2013, 6, 1041-1050. [CrossRef]

450. Babaei, E.; Hosseini, S.H.; Gharehpetian, G.B.; Haque, M.T.; Sabahi, M. Reduction of de voltage sources and switches in asymmetrical multilevel converters using a novel topology. Electr. Power Syst. Res. 2007, 77, 1073-1085. [CrossRef]

451. Hammond, P.W. A new approach to enhance power quality for medium voltage AC drives. IEEE Trans. Ind. Appl. 1997, 33, 202-208. [CrossRef]

452. Josefsson, O.; Thiringer, T.; Lundmark, S.; Zelaya, H. Evaluation and comparison of a two-level and a multilevel inverter for an EV using a modulized battery topology. In Proceedings of the IECON 2012, Montreal, QC, Canada, 25-28 October 2012; IEEE: Piscataway, NJ, USA, 2012; pp. 2949-2956. [CrossRef]

453. Diong, B.; Sepahvand, H.; Corzine, K.A. Harmonic Distortion Optimization of Cascaded H-Bridge Inverters Considering Device Voltage Drops and Noninteger DC Voltage Ratios. IEEE Trans. Ind. Electron. 2013, 60, 3106-3114. [CrossRef]

454. Routray, A.; Kumar Singh, R.; Mahanty, R. Harmonic Minimization in Three-Phase Hybrid Cascaded Multilevel Inverter Using Modified Particle Swarm Optimization. IEEE Trans. Ind. Inform. 2019, 15, 4407-4417. [CrossRef]

455. Kuder, M.; Singer, A.; Weyh, T. Multi-Engine in Modular Multilevel Converter based Split Battery Systems for Electric Vehicles. In Proceedings of the PCIM Europe 2019; International Exhibition and Conference for Power Electronics, Intelligent Motion, Renewable Energy, and Energy Management, Nuremberg, Germany, 7-9 May 2019; pp. 1-7. [CrossRef]

456. Theliander, O.; Kersten, A.; Kuder, M.; Han, W.; Arfa Grunditz, E.; Thiringer, T. Battery Modeling and Parameter Extraction for Drive Cycle Loss Evaluation of a Modular Battery System for Vehicles based on a Cascaded H-Bridge Multilevel Inverter. IEEE Trans. Ind. Appl. 2020, 56, 6968-6977. [CrossRef]

457. Terbrack, C.; Stottner, J.; Endisch, C. Operation of an Externally Excited Synchronous Machine with a Hybrid Multilevel Inverter. In Proceedings of the 2020 22nd European Conference on Power Electronics and Applications (EPE'20 ECCE Europe), Lyon, France, 7-11 September 2020; pp. 1-12. [CrossRef]

458. Rodriguez, J.I.; Leeb, S.B. A Multilevel Inverter Topology for Inductively Coupled Power Transfer. IEEE Trans. Power Electron. 2006, 21, 1607-1617. [CrossRef]

459. Hinago, Y.; Koizumi, H. A Single-Phase Multilevel Inverter Using Switched Series/Parallel DC Voltage Sources. IEEE Trans. Ind. Electron. 2010, 57, 2643-2650. [CrossRef]

460. Helling, F.; Gotz, S.; Weyh, T. A battery modular multilevel management system (BM3) for electric vehicles and stationary energy storage systems. In Proceedings of the 2014 16th European Conference on Power Electronics and Applications, Lappeenranta, Finland, 26-28 August 2014; pp. 1-10. [CrossRef]

461. Kim, H.; Shin, K.G. DESA: Dependable, Efficient, Scalable Architecture for Management of Large-Scale Batteries. IEEE Trans. Ind. Inform. 2012, 8, 406-417. [CrossRef]

462. Helling, F.; Kuder, M.; Singer, A.; Schmid, S.; Weyh, T. Low Voltage Power Supply in Modular Multilevel Converter based Split Battery Systems for Electrical Vehicles. In Proceedings of the 20th European Conference on Power Electronics and Applications, Riga, Latvia, 17-21 September 2018; pp. 1-10.

463. Helling, F.; Gluck, J.; Singer, A.; Weyh, T. Modular multilevel battery (M2B) for electric vehicles. In Proceedings of the 2016 18th European Conference on Power Electronics and Applications (EPE'16 ECCE Europe), Karlsruhe, Germany, 5-9 September 2016; pp. 1-9. [CrossRef]

464. Terbrack, C.; Stöttner, J.; Endisch, C. Design and Validation of the Parallel Enhanced Commutation Integrated Nested Multilevel Inverter Topology. IEEE Trans. Ind. Electron. 2021, under review. 
465. Su, G.J. Multilevel DC link inverter. In Proceedings of the Conference record of the 2004 IEEE Industry Applications Conference, Seattle, WA, USA, 3-7 October 2004; Institute of Electrical and Electronics Engineers: Piscataway, NJ, USA, 2004 ; pp. 806-812. [CrossRef]

466. Gupta, K.K.; Jain, S. Comprehensive review of a recently proposed multilevel inverter. IET Power Electron. 2014, 7, 467-479. [CrossRef]

467. Debnath, S.; Qin, J.; Bahrani, B.; Saeedifard, M.; Barbosa, P. Operation, Control, and Applications of the Modular Multilevel Converter: A Review. IEEE Trans. Power Electron. 2015, 30, 37-53. [CrossRef]

468. Babaei, E. A Cascade Multilevel Converter Topology With Reduced Number of Switches. IEEE Trans. Power Electron. 2008, 23, 2657-2664. [CrossRef]

469. Ebrahimi, J.; Babaei, E.; Gharehpetian, G.B. A New Multilevel Converter Topology With Reduced Number of Power Electronic Components. IEEE Trans. Ind. Electron. 2012, 59, 655-667. [CrossRef]

470. Buccella, C.; de Santis, C.C.E.; Khalid, H.A. Impact of the Number of Angles and Levels on Harmonic Mitigation in Multilevel Converters. In Proceedings of the IECON 2015, Yokohama, Japan, 9-12 November 2015; Ohishi, K., Hashimoto, H., Eds.; IEEE: Piscataway, NJ, USA, 2015; pp. 002834-002839. [CrossRef]

471. Gupta, K.K.; Jain, S. Topology for multilevel inverters to attain maximum number of levels from given DC sources. IET Power Electron. 2012, 5, 435. [CrossRef]

472. Singer, A.; Helling, F.; Weyh, T.; Jungbauer, J.; Pfisterer, H.J. Modular multilevel parallel converter based split battery system (M2B) for stationary storage applications. In Proceedings of the 2017 19th European Conference on Power Electronics and Applications (EPE'17 ECCE Europe), Warsaw, Poland, 11-14 September 2017; pp. P.1-P.10. [CrossRef]

473. Rech, C.; Pinheiro, J.R. Hybrid Multilevel Converters: Unified Analysis and Design Considerations. IEEE Trans. Ind. Electron. 2007, 54, 1092-1104. [CrossRef]

474. Helling, F.; Gotz, S.; Singer, A.; Weyh, T. Fast Modular Multilevel series/Parallel Converter for direct-drive gas turbines. In Proceedings of the 2015 IEEE North West Russia Section Young Researchers in Electrical and Electronic Engineering Conference (2015 ElConRusNW), St. Petersburg, Russia, 2-4 February 2015; Shaposhnikov, S.O., Ed.; IEEE: Piscataway, NJ, USA, 2015; pp. 198-202. [CrossRef]

475. Liang, H.; Guo, L.; Song, J.; Yang, Y.; Zhang, W.; Qi, H. State-of-Charge Balancing Control of a Modular Multilevel Converter with an Integrated Battery Energy Storage. Energies 2018, 11, 873. [CrossRef]

476. Marquardt, R. Modular Multilevel Converter: An universal concept for HVDC-Networks and extended DC-Bus-applications. In Proceedings of the International Power Electronics Conference (IPEC), Sapporo, Japan, 21-24 June 2010; IEEE: Piscataway, NJ, USA, 2010; pp. 502-507. [CrossRef]

477. Chang, F.; Ilina, O.; Lienkamp, M.; Voss, L. Improving the Overall Efficiency of Automotive Inverters Using a Multilevel Converter Composed of Low Voltage Si mosfets. IEEE Trans. Power Electron. 2019, 34, 3586-3602. [CrossRef]

478. Rodriguez, J.; Lai, J.S.; Peng, F.Z. Multilevel inverters: A survey of topologies, controls, and applications. IEEE Trans. Ind. Electron. 2002, 49, 724-738. [CrossRef]

479. Lai, J.S.; Peng, F.Z. Multilevel converters-a new breed of power converters. Electron. Lett. 1996, 32, 509-517. [CrossRef]

480. El-Hosainy, A.; Hamed, H.A.; Azazi, H.Z.; El-Kholy, E.E. A review of multilevel inverter topologies, control techniques, and applications. In Proceedings of the 2017 Nineteenth International Middle East Power Systems Conference (MEPCON), Cairo, Egypt, 19-21 December 2017; pp. 1265-1275. [CrossRef]

481. Rodriguez, J.; Franquelo, L.G.; Kouro, S.; Leon, J.I.; Portillo, R.C.; Prats, M.; Perez, M.A. Multilevel Converters: An Enabling Technology for High-Power Applications. Proc. IEEE 2009, 97, 1786-1817. [CrossRef]

482. Deng, Y.; Harley, R.G. Space-Vector Versus Nearest-Level Pulse Width Modulation for Multilevel Converters. IEEE Trans. Power Electron. 2015, 30, 2962-2974. [CrossRef]

483. Swamy, D.M.; Venkatesan, M. Three Phase Multi-Level Inverter Topologies and Modulation Techniques: A Short review. In Proceedings of the 2019 2nd International Conference on Intelligent Computing, Instrumentation and Control Technologies (ICICICT), Kannur, India, 5-6 July 2019; pp. 305-311. [CrossRef]

484. Dupont, F.H.; Pinheiro, J.R.; Montagner, V.F.; Pinheiro, H. GA optimization to mitigate voltage harmonics of cascade multilevel converters. In Proceedings of the IEEE Chilecon2015, Santiago, Chile, 28-30 October 2015; Fernández-Fernández, M., Ed.; IEEE: Piscataway, NJ, USA, 2015; pp. 461-466. [CrossRef]

485. Li, L.; Czarkowski, D.; Liu, Y.; Pillay, P. Multilevel selective harmonic elimination PWM technique in series-connected voltage inverters. IEEE Trans. Ind. Appl. 2000, 36, 160-170. [CrossRef]

486. Rodriguez, J.; Moran, L.; Correa, P.; Silva, C. A vector control technique for medium-voltage multilevel inverters. IEEE Trans. Ind. Electron. 2002, 49, 882-888. [CrossRef]

487. Attique, Q.M. A Survey on Space-Vector Pulse Width Modulation for Multilevel Inverters. CPSS Trans. Power Electron. Appl. 2017, 2, 226-236. [CrossRef]

488. Prasad, K.N.V.; ParimitaPradhan, P.; Misra, B.; Surekha, J. A modified space vector algorithm for 5-level cascaded multilevel inverter. In Proceedings of the 2017 Innovations in Power and Advanced Computing Technologies (i-PACT), Vellore, India, 21-22 April 2017; pp. 1-6. [CrossRef]

489. Franquelo, L.G.; León, J.I.; Prats, M.M.; Portillo, R. Space Vector Modulation techniques for multilevel converters-A survey. Prz. Elektrotech. 2006, 82, 56-61. 
490. Mostafa, M.; Abdou, A.F.; El-Gawad, A.F.A.; El-Kholy, E.E. Comparison of multi-carrier and SHE-PWM for a nine levels cascaded H-bridge inverter. In Proceedings of the 2017 Nineteenth International Middle East Power Systems Conference (MEPCON), Cairo, Egypt, 19-21 December 2017; pp. 1483-1491. [CrossRef]

491. Becker, J.; Nemeth, T.; Wegmann, R.; Sauer, D. Dimensioning and Optimization of Hybrid Li-Ion Battery Systems for EVs. World Electr. Veh. J. 2018, 9, 19. [CrossRef]

492. Tu, C.H.; Emadi, A. A novel series-parallel reconfigurable hybrid energy storage system for electrified vehicles. In Proceedings of the 2012 IEEE Transportation Electrification Conference and Expo (ITEC), Dearborn, MI, USA, 18-20 June 2012; pp. 1-4. [CrossRef]

493. Ciccarelli, F.; Clemente, G.; Iannuzzi, D. Energy storage management control based on supercapacitors using a modular multilevel inverter topology for electrical vehicles. In Proceedings of the 2013 International Conference on Clean Electrical Power (ICCEP), Alghero, Italy, 11-13 June 2013; pp. 170-176. [CrossRef]

494. Guo, F.; Sharma, R. A modular multilevel converter with half-bridge submodules for hybrid energy storage systems integrating battery and UltraCapacitor. In Proceedings of the 2015 IEEE Applied Power Electronics Conference and Exposition (APEC), Charlotte, NC, USA, 15-19 March 2015; pp. 3025-3030. [CrossRef]

495. Bharadwaj, C.A.; Maiti, S. Modular multilevel converter based hybrid energy storage system. In Proceedings of the IEEE PES Asia-Pacific Power and Energy Engineering Conference 2017, Bangalore, India, 8-10 November 2017; pp. 1-6. [CrossRef]

496. George, S.S.; Badawy, M.O. A Modular Multi-Level Converter for Energy Management of Hybrid Storage System in Electric Vehicles. In Proceedings of the 2018 IEEE Transportation Electrification Conference and Expo (ITEC), Long Beach, CA, USA, 13-15 June 2018; pp. 336-341. [CrossRef]

497. Zimmermann, T.; Mora, M.; Steinhorst, S.; Mueller-Gritschneder, D.; Jossen, A. Analysis of Dissipative Losses in Modular Reconfigurable Energy Storage Systems Using SystemC TLM and SystemC-AMS. ACM Trans. Des. Autom. Electron. Syst. 2019, 24, 1-33. [CrossRef]

498. Zimmermann, T.; Keil, P.; Hofmann, M.; Horsche, M.F.; Pichlmaier, S.; Jossen, A. Review of system topologies for hybrid electrical energy storage systems. J. Energy Storage 2016, 8, 78-90. [CrossRef]

499. Praveena Krishna, P.S.; Sabhahit, J.N.; Kedlaya, A. Energy Management Strategies for Hybrid Energy Storage System in Electric Vehicles: A Review. In Proceedings of the 2020 IEEE International Conference on Electronics, Computing and Communication Technologies (CONECCT), Bangalore, India, 2-4 July 2020; pp. 1-7. [CrossRef]

500. Jiang, Z.; Gao, L.; Dougal, R.A. Adaptive Control Strategy for Active Power Sharing in Hybrid Fuel Cell/Battery Power Sources. IEEE Trans. Energy Convers. 2007, 22, 507-515. [CrossRef]

501. Song, Z.; Hofmann, H.; Li, J.; Hou, J.; Han, X.; Ouyang, M. Energy management strategies comparison for electric vehicles with hybrid energy storage system. Appl. Energy 2014, 134, 321-331. [CrossRef]

502. Santucci, A.; Sorniotti, A.; Lekakou, C. Power split strategies for hybrid energy storage systems for vehicular applications. J. Power Sources 2014, 258, 395-407. [CrossRef]

503. Zhou, W.; Li, M.; Yin, H.; Ma, C. An adaptive fuzzy logic based energy management strategy for electric vehicles. In Proceedings of the 2014 IEEE 23rd International Symposium on Industrial Electronics (ISIE), Istanbul, Turkey, 1-4 June 2014; pp. 1778-1783. [CrossRef]

504. Zhang, Q.; Deng, W. An Adaptive Energy Management System for Electric Vehicles Based on Driving Cycle Identification and Wavelet Transform. Energies 2016, 9, 341. [CrossRef]

505. Hu, J.; Jiang, X.; Jia, M.; Zheng, Y. Energy Management Strategy for the Hybrid Energy Storage System of Pure Electric Vehicle Considering Traffic Information. Appl. Sci. 2018, 8, 1266. [CrossRef]

506. Shen, J.; Khaligh, A. A Supervisory Energy Management Control Strategy in a Battery/Ultracapacitor Hybrid Energy Storage System. IEEE Trans. Transp. Electrif. 2015, 1, 223-231. [CrossRef]

507. Chenghui, Z.; Qingsheng, S.; Naxin, C.; Wuhua, L. Particle Swarm Optimization for energy management fuzzy controller design in dual-source electric vehicle. In Proceedings of the IEEE Power Electronics Specialists Conference 2007, Orlando, FL, USA, 17-21 June 2007; pp. 1405-1410. [CrossRef]

508. Mesbahi, T.; Rizoug, N.; Bartholomeus, P.; Sadoun, R.; Khenfri, F.; Lemoigne, P. Optimal Energy Management for a Li-Ion Battery/Supercapacitor Hybrid Energy Storage System Based on Particle Swarm Optimization Incorporating Nelder-Mead Simplex Approach. IEEE Trans. Intell. Veh. 2017, 2, 99-110. [CrossRef]

509. Awerbuch, J.J.; Sullivan, C.R. Filter-based power splitting in ultracapacitor-battery hybrids for vehicular applications. In Proceedings of the 2010 IEEE 12th Workshop on Control and Modeling for Power Electronics (COMPEL), Boulder, CO, USA, 28-30 June 2010; pp. 1-8. [CrossRef]

510. Wang, B.; Wang, C.; Ma, G.; Zhang, L. Power-split strategy based on average power method for semi-active hybrid energy storage system in small electric vehicles. Energy Procedia 2019, 158, 2994-2999. [CrossRef]

511. Xie, Q.; Lin, X.; Wang, Y.; Pedram, M.; Shin, D.; Chang, N. State of health aware charge management in hybrid electrical energy storage systems. In Proceedings of the 2012 Design, Automation \& Test in Europe Conference \& Exhibition (DATE), Dresden, Germany, 12-16 March 2012; pp. 1060-1065. [CrossRef]

512. Zhang, X.; Mi, C.C.; Masrur, A.; Daniszewski, D. Wavelet-transform-based power management of hybrid vehicles with multiple on-board energy sources including fuel cell, battery and ultracapacitor. J. Power Sources 2008, 185, 1533-1543. [CrossRef] 
513. Hu, J.; Liu, D.; Du, C.; Yan, F.; Lv, C. Intelligent energy management strategy of hybrid energy storage system for electric vehicle based on driving pattern recognition. Energy 2020, 198, 117298. [CrossRef]

514. Romaus, C.; Gathmann, K.; Bocker, J. Optimal energy management for a hybrid energy storage system for electric vehicles based on Stochastic Dynamic Programming. In Proceedings of the 2010 IEEE Vehicle Power and Propulsion Conference, Lille, France, 1-3 September 2010; pp. 1-6. [CrossRef]

515. Wegmann, R.; Döge, V.; Becker, J.; Sauer, D.U. Optimized operation of hybrid battery systems for electric vehicles using deterministic and stochastic dynamic programming. J. Energy Storage 2017, 14, 22-38. [CrossRef]

516. Zhou, F.; Xiao, F.; Chang, C.; Shao, Y.; Song, C. Adaptive Model Predictive Control-Based Energy Management for Semi-Active Hybrid Energy Storage Systems on Electric Vehicles. Energies 2017, 10, 1063. [CrossRef]

517. Trovão, J.P.; Pereirinha, P.G.; Jorge, H.M.; Antunes, C.H. A multi-level energy management system for multi-source electric vehicles-An integrated rule-based meta-heuristic approach. Appl. Energy 2013, 105, 304-318. [CrossRef]

518. Trovao, J.P.F.; Santos, V.D.N.; Antunes, C.H.; Pereirinha, P.G.; Jorge, H.M. A Real-Time Energy Management Architecture for Multisource Electric Vehicles. IEEE Trans. Ind. Electron. 2015, 62, 3223-3233. [CrossRef]

519. Choi, M.E.; Lee, J.S.; Seo, S.W. Real-Time Optimization for Power Management Systems of a Battery/Supercapacitor Hybrid Energy Storage System in Electric Vehicles. IEEE Trans. Veh. Technol. 2014, 63, 3600-3611. [CrossRef]

520. Yue, S.; Wang, Y.; Xie, Q.; Zhu, D.; Pedram, M.; Chang, N. Model-free learning-based online management of hybrid electrical energy storage systems in electric vehicles. In Proceedings of the IECON 2014-40th Annual Conference of the IEEE Industrial Electronics Society, Dallas, TX, USA, 29 October-1 November 2014; pp. 3142-3148. [CrossRef]

521. Cao, J.; Xiong, R. Reinforcement Learning-based Real-time Energy Management for Plug-in Hybrid Electric Vehicle with Hybrid Energy Storage System. Energy Procedia 2017, 2017, 1896-1901. [CrossRef]

522. Zhang, S.; Xiong, R.; Cao, J. Battery durability and longevity based power management for plug-in hybrid electric vehicle with hybrid energy storage system. Appl. Energy 2016, 179, 316-328. [CrossRef]

523. Li, W.; Cui, H.; Nemeth, T.; Jansen, J.; Ünlübayir, C.; Wei, Z.; Zhang, L.; Wang, Z.; Ruan, J.; Dai, H.; et al. Deep reinforcement learning-based energy management of hybrid battery systems in electric vehicles. J. Energy Storage 2021, 36, 102355. [CrossRef]

524. Yu, H.; Cao, D. Multi-objective Optimal Sizing and Real-time Control of Hybrid Energy Storage Systems for Electric Vehicles. In Proceedings of the IEEE Intelligent Vehicles Symposium 2018, Changshu, China, 26-30 June 2018; pp. 191-196. [CrossRef]

525. Yu, H.; Castelli-Dezza, F.; Cheli, F.; Tang, X.; Hu, X.; Lin, X. Dimensioning and Power Management of Hybrid Energy Storage Systems for Electric Vehicles With Multiple Optimization Criteria. IEEE Trans. Power Electron. 2021, 36, 5545-5556. [CrossRef]

526. Hung, Y.H.; Wu, C.H. An integrated optimization approach for a hybrid energy system in electric vehicles. Appl. Energy 2012, 98, 479-490. [CrossRef]

527. Baronti, F.; Fantechi, G.; Roncella, R.; Saletti, R. Intelligent Cell Gauge for a Hierarchical Battery Management System. In Proceedings of the IEEE Transactions on Transportation Electrification, Dearborn, MI, USA, 18-20 June 2012. [CrossRef]

528. Rößler, W. When Do We Get the Electronic Battery Switch? In Advanced Microsystems for Automotive Applications 2014; FischerWolfarth, J., Meyer, G., Eds.; Lecture Notes in Mobility; Springer International Publishing: Cham, Switzerland, $2014 ;$ pp. 165-177. [CrossRef]

529. Kim, T.; Qiao, W.; Qu, L. Series-connected self-reconfigurable multicell battery. In Proceedings of the 2011 Twenty-Sixth Annual IEEE Applied Power Electronics Conference and Exposition (APEC), Fort Worth, TX, USA, 6-11 March 2011; pp. 1382-1387. [CrossRef]

530. Steinhorst, S.; Shao, Z.; Chakraborty, S.; Kauer, M.; Li, S.; Lukasiewycz, M.; Narayanaswamy, S.; Rafique, M.U.; Wang, Q. Distributed reconfigurable Battery System Management Architectures. In Proceedings of the 2016 21st Asia and South Pacific Design Automation Conference (ASP-DAC), Macao, China, 25-28 January 2016; pp. 429-434. [CrossRef]

531. Hanzl, C.; Hartmann, C.; Hölzle, M.; Liebhart, B.; Schmid, M.; Endisch, C. Current Commutation in a Switched Lithium-Ion Cell used in Cascaded Half-Bridge Multilevel Inverters. IET Power Electron. 2021, 14, 1073-1088. [CrossRef]

532. Shalchi Alishah, R.; Nazarpour, D.; Hosseini, S.H.; Sabahi, M. Novel multilevel inverter topologies for medium and high-voltage applications with lower values of blocked voltage by switches. IET Power Electron. 2014, 7, 3062-3071. [CrossRef]

533. Ng, K.K. Complete Guide to Semiconductor Devices; John Wiley \& Sons, Inc.: Hoboken, NJ, USA, 2009. [CrossRef]

534. Ci, S.; Zhang, J.; Sharif, H.; Alahmad, M. (Eds.) Dynamic Reconfigurable Multi-Cell Battery: A Novel Approach to Improve Battery Performance. In Proceedings of the Twenty-Seventh Annual IEEE Applied Power Electronics Conference and Exposition (APEC), Orlando, FL, USA, 5-9 February 2012; IEEE: Piscataway, NJ, USA, 2012; pp. 439-442 [CrossRef]

535. Ji, F.; Liao, L.; Wu, T.; Chang, C.; Wang, M. Self-reconfiguration batteries with stable voltage during the full cycle without the DC-DC converter. J. Energy Storage 2020, 28, 101213. [CrossRef]

536. Lutz, J.; Schlangenotto, H.; Scheuermann, U.; de Doncker, R. Semiconductor Power Devices; Springer International Publishing: Cham, Switzerland, 2018. [CrossRef]

537. Haghdar, K. Optimal DC Source Influence on Selective Harmonic Elimination in Multilevel Inverters Using Teaching-LearningBased Optimization. IEEE Trans. Ind. Electron. 2020, 67, 942-949. [CrossRef]

538. Haritha, M.; Sujith, S. DC link voltage balancing of three phase neutral point clamped multilevel inverter by redundant vector identification. In Proceedings of the IEEE International Conference on Power, Control, Signals and Instrumentation Engineering 2017, Chennai, India, 21-22 September 2017; pp. 1952-1958. [CrossRef] 
539. Ma, Y.; Lin, H.; Wang, Z.; Ze, Z. Modified State-of-Charge Balancing Control of Modular Multilevel Converter with Integrated Battery Energy Storage System. Energies 2019, 12, 96. [CrossRef]

540. Memon, M.A.; Mekhilef, S.; Mubin, M. Selective harmonic elimination in multilevel inverter using hybrid APSO algorithm. IET Power Electron. 2018, 11, 1673-1680. [CrossRef]

541. Tolbert, L.M.; Peng, F.Z.; Habetler, T.G. Multilevel inverters for electric vehicle applications. In Proceedings of the Power Electronics in Transportation (Cat. No.98TH8349), Dearborn, MI, USA, 22-23 October 1998; pp. 79-84. [CrossRef]

542. Baliga, B.J. Fundamentals of Power Semiconductor Devices; Springer International Publishing: Cham, Switzerland, 2019. [CrossRef]

543. Engelhardt, J.; Gabderakhmanova, T.; Rohde, G.; Marinelli, M. Reconfigurable Stationary Battery with Adaptive Cell Switching for Electric Vehicle Fast-Charging. In Proceedings of the 2020 55th International Universities Power Engineering Conference (UPEC), Turin, Italy, 1-4 September 2020; pp. 1-6. [CrossRef]

544. Gao, Z.; Lu, Q. A Hybrid Cascaded Multilevel Converter Based on Three-Level Cells for Battery Energy Management Applied in Electric Vehicles. IEEE Trans. Power Electron. 2019, 34, 7326-7349. [CrossRef]

545. Lee, K.M.; Kim, D.H.; Shin, J.H.; Goh, T.D.; Park, J.H.; Ryu, Y.H. Reconfigurable battery system with active balancing circuit. In Proceedings of the IECON 2019-45th Annual Conference of the IEEE Industrial Electronics Society, Lisbon, Portugal, 14-17 October 2019; pp. 4583-4586. [CrossRef]

546. Patel, V.; Tinari, M.; Buccella, C.; Cecati, C. Analysis on Multilevel Inverter Powertrains for E-transportation. In Proceedings of the 2019 IEEE 13th International Conference on Compatibility, Power Electronics and Power Engineering (CPE-POWERENG), Sonderborg, Denmark, 23-25 April 2019; pp. 1-6. [CrossRef]

547. Sathik, M.J.; Bhatnagar, K.; Siwakoti, Y.P.; Bassi, H.M.; Rawa, M.; Sandeep, N.; Yang, Y.; Blaabjerg, F. Switched-capacitor multilevel inverter with self-voltage-balancing for high-frequency power distribution system. IET Power Electron. 2020, 13, 1807-1818. [CrossRef]

548. Schettino, G.; Viola, F.; Di Tommaso, A.O.; Livreri, P.; Miceli, R. Experimental Validation of a Novel Method for Harmonic Mitigation for a Three-Phase Five-Level Cascaded H-Bridges Inverter. IEEE Trans. Ind. Appl. 2019, 55, 6089-6101. [CrossRef]

549. Sheir, A.; Youssef, M.Z. A Novel Power Balancing Technique in Neutral Point Clamping Multilevel Inverters for the Electric Vehicle Industry under Distributed Unbalance Battery Powering Scheme. In Proceedings of the 2019 IEEE Applied Power Electronics Conference and Exposition (APEC), Anaheim, CA, USA, 17-21 March 2019; pp. 3304-3308. [CrossRef]

550. Sheir, A.; Youssef, M.Z.; Orabi, M. A Novel Bidirectional T-Type Multilevel Inverter for Electric Vehicle Applications. IEEE Trans. Power Electron. 2019, 34, 6648-6658. [CrossRef]

551. Sheir, A.; Youssef, M.Z.; Orabi, M. A Novel Auxiliary Modular Inverter with Battery Integration for Electric Vehicle Applications. In Proceedings of the 2019 IEEE Applied Power Electronics Conference and Exposition (APEC), Anaheim, CA, USA, 17-21 March 2019; pp. 1730-1737. [CrossRef]

552. Drivetek AG. Electric, Drive, Technology, Products, Multilevel, Highspeed, Inverter. 2019. Available online: https://www. drivetek.ch (accessed on 8 September 2021). [CrossRef]

553. Reindl, A.; Meier, H.; Niemetz, M. Scalable, Decentralized Battery Management System Based on Self-organizing Nodes. In Proceedings of the International Conference on Architecture of Computing Systems, Aachen, Germany, 25-28 May 2020; pp. 171-184.

554. Brandl, M.; Gall, H.; Wenger, M.; Lorentz, V.; Giegerich, M.; Baronti, F.; Fantechi, G.; Fanucci, L.; Roncella, R.; Saletti, R.; et al. Batteries and battery management systems for electric vehicles. In Proceedings of the 2012 Design, Automation \& Test in Europe Conference \& Exhibition (DATE), Dresden, Germany, 12-16 March 2012; pp. 971-976. [CrossRef]

555. Otto, A.; Rzepka, S.; Mager, T.; Michel, B.; Lanciotti, C.; Günther, T.; Kanoun, O. Battery Management Network for Fully Electrical Vehicles Featuring Smart Systems at Cell and Pack Level. In Advanced Microsystems for Automotive Applications 2012; Meyer, G., Ed.; Springer: Berlin/Heidelberg, Germany, 2012. [CrossRef]

556. Baronti, F.; Fantechi, G.; Leonardi, E.; Roncella, R.; Saletti, R. Hierarchical platform for monitoring, managing and charge balancing of LiPo batteries. In Proceedings of the 2011 IEEE Vehicle Power and Propulsion Conference, Chicago, IL, USA, 6-9 September 2011; pp. 1-6. [CrossRef]

557. Linlin, L.; Xu, Z.; Zhujinsheng.; Jing, X.; Shuntao, X. Research on dynamic equalization for lithium battery management system. In Proceedings of the 2017 29th Chinese Control in Addition, Decision Conference (CCDC), Chongqing, China, 28-30 May 2017; pp. 6884-6888. [CrossRef]

558. Narayanaswamy, S.; Park, S.; Steinhorst, S.; Chakraborty, S. Design automation for battery systems. In Proceedings of the International Conference on Computer-Aided Design-ICCAD '18, San Diego, CA, USA, 5-8 November 2018; Bahar, I., Ed.; ACM Press: New York, NY, USA, 2018; pp. 1-7. [CrossRef]

559. Rahimi-Eichi, H.; Ojha, U.; Baronti, F.; Chow, M.Y. Battery Management System: An Overview of Its Application in the Smart Grid and Electric Vehicles. IEEE Ind. Electron. Mag. 2013, 7, 4-16. [CrossRef]

560. Steinhorst, S.; Lukasiewycz, M.; Narayanaswamy, S.; Kauer, M.; Chakraborty, S. Smart Cells for Embedded Battery Management. In Proceedings of the 2014 IEEE International Conference on Cyber-Physical Systems, Networks, and Applications, Hong Kong, China, 25-26 August 2014; pp. 59-64. [CrossRef]

561. Li, Y.; Han, Y. A Module-Integrated Distributed Battery Energy Storage and Management System. IEEE Trans. Power Electron. 2016, 31, 8260-8270. [CrossRef] 
562. Kim, C.H.; Kim, M.Y.; Moon, G.W. A Modularized Charge Equalizer Using a Battery Monitoring IC for Series-Connected Li-Ion Battery Strings in Electric Vehicles. IEEE Trans. Power Electron. 2013, 28, 3779-3787. [CrossRef]

563. Wenger, M.M.; Filimon, R.; Lorentz, V.; Marz, M. A robust contactless capacitive communication link for high power battery systems. In Proceedings of the 2014 IEEE 23rd International Symposium on Industrial Electronics (ISIE), Istanbul, Turkey, 1-4 June 2014; pp. 1766-1772. [CrossRef]

564. Lorentz, V.; Wenger, M.M.; Grosch, J.L.; Giegerich, M.; Jank, M.; Marz, M.; Frey, L. Novel cost-efficient contactless distributed monitoring concept for smart battery cells. In Proceedings of the 2012 IEEE International Symposium on Industrial Electronics, Hangzhou, China, 28-31 May 2012; pp. 1342-1347. [CrossRef]

565. Zhang, R.; Wu, J.; Wang, R.; Yan, R.; Zhu, Y.; He, X. A Novel Battery Management System Architecture Based on an Isolated Power/Data Multiplexing Transmission Bus. IEEE Trans. Ind. Electron. 2019, 66, 5979-5991. [CrossRef]

566. Schneider, M.; Ilgin, S.; Jegenhorst, N.; Kube, R.; Puttjer, S.; Riemschneider, K.R.; Vollmer, J. Automotive battery monitoring by wireless cell sensors. In Proceedings of the 2012 IEEE International Instrumentation and Measurement Technology Conference Proceedings, Graz, Austria, 13-16 May 2012; pp. 816-820. [CrossRef]

567. Li, Y.; Han, Y. Power electronics integration on battery cells. In Proceedings of the 2014 IEEE Applied Power Electronics Conference and Exposition-APEC 2014, Fort Worth, TX, USA, 16-20 March 2014; pp. 3318-3322. [CrossRef]

568. Steinhorst, S. Design and verification methodologies for Smart Battery Cells. In Proceedings of the 2016 International Symposium on Integrated Circuits (ISIC), Singapore, 12-14 December 2016; pp. 1-4. [CrossRef]

569. Chowdhury, S.M.; Badawy, M.O.; Sozer, Y.; Garcia, J.A.D.A. A Novel Battery Management System Using the Duality of the Adaptive Droop Control Theory. IEEE Trans. Ind. Appl. 2019, 55, 5078-5088. [CrossRef]

570. Frost, D.F.; Howey, D.A. Completely Decentralized Active Balancing Battery Management System. IEEE Trans. Power Electron. 2018, 33, 729-738. [CrossRef]

571. Lafrenz, J.L.; Scheff, P.; Ricco, M.; Kerekes, T.; Olsen, R.L.; Teodorescu, R.; Liserre, M. Application Layer Design for Smart Battery Pack Control with Wi-Fi ${ }^{\circledR}$ Feedback. In Proceedings of the 2018 IEEE Energy Conversion Congress and Exposition (ECCE), Portland, OR, USA, 23-27 September 2018; pp. 5586-5590. [CrossRef]

572. Vogt, T. Comparing wired vs. wireless solutions in automotive battery management systems. In Automotive TI Tech Days 2020, Online, 22-24 September 2020; Texas Instruments: Dallas, TX, USA, 2020.

573. ISO. ISO 26262-(1-12):2018 Road Vehicles—Functional Safety; International Organization for Standardization: Geneva, Switzerland, 2018.

574. Tabatowski-Bush, B. Functional Safety for Battery Monitoring Integrated Circuits. In Proceedings of the WCX ${ }^{\mathrm{TM}}$ 17: SAE World Congress Experience, Detroit, MI, USA, 4-6 April 2017. [CrossRef]

575. Lee, M.; Lee, J.; Lee, I.; Lee, J.; Chon, A. Wireless battery management system. In Proceedings of the World Electric Vehicle Symposium and Exposition (EVS 27), Barcelona, Spain, 17-20 November 2013; IEEE: Piscataway, NJ, USA, 2013; pp. 1-5. [CrossRef]

576. Huang, X.; Sui, X.; Stroe, D.I.; Teodorescu, R. A Review of Management Architectures and Balancing Strategies in Smart Batteries. In Proceedings of the IECON 2019—45th Annual Conference of the IEEE Industrial Electronics Society, Lisbon, Portugal, 14-17 October 2019; pp. 5909-5914. [CrossRef]

577. Alonso, D.; Opalko, O.; Dostert, K. Channel Measurements and Simulations with Planar Inverted F-Antennas in an Enhanced Testbed for a Wireless Battery Management System. In Proceedings of the WSA 2015, 19th International ITG Workshop on Smart Antennas, Ilmenau, Germany, 3-5 March 2015; pp. 1-8.

578. Texas Instruments. BQ7961x-Q1 Family of 12S, 14S, $16 S$ Precision Automotive Battery Monitor, Balancer and Integrated Hardware Protector; Texas Instruments: Dallas, TX, USA, 2020.

579. Infineon. TLE9015QU BMS Transceiver IC-UART to iso UART; Infineon: Munich, Germany, 2019.

580. NXP Semiconductors. MC33771B_SDS Battery Cell Controller IC; Rev. 6.0 Product Short Data Sheet; NXP Semiconductors: Eindhoven, The Netherlands, 2020.

581. Kilic, A.; Koroglu, S.; Demirçalı, A.; Kesler, S.; Oner, Y.; Karakas, E.; Sergeant, P. Design of Master and Slave Modules on Battery Management System for Electric Vehicles. In Proceedings of the (2017) 6th International Conference on Advanced Technology \& Sciences (ICAT’Riga), Riga, Latvia, 12-15 September 2017; pp. 161-166.

582. Toh, C.L.; Norum, L.E. A high speed control network synchronization jitter evaluation for embedded monitoring and control in modular multilevel converter. In Proceedings of the 2013 IEEE Grenoble Conference, Grenoble, France, 16-20 June 2013; pp. 1-6. [CrossRef]

583. Dan Burlacu, P.; Mathe, L.; Teodorescu, R. Synchronization of the distributed PWM carrier waves for modular multilevel converters. In Proceedings of the 2014 International Conference on Optimization of Electrical and Electronic Equipment (OPTIM), Bran, Romania, 22-24 May 2014; pp. 553-559. [CrossRef]

584. IEEE. IEEE Standard for Ethernet Amendment 1: Physical Layer Specifications and Management Parameters for 100 Mbs Operation over a Single Balanced Twisted Pair Cable (100BASE-T1); IEEE: Piscataway, NJ, USA, 2015.

585. Bae, J.H.; Zhiguo, B.; Koo, B.J.; Kim, H.R.; Jang, D.G. The CAN communication application on the BMS. In Proceedings of the 2013 International Conference on ICT Convergence (ICTC), Jeju, Korea, 14-16 October 2013; pp. 1140-1145. [CrossRef] 
586. Reindl, A.; Meier, H.; Niemetz, M. Software Framework for the Simulation of a Decentralized Battery Management System Consisting of Intelligent Battery Cells. In Proceedings of the 2019 IEEE Student Conference on Research and Development (SCOReD), Bandar Seri Iskandar, Malaysia, 15-17 October 2019; pp. 75-80. [CrossRef]

587. Bilbao, J.; Varela, C.; Bravo, E.; Rodríguez, M.; García, O.; González, P. Alternatives for a design of a Battery Management System for traction applications. In Proceedings of the 2013 12th WSEAS International Conference on Applications of Computer Engineering (ACE 2013), Cambridge, MA, USA, 1-30 January 2013.

588. Alonso, D.; Opalko, O.; Sigle, M.; Dostert, K. Towards a Wireless Battery Management System: Evaluation of Antennas and Radio Channel Measurements Inside a Battery Emulator. In Proceedings of the 2014 IEEE 80th Vehicular Technology Conference (VTC2014-Fall), Vancouver, BC, Canada, 14-17 September 2014; pp. 1-5. [CrossRef]

589. Robert Bosch GmbH. CAN XL: Next, Step in CAN Evolution; Robert Bosch GmbH: Gerlingen, Germany, 2020.

590. Morello, R.; Schwarz, R.; Hoedemaekers, E.R.G.; Habenschaden, F.; Di Rienzo, R.; Roncella, R.; Saletti, R.; Rosca, B.; Steffenhagen, T.; Lorentz, V.R.H.; et al. Implementation and Test of a 48 V Smart Battery System with Integrated DC/DC Converter. In Proceedings of the 2019 IEEE 28th International Symposium on Industrial Electronics (ISIE), Vancouver, BC, Canada, 12-14 June 2019. [CrossRef]

591. Saleem, M.S. Development of PLC based Communication Architecture for Battery Management System. In Proceedings of the 2020 IEEE 91st Vehicular Technology Conference (VTC2020-Spring), Antwerp, Belgium, 25-28 May 2020; pp. 1-5. [CrossRef]

592. Saidani, F.; Hutter, F.X.; Scurtu, R.G.; Braunwarth, W.; Burghartz, J.N. Lithium-ion battery models: A comparative study and a model-based powerline communication. Adv. Radio Sci. 2017, 15, 83-91. [CrossRef]

593. Opalko, O.; Alonso, D.; Dostert, K. Measurements on Rogowski coils as coupling elements for power line communication in traction lithium-ion batteries. In Proceedings of the 2015 IEEE International Symposium on Power Line Communications and Its Applications (ISPLC), Austin, TX, USA, 29 March-1 April 2015; pp. 29-34. [CrossRef]

594. Ouannes, I.; Nickel, P.; Bernius, J.; Dostert, K. Physical Layer Performance Analysis of Power Line Communication (PLC) Applied for Cell-Wise Monitoring of Automotive Lithium-Ion Batteries. In Proceedings of the OFDM 2014; 18th International OFDM Workshop 2014 (InOWo'14), Essen, Germany, 27-28 August 2014; pp. 1-8.

595. Ouannes, I.; Nickel, P.; Dostert, K. Cell-wise monitoring of Lithium-ion batteries for automotive traction applications by using power line communication: Battery modeling and channel characterization. In Proceedings of the 18th IEEE International Symposium on Power Line Communications and Its Applications, Glasgow, UK, 30 March-2 April 2014; pp. $24-29$.

596. Lara, D.; Porter, D. Using Wireless Technologies to Replace Cables in Car Access and Battery Management Systems in Automotive; Texas Instruments: Dallas, TX, USA, 2020.

597. Kumbhar, S.; Faika, T.; Makwana, D.; Kim, T.; Lee, Y. Cybersecurity for Battery Management Systems in Cyber-Physical Environments. In Proceedings of the 2018 IEEE Transportation Electrification Conference and Expo (ITEC), Long Beach, CA, USA, 13-15 June 2018; pp. 934-938. [CrossRef]

598. Alonso, D.; Opalko, O.; Dostert, K. Parametrization of automotive lithium-ion batteries and its influence on the wireless in-battery channel. In Proceedings of the 2016 10th European Conference on Antennas and Propagation (EuCAP), Davos, Switzerland, 10-15 April 2016; pp. 1-5. [CrossRef]

599. Mathew, S.A.; Prakash, R.; John, P.C. A smart wireless battery monitoring system for Electric Vehicles. In Proceedings of the 2012 12th International Conference on Intelligent Systems Design and Applications (ISDA), Kochi, India, 27-29 November 2012; pp. 189-193. [CrossRef]

600. Wu, Y.; Liao, X.; Chen, W.; Chen, D. A Battery Management System for electric vehicle based on Zigbee and CAN. In Proceedings of the 2011 4th International Congress on Image and Signal Processing, Shanghai, China, 15-17 October 2011; pp. $2517-2521$. [CrossRef]

601. Shell, C.; Henderson, J.; Verra, H.; Dyer, J. Implementation of a wireless battery management system (WBMS). In Proceedings of the 2015 IEEE International Instrumentation and Measurement Technology Conference (I2MTC) Proceedings, Pisa, Italy, 11-14 May 2015; pp. 1954-1959. [CrossRef]

602. Huang, X.; Acharya, A.B.; Meng, J.; Sui, X.; Stroe, D.I.; Teodorescu, R. Wireless Smart Battery Management System for Electric Vehicles. In Proceedings of the 2020 IEEE Energy Conversion Congress and Exposition (ECCE), Detroit, MI, USA, 11-15 October 2020; pp. 5620-5625. [CrossRef]

603. Kumtachi, T.; Kinoshita, K.; Watanabe, T. Reliable wireless communications in battery management system of electric vehicles. In Proceedings of the 2017 Tenth International Conference on Mobile Computing and Ubiquitous Network (ICMU), Toyama, Japan, 3-5 October 2017; pp. 1-6. [CrossRef]

604. Faika, T.; Kim, T.; Khan, M. An Internet of Things (IoT)-Based Network for Dispersed and Decentralized Wireless Battery Management Systems. In Proceedings of the 2018 IEEE Transportation Electrification Conference and Expo (ITEC), Long Beach, CA, USA, 13-15 June 2018; pp. 1060-1064. [CrossRef]

605. Navitas Solutions Inc. Wireless Battery Management System: For Smart Grid and Electric Vehicle Applications; Navitas Solutions Inc.: Princeton, NJ, USA, 2016.

606. Zimmer, G. Wireless Battery Management Systems Highlight Industry's Drive for Higher Reliability; Linear Technology: Milpitas, CA, USA, 2007.

607. Analog Devices. Analog Devices Introduces Automotive Industry's First Wireless Battery Management System for Electric Vehicles: General Motors to Use ADI's Wireless Battery Management System Across its Ultium Battery Platform; Analog Devices: Norwood, MA, USA, 2020. 
608. Kleiner, J.; Heider, A.; Komsiyska, L.; Elger, G.; Endisch, C. Experimental Study on the Thermal Interactions in Novel Intelligent Lithium-Ion Modules for Electric Vehicles. In Proceedings of the 2021 IEEE ITherm The Intersociety Conference on Thermal and Thermomechanical Phenomena in Electronic Systems, San Diego, CA, USA, 1-4 June 2021.

609. Kleiner, J.; Heider, A.; Hanzl, C.; Komsiyska, L.; Elger, G.; Endisch, C. Thermal Behavior of an Intelligent Li-Ion Cell under Vehicle Conditions. In Proceedings of the IECON 2020-46th Annual Conference of the IEEE Industrial Electronics Society, Singapore, 18-21 October 2020; pp. 2081-2086. [CrossRef]

610. Chang, F.; Roemer, F.; Lienkamp, M. Influence of Current Ripples in Cascaded Multilevel Topologies on the Aging of Lithium Batteries. IEEE Trans. Power Electron. 2020, 35, 11879-11890. [CrossRef]

611. Uno, M.; Tanaka, K. Influence of High-Frequency Charge-Discharge Cycling Induced by Cell Voltage Equalizers on the Life Performance of Lithium-Ion Cells. IEEE Trans. Veh. Technol. 2011, 60, 1505-1515. [CrossRef]

612. Lewerenz, M.; Fuchs, G.; Becker, L.; Sauer, D.U. Irreversible calendar aging and quantification of the reversible capacity loss caused by anode overhang. J. Energy Storage 2018, 18, 149-159. [CrossRef]

613. Wong, D.; Shrestha, B.; Wetz, D.A.; Heinzel, J.M. Impact of high rate discharge on the aging of lithium nickel cobalt aluminum oxide batteries. J. Power Sources 2015, 280, 363-372. [CrossRef]

614. Soares, R.; Bessman, A.; Wallmark, O.; Lindbergh, G.; Svens, P. An Experimental Setup with Alternating Current Capability for Evaluating Large Lithium-Ion Battery Cells. Batteries 2018, 4, 38. [CrossRef]

615. Prasad, R.; Namuduri, C.; Kollmeyer, P. Onboard unidirectional automotive G2V battery charger using sine charging and its effect on li-ion batteries. In Proceedings of the 2015 IEEE Energy Conversion Congress and Exposition (ECCE), Montreal, QC, Canada, 20-24 September 2015; pp. 6299-6305. [CrossRef]

616. Korth Pereira Ferraz, P.; Kowal, J. A Comparative Study on the Influence of DC/DC-Converter Induced High Frequency Current Ripple on Lithium-Ion Batteries. Sustainability 2019, 11, 6050. [CrossRef] 